\title{
On Some Local Cohomology Spectral Sequences
}

\section{Josep Àlvarez Montaner ${ }^{1}$, Alberto F. Boix ${ }^{2, *}$, and Santiago Zarzuela ${ }^{3}$}

${ }^{1}$ Departament de Matemàtiques, Universitat Politècnica de Catalunya, Avinguda Diagonal 647, Barcelona 08028, Spain ${ }^{2}$ Department of Mathematics, Ben Gurion University of the Negev, P.O.B. 653 Beer-Sheva 8410501, Israel, and ${ }^{3}$ Departament de Matemàtiques i Informàtica, Universitat de Barcelona, Gran Via de les Corts Catalanes 585, Barcelona 08007, Spain

*Correspondence to be sent to: e-mail: albertof.boix@gmail.com

We introduce a formalism to produce several families of spectral sequences involving the derived functors of the limit and colimit functors over a finite partially ordered set. The 1st type of spectral sequences involves the left derived functors of the colimit of the direct system that we obtain by applying a family of functors to a single module. For the 2nd type we follow a completely different strategy as we start with the inverse system that we obtain by applying a covariant functor to an inverse system. The spectral sequences involve the right derived functors of the corresponding limit. We also have a version for contravariant functors. In all the introduced spectral sequences we provide sufficient conditions to ensure their degeneration at their 2nd page. As a consequence we obtain some decomposition theorems that greatly generalize the wellknown decomposition formula for local cohomology modules of Stanley-Reisner rings given by Hochster.

\section{Introduction}

Let $A$ be a commutative Noetherian ring, let $J \subseteq A$ be an ideal, and let $H_{J}^{r}(A)$ be the $r$ th local cohomology group of $A$ supported on $J$. When $A$ is $\mathbb{Z}^{n}$-graded (for some $n \in \mathbb{N}$ ) and 
$J$ is homogeneous, it is of interest to understand the graded pieces of $H_{J}^{r}(A)$ because of its connection with the cohomology of coherent sheaves of algebraic varieties given by the Deligne-Grothendieck-Serre correspondence [8, 20.3.16 (iv) and 20.4.4]. In case $A$ is a Stanley-Reisner ring with coefficients on a field $\mathbb{K}$, and $\mathfrak{m}$ is its irrelevant ideal, these graded pieces are encoded inside the celebrated Hochster's formula for Hilbert series of local cohomology modules $H_{\mathfrak{m}}^{r}(A)$ of Stanley-Reisner rings (see [59, Theorem II.4.1], [11, Theorem 5.3.8], and [41, Theorem 13.13]); it turns out that this decomposition preserves the grading of these local cohomology modules, by means of a formula proved by Gräbe [22, Theorem 2]. On the other hand, when $A$ is a polynomial ring with coefficients on a field $\mathbb{K}$, and $J$ is a squarefree monomial ideal, the graded pieces of $H_{J}^{r}(A)$ are explicitly described by means of results obtained by Mustaţă [42, Theorem 2.1 and Corollary 2.2] (see also [18]) and the corresponding formula for the Hilbert series was calculated by Terai [63] (see also [41, Corollary 13.16]); both decompositions preserve the grading, as proved by Mustaţă in [42]. It turns out that the results by Hochster (respectively, Terai) and Gräbe (respectively, Mustaţǎ) are equivalent, by means of the duality established by Miller [40, Corollary 6.7]. It is also worth noting that, in the last decade, several generalizations of Hochster's decomposition have appeared in the literature, being [62, Theorem 1], [19, Theorem 5.1], [10, Theorems 1.1 and 1.3], and [28, Theorem 4.5 and Lemma 4.6] the most relevant for the purposes of this paper (see also [38, Proposition 3.2 and Corollary 3.4], [41, Theorem 13.14], [53, Theorem 1.3], [50, Corollary 3], [2, Theorems 4.37 and 4.40], and [3, Theorems 4.24 and 4.26]).

Another different approach was adopted by Àlvarez Montaner et al. in [5]; indeed, building upon a Mayer-Vietoris spectral sequence

$$
E_{2}^{-i, j}=\mathbb{L}_{i} \operatorname{colim}_{p \in P} H_{I_{p}}^{j}(A) \Longrightarrow H_{I}^{j-i}(A),
$$

(where $A$ is a polynomial ring with coefficients on a field, $I$ is the defining ideal of an arrangement of linear varieties, $I=I_{1} \cap \ldots \cap I_{n}$ is a primary decomposition, and $P$ is the partially ordered set made up by all the possible different sums of the ideals $I_{1}, \ldots, I_{n}$ ordered by reverse inclusion, so each element $p \in P$ is identified with one of these sums, which one denotes by $I_{p}$ ) they show that, under these assumptions, the spectral sequence (1) degenerates at its 2nd page. Along the way, they provide [5, Corollary 1.3] a closed formula for topological Betti numbers of complements of arrangements of linear varieties, which may be regarded as a local cohomology interpretation of the celebrated Goresky-MacPherson formula [20, III.1.3.Theorem A]. Finally, by studying the extension problems of the filtration produced by this degeneration when $I$ is a 
squarefree monomial ideal, they show that these extensions are, in general, nontrivial, and are determined by the $\mathbb{Z}^{n}$-graded structure of $H_{I}^{j}(A)$.

Assume for a while that $\mathbb{K}$ is any field, $A=\mathbb{K} \llbracket x_{1}, \ldots, x_{n} \rrbracket, \mathfrak{m}=\left(x_{1}, \ldots, x_{n}\right)$, and $J \subseteq A$ is any ideal; one might ask about the existence of a spectral sequence

$$
E_{2}^{i, j}=\mathbb{R}^{i} \lim _{p \in P} H_{\mathfrak{m}}^{j}\left(A / I_{p}\right) \quad \Longrightarrow H_{\mathfrak{m}}^{i+j}(A / I)
$$

Unfortunately, such a spectral sequence cannot exist; indeed, otherwise it would collapse by a result of Jensen [30, 7.1], and therefore it would produce a decomposition of $H_{\mathfrak{m}}^{r}(A / I)$ that would contradict Hochster's one. It is worth noting that, under the additional assumption that $\mathbb{K}$ has prime characteristic, Lyubeznik introduced in [36, Section 4] a functor $\mathcal{H}:=\mathcal{H}_{A, A / J}$ that has the following behavior with respect to local cohomology modules:

$$
\mathcal{H}\left(H_{\mathfrak{m}}^{n-r}(A / J)\right) \cong H_{J}^{r}(A),
$$

so it was natural to ask about the existence of a spectral sequence that would correspond to (1) under $\mathcal{H}$.

The 1st goal of this paper is to build a formalism to construct several spectral sequences that, on the one hand, recover and extend the Mayer-Vietoris spectral sequence (1) (see also [37, Theorem 2.1]) for other types of functors and, on the other hand, to fix and generalize the false spectral sequence (2). Broadly speaking, we produce two types of spectral sequences; the 1st ones are associated to a single module; given this module, one applies a family of functors that produce a direct system. Since the 2nd page of these spectral sequences involves the left derived functors of the colimit, we will refer to them as homological spectral sequences. In contrast, the 2nd type of spectral sequences we produce is attached to an inverse system of modules; indeed, given such an inverse system, we apply a single functor to produce another inverse system. Since the 2nd page of these spectral sequences involves the right derived functors of the (inverse) limit, we refer to them as cohomological spectral sequences.

The 2nd goal is to study the degeneration of these spectral sequences at their corresponding 2nd pages and the filtration that this degeneration provides. From this filtration we get a collection of short exact sequences for which we study its extension problems. As a consequence of this study we produce some general Hochster-type decompositions not only for local cohomology modules but also for more general functors. 
The organization of this article is as follows. Since all the spectral sequences that appear in this manuscript involve the left (respectively, the right) derived functors of the colimit (respectively, limit) functors, we review in Section 2 the facts we need later on about the categories of direct and inverse systems over finite partially ordered sets; albeit most of the material presented in Section 2 is known, we present, as far as possible, a self-contained treatment for the convenience of the reader. The derived functors of the colimit and limit can be described by means of the Roos complexes that we recall in Section 3.

In Section 4 we introduce our 1st family of homological spectral sequences. Given an $A$-module $M$ and a set of functors satisfying some technical conditions (see Setup 4.3) we construct a direct system $\left\{T_{p}(M)\right\}_{p \in P}$ over a finite poset $P$ with a distinguished final element $T(M)$. The following generalization of the Mayer-Vietoris spectral sequence of local cohomology modules given in [4] and [37] is the main result of this section.

Theorem 4.6. Under the previous assumptions we have the following spectral sequence:

$$
E_{2}^{-i, j}=\mathbb{L}_{i} \operatorname{colim}_{p \in P} \mathbb{R}^{j} T_{[*]}(M) \Longrightarrow \mathbb{R}^{j-i} T(M)
$$

This is a spectral sequence in the category of $A$-modules but it can be enlarged with an extra structure as graded modules, $D$-modules, $F$-modules, or modules over the group ring (see Theorem 4.8 and the examples listed afterwards). Several examples are shown in Section 4.2 including generalized local cohomology modules, ideal transforms, and local cohomology associated to pairs of ideals. In particular, this is the 1st time, to the best of our knowledge, that a Mayer-Vietoris sequence for this later example appears in the literature. We point out that the technical conditions imposed on the functors are essential in the construction of the spectral sequence and, for example, it does not apply to the contravariant Hom functor.

Moreover, in the spirit of [4] we provide sufficient conditions to guarantee the degeneration of the spectral sequences at their corresponding 2nd page (see Theorem 4.11). The associated filtration of $\mathbb{R}^{r} T(M)$ that we obtain is described in Corollary 4.12, as well as an enriched version (see Corollary 4.13). In Section 4.5 we use this filtration to recover and extend the formula for the characteristic cycles of local cohomology modules obtained in [4]; we also produce a closed formula for certain generalized Lyubeznik numbers introduced in [49] and we provide a closed formula for the length of local cohomology modules in the category of Lyubeznik's F-modules [36]. 
In Section 5 we introduce our 2nd family of spectral sequences. The setup is completely different from the previous one as we start with an inverse system $V:=$ $\left\{V_{p}\right\}_{p \in P}$ over a finite poset $P$ and a single covariant functor $T$ satisfying some technical conditions (see Setup 5.4). Then we produce an inverse system $\mathcal{T}:=\left\{T\left(V_{p}\right)\right\}_{p \in P}$. The following result has to be understood as a generalization of the long exact sequence of local cohomology modules.

Theorem 5.6. Under the previous assumptions we have the following spectral sequence:

$$
E_{2}^{i, j}=\mathbb{R}^{i} \lim _{p \in P} \mathbb{R}^{j} \mathcal{T}(V) \Longrightarrow \mathbb{R}^{i+j}\left(\lim _{p \in P} \circ \mathcal{T}\right)(V) .
$$

If, in addition, there is a natural equivalence of functors $\lim _{p \in P} \circ \mathcal{T} \cong T \circ \lim _{p \in P}$, and $V$ is acyclic with respect to the limit, then the previous spectral sequence can be arranged in the more accessible manner:

$$
E_{2}^{i, j}=\mathbb{R}^{i} \lim _{p \in P} \mathbb{R}^{j} \mathcal{T}(V) \Longrightarrow \mathbb{R}^{i+j} T\left(\lim _{p \in P} V_{p}\right)
$$

In this case we may also associate an extra structure to this spectral sequence (see Theorem 5.17). The examples that we present in Section 5.2 include the covariant Hom, generalized local cohomology, generalized ideal transform local cohomology with respect to pairs of ideals, and inverse systems of ideals. Moreover, changing our initial poset, we may consider local cohomology of toric face rings. In Theorem 5.18 we also provide sufficient conditions to guarantee the degeneration at the 2nd page in this case.

In Section 6 we play a similar game with an inverse system $V:=\left\{V_{p}\right\}_{p \in P}$ over a finite poset $P$ and a single contravariant functor $T$. In this way we produce a direct system $\mathcal{T}:=\left\{T\left(V_{p}\right)\right\}_{p \in P}$ and a spectral sequence involving the derived functors of the colimit (see Theorem 6.3). The main example for this case is the contravariant Hom.

In Section 7 we provide some decomposition theorems that follow naturally in the case that the spectral sequences introduced in the previous sections degenerate at the 2nd page. For homological spectral sequences that were constructed along Section 4 we obtain the following result.

Theorem 7.5. There is a $\mathbb{K}$-vector space isomorphism

$$
\mathbb{R}^{j} T(M) \cong \bigoplus_{q \in P} \mathbb{R}^{h_{q}} T_{q}(M)^{\oplus m_{j, q}}
$$

where $h_{q}$ is the single value where $\mathbb{R}^{j} T_{q}(M)$ does not vanish and $m_{j, q}=\operatorname{dim}_{\mathbb{K}} \widetilde{H}_{h_{q}-j-1}$ $\left(\left(q, 1_{\widehat{P}}\right) ; \mathbb{K}\right)$ with $\left(q, 1_{\widehat{P}}\right)$ being the order complexes associated to elements of the poset. 
Indeed, this decomposition preserves the grading as shown in Theorem 7.8. In the case that $A=\mathbb{K}\left[x_{1}, \cdots, x_{n}\right]$ is a polynomial ring, $T=\Gamma_{I}$, and $I$ is a monomial ideal we recover the formula given by Mustaţă and Terai. We also want to single out here that this result produces, to the best of our knowledge, a new decomposition (see Theorem 7.14) for the defining ideal of Stanley toric face rings over a field of prime characteristic.

In the case of cohomological spectral sequences (see Theorem 7.2 for the case of homological spectral sequences associated to an inverse system, including a decomposition for the so-called deficiency modules) that were constructed in Section 5, we obtain a Hochster-type decomposition.

Theorem 7.1.There is a $\mathbb{K}$-vector space isomorphism

$$
\mathbb{R}^{j} T\left(\lim _{p \in P} V_{p}\right) \cong \bigoplus_{q \in P} \mathbb{R}^{d_{q}} T\left(V_{q}\right)^{\oplus M_{j, q}}
$$

where $d_{q}$ is the single value where $\mathbb{R}^{j} T\left(V_{p}\right)$ does not vanish and $M_{j, q}=\operatorname{dim}_{\mathbb{K}} \widetilde{H}^{j-d_{q}-1}$ $\left(\left(q, 1_{\widehat{P}}\right) ; \mathbb{K}\right)$.

This isomorphism preserves the grading as well by Theorem 7.7. When $V=\left\{A / I_{p}\right\}$ is the inverse system associated to a Stanley-Reisner ring $A / I$ and $T=\Gamma_{\mathfrak{m}}$ we recover Hochster's formula; more generally, when $V=\left\{A / I_{p}\right\}$ is the inverse system associated to $A / I$, where $I$ is any monomial ideal we recover Takayama's formula as formulated by Brun and Römer in [10, Corollary 2.3]. In addition, this decomposition also recovers the Brun-Bruns-Römer decomposition [9, Theorem 1.3] for the so-called Stanley toric face rings (see Theorem 7.9). We point out that our result produces, to the best of our knowledge, a new decomposition for local cohomology modules of certain central arrangements of linear varieties over a field (see Theorem 7.12). The issue in this case is that, in general, we do not have an isomorphism $\lim _{p \in P} A / I_{p} \cong$ $A / I$ (see Remarks 5.2 and 5.3). Whenever the isomorphism holds we produce a closed formula for the Castelnuovo-Mumford regularity of these arrangements (see Theorem 7.18); as an immediate consequence, we obtain an alternative proof, specific for this kind of arrangements, of the so-called Subspace Arrangements Theorem (see Remark 7.19), originally proved by Derksen and Sidman [15, Theorem 2.1] for arbitrary central arrangements.

When $A$ contains a field of positive characteristic, Enescu and Hochster show in [19, Theorem 5.1] that Hochster's decomposition of Stanley-Reisner rings is compatible 
with the natural Frobenius action on the local cohomology modules, obtaining in particular that local cohomology modules of Stanley-Reisner rings have only a finite number of F-stable submodules, that is, $A$-submodules $N \subseteq H_{\mathfrak{m}}^{r}(A)$ such that the action of Frobenius maps $N$ into itself. The interest for studying these F-stable submodules arises naturally in the study of singularities of algebraic varieties in prime characteristic, due to its connection with test ideals. In Theorem 7.20, we show that Brun-Bruns-Römer decomposition of Stanley toric face rings of prime characteristic is compatible with this natural Frobenius action, and therefore they only have a finite number of F-stable submodules; in particular, we recover and extend [19, Theorem 5.1], because Stanley-Reisner rings are a particular case of Stanley toric face rings [46, Example 2.2 (i)].

In Section 8, following the spirit of [5, Section 3], we study the extension problems attached to the corresponding filtrations produced by the degeneration of our spectral sequences. Our focus is on the cohomological spectral sequence associated to $T=\Gamma_{\mathfrak{m}}$. The main result is Theorem 8.13 that may be regarded as a Gräbe's type description formula.

\section{The Categories of Inverse and Direct Systems}

The purpose of this section is to review the facts we shall need later on about posets and the categories of inverse and direct systems; we try to present, as far as possible, a self-contained exposition of this topic for the reader's profit.

Let $\left(P_{1} \leq\right)$ be a partially ordered set (from now on, poset for the sake of brevity). We shall regard $P$ as a small category that has as objects the elements of $P$ and, given $p, q \in P$, there is one morphism $p \rightarrow q$ if $p \leq q$. If $P$ contains a unique minimal (respectively, maximal) element then this is called the initial (respectively, terminal) element of $P$ and it will be denoted by $0_{P}$ (respectively, $1_{P}$ ). Adding an initial and a terminal element to $P$ (even in case $P$ have them) we may consider the poset $(\widehat{P}, \leq)$, where $\widehat{P}:=P \cup\left\{0_{\widehat{P}}, 1_{\widehat{P}}\right\}$.

Throughout this paper, we mainly consider the following examples of posets.

Example 2.1. Let $A$ be a commutative Noetherian ring, let $I \subseteq A$ be an ideal such that $I=I_{1} \cap \ldots \cap I_{n}$ for certain ideals $I_{1}, \ldots, I_{n}$ of $A$. In this case, we can produce a poset $P$ in the following way: $P$ will be the poset given by all the possible different sums of the ideals $I_{1}, \ldots, I_{n}$ ordered by reverse inclusion. So, any element $p \in P$ is identified with a certain sum of the ideals $I_{1}, \ldots, I_{n}$, which we denote by $I_{p}$. 
Example 2.2. Given $\Sigma \subseteq \mathbb{R}^{d}$ a rational pointed fan [46, p. 538], we define $P$ as the poset given by all the possible faces of $\Sigma$ ordered by inclusion; in other words, we identify each face $C \subseteq \Sigma$ with a point $p_{C} \in P$. Moreover, given faces $C \subseteq C^{\prime}$, one has $p_{C} \leq p_{C^{\prime}}$

Example 2.3. Let $\Delta$ be a simplicial complex. In this case, we define $P$ as the poset made up by the faces of $\Delta$ ordered by inclusion; indeed, we identify a face $F \subseteq \Delta$ with a point $p_{F} \in P$, and given faces $F \subseteq F^{\prime}$ we have $p_{F} \leq p_{F^{\prime}}$.

Notice that in all the above examples, the poset $P$ is finite; this will be an assumption that we preserve during the rest of this paper. Indeed, for this reason we want to single out the following:

Assumption 2.4. Hereafter, we shall always assume that $P$ is a finite poset.

\subsection{The categories of inverse and direct systems and some equivalences}

Throughout this paper, let $\mathcal{A}$ be the category of $A$-modules, where $A$ denotes a commutative Noetherian ring:

(a) A direct system over $P$ valued on $\mathcal{A}$ is a covariant functor $P \stackrel{F}{\longrightarrow} \mathcal{A}$; in what follows, we will denote either by $F_{p}$ or $F(p)$ the value of $F$ at $p \in P$.

(b) An inverse system over $P$ valued on $\mathcal{A}$ is a contravariant functor $P \stackrel{G}{\longrightarrow} \mathcal{A}$; hereafter, we will denote either by $G_{p}$ or $G(p)$ the value of $G$ at $p \in P$.

From now on, $\operatorname{Dir}(P, \mathcal{A})$ (respectively, $\operatorname{Inv}(P, \mathcal{A})$ ) will denote the category of direct (respectively, inverse) systems valued on $\mathcal{A}$; it is known that both are abelian categories $[56,5.94]$.

Before going on, we would like to exhibit some examples of direct and inverse systems for the convenience of the reader; in order to do so, we need to review the following:

Definition 2.5 (Hasse, Voght). $\quad$ Let $P$ be a finite poset. The Hasse-Voght diagram of $P$ is obtained by drawing the elements of $P$ as dots, with $x$ drawn lower than $y$ if $x<y$, and with an edge between $x$ and $y$ whenever $y$ covers $x$, that is, if $x<y$ and no $z \in P$ satisfies $x<z<y$.

Now, we are ready for our examples. 
Example 2.6. We go back to Example 2.1; in the below picture, we take $n=2$, and we draw on the left the poset $P$ and on the right the direct system $F$ given by $F(p)=\Gamma_{I_{p}}(M)$, where $M$ is any $A$-module. Notice that, in all the following pictures, the non-dotted lines and arrows are edges of the poset $P$, and the dotted ones are edges of the poset $\widehat{P}$.
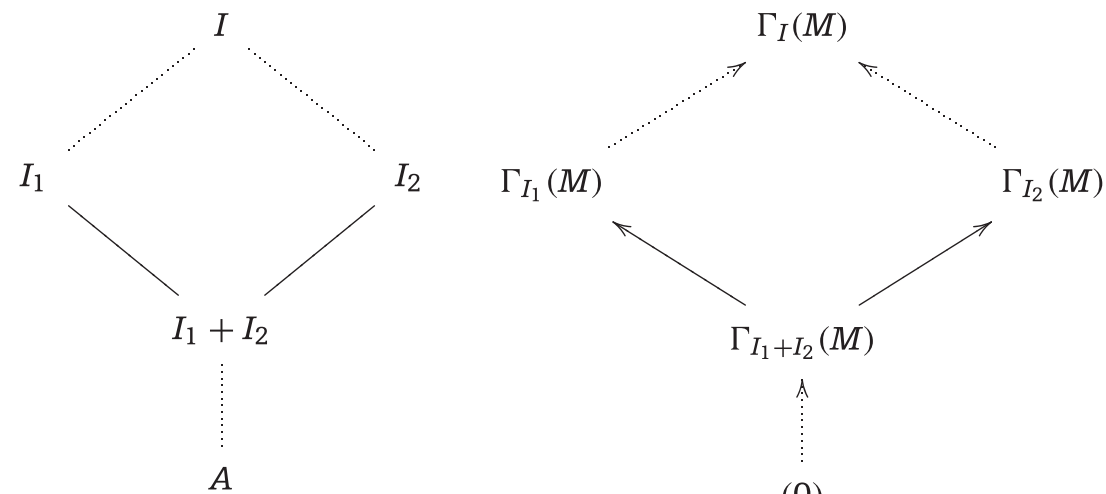

Again in Example 2.1, we take $n=2$, but now on the right we draw the inverse system $G$ given by the assignment $G(p)=M / I_{p} M$.

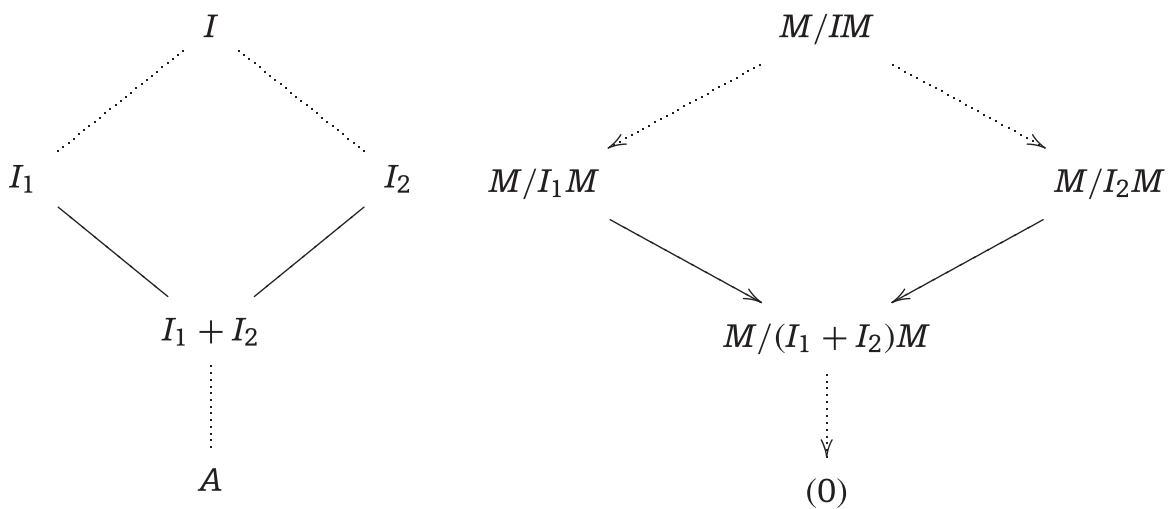

From now onward, we omit the initial element of the poset $\widehat{P}$ in our Hasse-Voght diagrams. Now, we want to illustrate Example 2.1 in case $n=3$ in a couple of specific situations; indeed, take the ring $A=\mathbb{K}\left[x_{1}, \ldots, x_{6}\right]$, where $\mathbb{K}$ is any field. Firstly, 
if $I=\left(x_{1}, x_{2}\right) \cap\left(x_{3}, x_{4}\right) \cap\left(x_{5}, x_{6}\right)$ then our poset can be drawn in the following way:

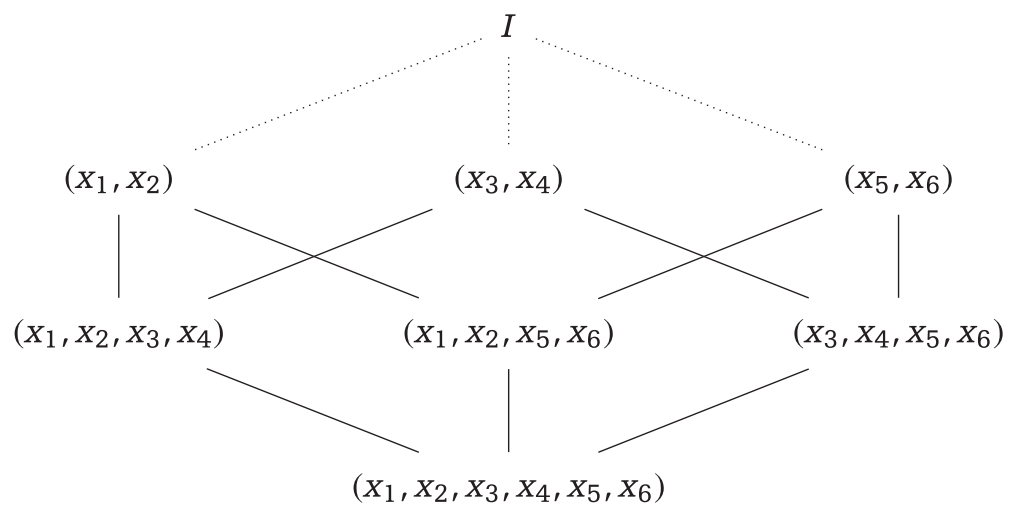

In the case that $I=\left(x_{1}, x_{2}\right) \cap\left(x_{1}, x_{3}\right) \cap\left(x_{2}, x_{3}\right)$ our picture becomes more simple because we identify these sums that describe the same ideal of $A$ :

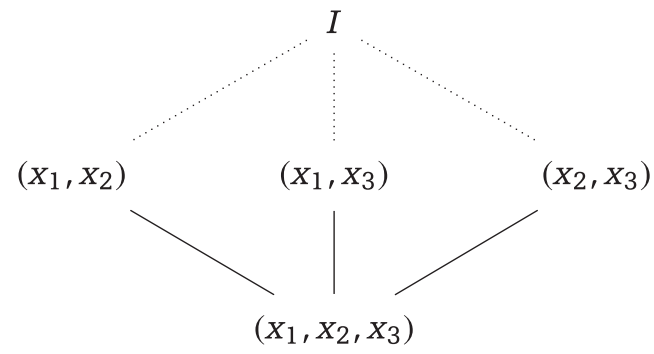

\subsection{Equivalent approaches}

We decided to choose this approach to study derived functors of limits and colimits. Nevertheless, there are other equivalent approaches to this subject. Some of them are reviewed in what follows. The reader is encouraged to follow his/her own preferences.

\subsubsection{The category of sheafs on posets}

Given a poset $P$ and given $p, q \in P$, the closed interval of elements between $p$ and $q$ will be denoted by $[p, q]:=\{z \in P \mid \quad p \leq z \leq q\}$ and forms a subposet of $P$. In a similar way, we can also construct the intervals $(p, q],[p, q)$, and $(p, q)$.

The Alexandrov topology on $P$ is the topology where the open sets are the subsets $U$ of $P$ such that $p \in U$ and $p \leq q$ implies $q \in U$. In fact, this is the unique topology that one can attach to $P$ verifying this property. Moreover, the subsets of the form $\left[p, 1_{\widehat{P}}\right.$ ) form an open basis for this topology. On the other hand, the dual Alexandrov topology on $P$ is the topology where the open sets are the subsets $U$ of $P$ such that $p \in U$ 
and $q \leq p$ implies $q \in U$. Once more, this is the unique topology that one can attach to $P$ verifying this property and the subsets of the form $\left(0_{\widehat{P}}, p\right]$ form an open basis for this topology. We underline that this can be viewed as the Alexandrov topology on the opposite poset $P^{o p}=(P, \preceq)$, where $p \preceq q$ if and only if $q \leq p$.

Example 2.7. Given $X$ a set with $X=X_{1} \cup \ldots \cup X_{n}$ for certain subsets $X_{j} \subseteq X$, we define $P$ as the poset given by all the possible different intersections of $X_{1}, \ldots, X_{n}$ ordered by inclusion. Thus, any element $p \in P$ is identified with a certain intersection of the $X_{j} \mathrm{~s}$, which we denote by $X_{p}$. The reader will easily note that this example recovers and extends Example 2.1.

We shall fix some additional notation before going on.

Notation 2.8. In the sequel, we shall denote by $\operatorname{Sh}(P, \mathcal{A})$ (respectively, $\left.\operatorname{Sh}\left(P^{o p}, \mathcal{A}\right)\right)$ the category of sheaves on $P$ (respectively, $P^{o p}$ ) valued on $\mathcal{A}$.

We conclude this part with the following elementary statement, which will be useful in what follows; we omit the details.

Lemma 2.9. Let $(P, \leq)$ be a poset regarded as a topological space with the Alexandrov topology. Then, for any $p \in P$, the basic open set $\left[p, 1_{\widehat{P}}\right]$ is contractible.

We also want to establish now the analogous of Lemma 2.9 regarding $P$ as a topological space with the dual Alexandrov topology; once again, we skip the details.

Lemma 2.10. Let $(P, \leq)$ be a poset regarded as a topological space with the dual Alexandrov topology. Then, for any $p \in P$, the basic open set $\left(0_{\widehat{P}}, p\right.$ ] is contractible.

\subsubsection{The category of AP-modules}

The purpose of this part is to review the category of left $A P$-modules, as presented in [9, Section 6]; in fact, whereas in op. cit. the authors established this notion in the context of inverse systems, here our definition deals with direct systems. However, it is clear that both notions can be mutually recovered just by taking the opposite order on the poset $P$.

Definition 2.11. A left $A P$-module $M$ is a system $\left(M_{p}\right)_{p \in P}$ of left $A$-modules and, for $p \leq q$, homomorphisms $M_{p} \stackrel{M_{p q}}{\longrightarrow} M_{q}$ with the property that, for all $p \leq q \leq z, M_{p p}=\mathbb{1}_{M_{p}}$ 
and $M_{p q} \circ M_{q z}=M_{p z}$; moreover, a homomorphism $M \stackrel{f}{\longrightarrow} N$ of left $A P$-modules consists of, for $p \in P$, homomorphisms $M_{p} \stackrel{f_{p}}{\longrightarrow} N_{p}$ of left $A$-modules such that, for any $p \leq q$ in $P$, the following square commutes.

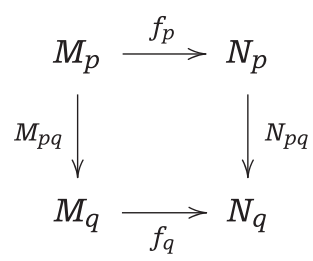

We shall denote the group of homomorphisms from $M$ to $N$ by $\operatorname{Hom}_{A P}(M, N)$. Moreover, we shall denote by $A P$ - Mod the category of left $A P$-modules.

In a similar way, just reversing the convenient morphisms, we can also construct the category $A P^{o p}-$ Mod of left $A P^{o p}$-modules.

\subsubsection{Modules over the incidence algebra}

Recall that $P$ is a finite poset (see Assumption 2.4) and that $A$ is a commutative ring.

Definition 2.12 (Incidence algebra). We define the incidence algebra $I(P, A)$ of $P$ over $A$ as follows: $I(P, A)$ is the $A$-algebra with underlying $A$-module

$$
I(P, A):=\bigoplus_{p \leq q} A \cdot \mathbf{e}_{p \leq q}
$$

endowed with the following multiplication rule:

$$
\left(\mathbf{e}_{p \leq q}\right) \cdot\left(\mathbf{e}_{p^{\prime} \leq q^{\prime}}\right):=\left\{\begin{array}{l}
\mathbf{e}_{p \leq q^{\prime}}, \text { if } q=p^{\prime} \\
0, \text { otherwise }
\end{array}\right.
$$

Notice that the elements $\mathbf{e}_{p \leq p}$ are idempotent in $I(P, A)$ and that, since $P$ is finite, the element $\sum_{p \in P} \mathbf{e}_{p \leq p}$ is the multiplicative unit in $I(P, A)$.

\subsubsection{The four previous categories are equivalent}

In this part, we establish the fact that all the foregoing approaches are equivalent; the statement is as follows: 
Proposition 2.13. The following categories are equivalent:

(a) $\operatorname{Dir}(P, \mathcal{A})$, the category of direct systems on $P$ valued in $\mathcal{A}$.

(b) $\operatorname{Sh}(P, \mathcal{A})$, the category of sheaves on $P$ valued in $\mathcal{A}$ regarding $P$ as a topological space with the Alexandrov topology.

(c) $A P$ - Mod, the category of left $A P$-modules.

(d) The category of left modules over the incidence algebra $I\left(P^{o p}, A\right)$. On the other hand, the below four categories are equivalent:

(i) $\operatorname{Inv}(P, \mathcal{A})$, the category of inverse systems on $P$ valued in $\mathcal{A}$.

(ii) $\operatorname{Sh}\left(P^{o p}, \mathcal{A}\right)$, the category of sheaves on $P^{o p}$ valued in $\mathcal{A}$ regarding $P$ as a topological space with the Alexandrov topology.

(iii) $A P^{o p}$ - Mod, the category of left $A P^{o p}$-modules.

(iv) The category of left modules over the incidence algebra $I(P, A)$.

Sketch of proof. A detailed proof of the equivalence between categories (i)-(iv) can be found in [9, 6.5 and 6.6]; the equivalence between categories (a)-(d) can be proved in a similar way. What we want to do here is to review how to construct the equivalence between $\operatorname{Dir}(P, \mathcal{A})$ and $\operatorname{Sh}(P, \mathcal{A})$, the category of sheaves on $P$ valued in $\mathcal{A}$ regarding $P$ as a topological space with the Alexandrov topology, because we will use this equivalence several times throughout this paper.

On the one hand, given an object $\mathcal{F} \in \operatorname{Sh}(P, \mathcal{A})$, one can cook up a direct system $\Gamma(P, \mathcal{F})$ in the following way: for each $p \in P$, set $\Gamma(P, \mathcal{F})_{p}:=\mathcal{F}\left(\left[p, 1_{\widehat{P}}\right)\right)$. Moreover, for any $p \leq q$ the homomorphism $\Gamma(P, \mathcal{F})_{p} \longrightarrow \Gamma(P, \mathcal{F})_{q}$ equals the restriction map induced by the inclusion of open sets $\left[q, 1_{\widehat{P}}\right) \subseteq\left[p, 1_{\widehat{P}}\right)$. On the other hand, given a direct system $M$ we can produce a presheaf $\widetilde{M}$ as follows: for each open set $U \subseteq P$ set $\widetilde{M}(U):=\operatorname{colim}_{p \in U} M_{p}$. In addition, given open sets $V \subseteq U \subseteq P$, the map $\widetilde{M}(U) \longrightarrow \widetilde{M}(V)$ is just the natural restriction of colimits. Finally, since $\left[p, 1_{\widehat{P}}\right.$ ) is contained in any open neighborhood of $P$ (this is by definition of the Alexandrov topology), the presheaf $\widetilde{M}$ is isomorphic to the associated sheaf of $A$-modules on $P$; in this way, the symbol $\widetilde{(-)}$ defines a well-defined functor from $\operatorname{Dir}(P, \mathcal{A})$ to $\operatorname{Sh}(P, \mathcal{A})$.

\subsection{Injective, projective, and flasque inverse systems}

The goal of this subsection is to review, not only the well-known fact that the category $\operatorname{Inv}(P, \mathcal{A})$ has enough injective and projective objects [47, Satz 1 and Satz 2], but also to describe explicitly certain classes of injective (respectively, projective) objects that will play a key role later on. 
We start with the case of injective inverse systems, as described in the following result; the interested reader may like to consult either [47, Satz 1 and Beweis] or [45, Lemma A.4.3 and Remark A.4.4] for details. The reader will easily note that, since we are working with finite posets, in the below result we only need to deal with direct sums instead of direct products; namely the following:

Theorem 2.14. Any injective object of $\operatorname{Inv}(P, \mathcal{A})$ is a direct summand of a direct sum of injectives of the form $E_{\geq q}$ for some $q \in P$, where $E=E_{A}(A / \mathfrak{p})$ is an indecomposable injective $A$-module, and

$$
\left(E_{\geq q}\right)_{p}:=\left\{\begin{array}{l}
E, \text { if } p \in\left[q, 1_{\widehat{P}}\right) \\
0, \text { otherwise. }
\end{array}\right.
$$

Here, given $p \leq r$ the map $\left(E_{\geq q}\right)_{r} \longrightarrow\left(E_{\geq q}\right)_{p}$ is zero if either source or target is zero, otherwise it equals the identity on $E$. In addition, any inverse system $G$ admits an injective (not necessarily minimal) resolution $0 \longrightarrow G \longrightarrow E^{0} \longrightarrow E^{1} \longrightarrow \ldots$, such that, for any $i \in \mathbb{N}, E^{i}$ can be expressed as a direct sum of injectives of the form $E_{\geq q}$.

Remark 2.15. The statement of Theorem 2.14 is not fully satisfactory in the sense that one would wish to have a structure theorem of injectives $\operatorname{in} \operatorname{Inv}(P, \mathcal{A})$; in other words, we would like to have a description of indecomposable injective objects $\operatorname{in} \operatorname{Inv}(P, \mathcal{A})$. We do not know whether such description exists.

We also want to single out the multigraded version of Theorem 2.14, because it will be useful later on; notice that [47, Satz 1 and Beweis] also works in this multigraded setting because, in particular, there are enough multigraded (a.k.a. ${ }^{*}$ ) injectives objects, and these *injectives satisfy an analogous Matlis-type structure theorem. The interested reader may like to consult [8, Chapter 13] and the references given therein for additional information.

Theorem 2.16. Let $* \mathcal{A}$ be the category of $\mathbb{Z}^{n}$-graded $A$-modules, where $A$ is a commutative Noetherian $\mathbb{Z}^{n}$-graded ring (for some $n \geq 1$ ). Then, any ${ }^{*}$ injective object of $\operatorname{Inv}\left(P,{ }^{*} \mathcal{A}\right)$ is a direct summand of a direct sum of ${ }^{*}$ injectives of the form ${ }^{*} E_{\geq q}$ for some $q \in P$, where ${ }^{*} E={ }^{*} E_{A}(A / \mathfrak{p})$ is an indecomposable ${ }^{*}$ injective $A$-module, and

$$
\left({ }^{*} E_{\geq q}\right)_{p}:=\left\{\begin{array}{l}
{ }^{*} E, \text { if } p \in[q, 1 \widehat{P}) \\
0, \text { otherwise. }
\end{array}\right.
$$


Here, given $p \leq r$ the map $\left({ }^{*} E_{\geq q}\right)_{r} \longrightarrow\left({ }^{*} E_{\geq q}\right)_{p}$ is zero if either source or target is zero, otherwise it equals the identity on ${ }^{*} E$. In addition, any inverse system $G$ admits an *injective (not necessarily minimal) resolution $0 \longrightarrow G \longrightarrow{ }^{*} E^{0} \longrightarrow{ }^{*} E^{1} \longrightarrow \ldots$, such that, for any $i \in \mathbb{N},{ }^{*} E^{i}$ can be expressed as direct sum of $*$ injectives of the form $* E_{\geq q}$.

Now, the case of projective objects; we refer to [47, Satz 2 and Beweis] for details.

Theorem 2.17. Given a projective $A$-module $F$ and given $p \in P$, the inverse system $F_{\leq p}$ defined by

$$
\left(F_{\leq p}\right)_{q}:=\left\{\begin{array}{l}
F, \text { if } q \in\left(0_{\widehat{P}}, p\right] \\
0, \text { otherwise }
\end{array}\right.
$$

is a projective object of $\operatorname{Inv}(P, \mathcal{A})$; in particular, $A_{\leq p}$ is so. Here, given $q \leq r$ the homomorphism $\left(F_{\leq p}\right)_{r} \longrightarrow\left(F_{\leq p}\right)_{q}$ is zero if either source or target is zero, otherwise it equals the identity on $F$. Furthermore, any inverse system $G$ admits a projective (not necessarily minimal) resolution

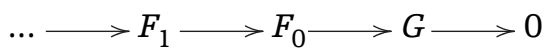

such that, for any $i \in \mathbb{N}, F_{i}$ can be expressed as a direct sum of projectives of the form $A_{\leq p}$.

Since the category of inverse systems has enough injectives [45, Lemma A.4.3], one has that the right derived functors of the limit can be defined in the usual way through injective resolutions [45, Corollary A.4.5]; however, in this case, it turns out that one needs to compute explicitly these derived functors and, with this purpose in mind, we have to restrict our attention to the following subclass of objects of $\operatorname{Inv}(P, \mathcal{A})$.

Definition 2.18. We say that an object $G$ of $\operatorname{Inv}(P, \mathcal{A})$ is flasque if, for any open set $U \subseteq P$ (regarding $P$ as a topological space with the dual Alexandrov topology), the natural restriction map $\lim _{p \in P} G(p) \longrightarrow \lim _{u \in U} G(u)$ is surjective.

Now, we want to single out the following result because it will play some role in Proposition 2.20. Its proof follows from the fact that any retract of a surjection is also a surjection, and it will be omitted. 
Lemma 2.19. Any direct summand of a flasque inverse system is also flasque.

Flasque inverse systems are useful for our purposes because of the following:

Proposition 2.20. The following statements hold.

(i) Any inverse system can be embedded into a flasque one; whence $\operatorname{Inv}(P, \mathcal{A})$ has enough flasque objects.

(ii) Any injective inverse system is flasque.

(iii) Any inverse system admits a flasque resolution.

(iv) The right derived functors of the limit can be computed through a flasque resolution.

Proof. Let $G$ be an object of $\operatorname{Inv}(P, \mathcal{A})$ and fix $p \in P$. We consider the following inverse system:

$$
\Pi^{0}(p):=\Pi^{0}(G)(p):=\prod_{p_{0} \leq p} G\left(p_{0}\right)
$$

Moreover, given $p \leq q$ we set $\Pi^{0}(q) \longrightarrow \Pi^{0}(p)$ as the natural projection. In this way, $\Pi^{0}(G)$ defines an inverse system that we claim is flasque.

Before showing so, we check that, for any open subset $W$ of $P^{o p}$, one has that

$$
\lim _{w \in W} \Pi^{0}(w) \cong \prod_{w \in W} G(w)
$$

Indeed, given $w_{0} \in W$, consider the natural projection $\prod_{w \in W} G(w) \stackrel{\pi_{w_{0}}}{\longrightarrow} \prod_{w \leq w_{0}} G(w)$; on the other hand, we also consider the natural restriction map

$$
\lim _{w \in W} \Pi^{0}(w) \stackrel{p_{w_{0}}}{\longrightarrow} \Pi^{0}\left(w_{0}\right)
$$

Thus, for any $x_{0} \leq w_{0}$, if $\Pi^{0}\left(w_{0}\right) \stackrel{p_{w_{0} x_{0}}}{\longrightarrow} \Pi^{0}\left(x_{0}\right)$ denotes the corresponding structural map in the inverse system $\Pi^{0}$, then one has the following commutative triangle:

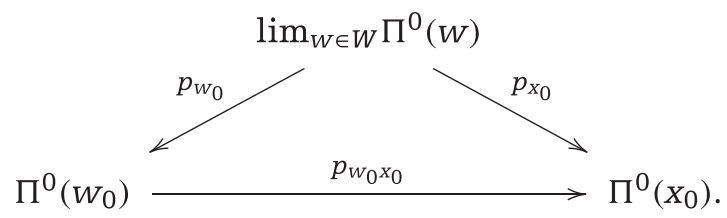


In this way, the universal property of the limit guarantees the existence of a unique homomorphism of $A$-modules

$$
\prod_{W \in W} G(w) \stackrel{\varphi=\varphi_{W}}{\longrightarrow} \lim _{W \in W} \Pi^{0}(w)
$$

such that $p_{w_{0}} \varphi=\pi_{w_{0}}$; on the other hand, the universal property of the direct product ensures the existence of a unique homomorphism of $A$-modules

$$
\lim _{w \in W} \Pi^{0}(w) \stackrel{\psi=\psi_{W}}{\longrightarrow} \prod_{w \in W} G(w)
$$

such that $\pi_{w_{0}} \psi=p_{w_{0}}$. We claim that $\psi$ and $\varphi$ are mutually inverses; indeed, we only have to point out that

$$
\pi_{w_{0}}(\psi \varphi)=\left(\pi_{w_{0}} \psi\right) \varphi=p_{w_{0}} \varphi=\pi_{w_{0}}
$$

and

$$
p_{w_{0}}(\varphi \psi)=\left(p_{w_{0}} \varphi\right) \psi=\pi_{w_{0}} \psi=p_{w_{0}}
$$

whence $\psi \varphi$ and $\varphi \psi$ satisfy respectively the same universal problem as the identity on

$$
\prod_{w \in W} G(w) \text { and } \lim _{w \in W} \Pi^{0}(w),
$$

whence they are mutually inverses. In particular, one has a canonical isomorphism

$$
\lim _{w \in W} \Pi^{0}(w) \cong \prod_{w \in W} G(w)
$$

Now, we want to show that $\Pi^{0}$ is flasque; let $U \subseteq V$ be open subsets of $P$. Using the previous isomorphisms, the natural restriction map

$$
\lim _{V \in V} \Pi^{0}(v) \stackrel{r_{V U}}{\longrightarrow} \lim _{u \in U} \Pi^{0}(u)
$$

can be written as $r_{V U}=\varphi_{U} \circ \pi_{V U} \circ \psi_{V}$, where $\pi_{V U}$ denotes the natural projection

$$
\prod_{v \in V} G(v) \longrightarrow \prod_{u \in U} G(u)
$$


and since $\pi_{V U}$ is surjective (because $U \subseteq V$ ), $r_{V U}$ is also surjective. Summing up, we have checked that $\Pi^{0}$ is a flasque inverse system. Finally, the natural map $G \longrightarrow \Pi^{0}$ clearly defines a monomorphism of $A$-modules; in this way, part (i) holds.

Now, we prove part (ii). Let $I$ be an injective inverse system; by part (i), $I$ can be embedded into a flasque inverse system $\Pi^{0}(I)$. Regardless, since $I$ is injective one has that $I$ is a direct factor of $\Pi^{0}(I)$, whence it is also flasque according to Lemma 2.19. In this way, part (ii) also holds.

We go on proving part (iii). Given an object $G$ of $\operatorname{Inv}(P, \mathcal{A})$, we have constructed in part (i) a flasque inverse system $\Pi^{0}(G)$. Then, setting $Q^{0}:=\operatorname{Coker}\left(G \longrightarrow \Pi^{0}(G)\right)$ we have the following short exact sequence of inverse systems:

$$
0 \longrightarrow G \longrightarrow \Pi^{0}(G) \longrightarrow Q^{0} \longrightarrow 0 \text {. }
$$

Now, replacing $G$ by $Q^{0}$ and setting $\Pi^{1}(G):=\Pi^{0}\left(Q^{0}\right)$ one obtains another short exact sequence:

$$
0 \longrightarrow Q^{0} \longrightarrow \Pi^{1}(G) \longrightarrow Q^{1} \longrightarrow 0
$$

Iterating this process, we build a (possibly infinite) flasque resolution $0 \longrightarrow G \longrightarrow \Pi^{*}$ $(G)$; whence part (iii) also holds.

Finally, part (iv) follows directly combining parts (i), (ii), and (iii) jointly with the fact that flasque inverse systems are acyclic with respect to the limit functor [45, Lemma A.3.9]; the proof is therefore completed.

We conclude this part with the following:

Remark 2.21. Under the equivalence between inverse systems and sheaves of $A$-modules over $P^{o p}$, the resolution of flasque inverse systems (see Definition 2.18) used in the proof of Proposition 2.20 (namely, $\Pi^{*}$ ) corresponds to the so-called Godement resolution [56, Proposition 6.73 and Definition of page 381] for computing sheaf cohomology on the topological space $P^{o p}$; on the other hand, it was already pointed out in $[9,7.2]$ that the equivalence between sheaves and left $A P^{o p}$-modules transforms sheaf cohomology on the topological space $P^{o p}$ into the right derived functors of $\operatorname{Hom}_{A P^{o p}}(A,-)$ (namely, $\left.\operatorname{Ext}_{A P^{o p}}^{*}(A,-)\right)$.

\subsection{Injective, projective, and coflasque direct systems}

It is known that any category of modules over an arbitrary ring has enough injective and projective objects; on the other hand, we have shown in Proposition 2.13 that, whenever 
$P$ is a finite poset, the category $\operatorname{Dir}(P, \mathcal{A})$ is equivalent to the category of left modules over the incidence algebra $I\left(P^{o p}, A\right)$. Therefore, combining these facts one obtains the following:

Theorem 2.22. If $P$ is a finite poset, then the category $\operatorname{Dir}(P, \mathcal{A})$ has enough injective and projective objects.

Remark 2.23. The reader should notice that Theorem 2.22 guarantees the existence of enough projective objects, but it does not provide a priori any information about how these projectives look like.

Now, we face a similar problem to the one considered in the previous subsection; indeed, projective direct systems are not suitable for our homological purposes. For this reason, we have to introduce the following subclass of objects of $\operatorname{Dir}(P, \mathcal{A})$; to the best of our knowledge, the following notion was introduced in [33, Définition 1.I.4].

Definition 2.24. Let $P \stackrel{F}{\longrightarrow} \mathcal{A}$ be a direct system; moreover, we regard $P$ as a topological space with the Alexandrov topology. It is said that $F$ is coflasque if, for any open subset $U \subseteq P$, the natural insertion map $\operatorname{colim}_{u \in U} F(u) \longrightarrow \operatorname{colim}_{p \in P} F(p)$ is injective.

The following result shows that $\operatorname{Dir}(P, \mathcal{A})$ has enough coflasque objects and that this class of direct systems can be used to calculate the left derived functors of the colimit; we refer to [47, pages 53-55, overall Satz 8] for details.

Proposition 2.25. The following statements hold.

(i) Any direct system can be expressed as a homomorphic image of a coflasque direct system.

(ii) Any projective direct system is coflasque.

(iii) Any direct system admits a coflasque resolution.

(iv) The left derived functors of the colimit can be computed through coflasque resolutions.

\section{The Roos Complexes}

Given an inverse system of modules, it was introduced independently by Roos and Nöbeling (see [55] and [47]) a cochain complex that has as $i$ th cohomology the $i$ th right derived functors of the limit. In this subsection, we shall review this definition as well as its dual notion for direct systems. 


\subsection{The homological Roos complex}

We consider a direct system over $P$ valued on $\mathcal{A}$ given by a covariant functor $P \stackrel{F}{\longrightarrow} \mathcal{A}$. Then, we construct a chain complex [30, p. 33]

$$
\cdots \longrightarrow \operatorname{Roos}_{k}(F) \stackrel{d_{k}}{\longrightarrow} \operatorname{Roos}_{k-1}(F) \longrightarrow \cdots
$$

in the following way:

(a) The spots of the complex are

$$
\operatorname{Roos}_{k}(F):=\bigoplus_{p_{0}<\cdots<p_{k}} F_{p_{0} \ldots p_{k}}
$$

where $F_{p_{0} \ldots p_{k}}:=F\left(p_{0}\right) \in \mathcal{A}$.

(b) The boundary map $\operatorname{Roos}_{k}(F) \stackrel{d_{k}}{\longrightarrow} \operatorname{Roos}_{k-1}(F)$ is defined on each direct summand $F_{p_{0} \ldots p_{k}}$ as

$$
j_{p_{1} \ldots p_{k}} \circ F\left(p_{0} \rightarrow p_{1}\right)+\sum_{l=1}^{k}(-1)^{l} j_{p_{0} \ldots \widehat{p}_{l} \ldots p_{k}}
$$

where $j_{p_{0} \ldots p_{k}}$ denotes the natural inclusion map $F_{p_{0} \ldots p_{k}} \longleftrightarrow \operatorname{Roos}_{k}(F)$.

From now on, we denote by $\operatorname{Roos}_{*}(F)$ this chain complex. We collect in the following result the main feature of this construction; we omit its proof because it is completely analogous to the one of Lemma 3.10, which we prove later in this paper.

Lemma 3.1. There is an augmented chain complex $\operatorname{Roos}_{*}(F) \longrightarrow \operatorname{colim}_{p \in P} F(p) \longrightarrow 0$ in the category $\mathcal{A}$; moreover, the homology of this chain complex gives the left derived functors of the colimit, that is, $H_{i}\left(\operatorname{Roos}_{*}(F)\right)=\mathbb{L}_{i} \operatorname{colim}_{p \in P} F(p)$.

Before going on, we would like to write down a small example of $\operatorname{Roos}_{*}(F)$ for the benefit of the reader.

Example 3.2. Let $A$ be a commutative Noetherian ring, let $J \subseteq A$ be an ideal, and let $\Gamma_{J}$ be the corresponding torsion functor. Given an ideal $I \subseteq A$ with primary decomposition $I=I_{1} \cap I_{2}$, consider the poset $P$ given by $I_{1}, I_{2}$, and $I_{1}+I_{2}$, where the order is given by reverse inclusion; in this way, one can define the direct system $P \stackrel{F}{\longrightarrow} \mathcal{A}$ given by $p \longmapsto \Gamma_{I_{p}}(A)$. In this case, the Roos complex $\operatorname{Roos}_{*}(F)$ is as follows. 
On the one hand, the spots of this chain complex are

$$
\operatorname{Roos}_{0}(F)=\Gamma_{I_{1}+I_{2}}(A) \oplus \Gamma_{I_{1}}(A) \oplus \Gamma_{I_{2}}(A) \text { and } \operatorname{Roos}_{1}(F)=\Gamma_{I_{1}+I_{2}}(A) \oplus \Gamma_{I_{1}+I_{2}}(A)
$$

On the other hand, the unique non-zero differential of the complex $d_{1}$ is given by

$$
\begin{aligned}
& \Gamma_{I_{1}+I_{2}}(A) \oplus \Gamma_{I_{1}+I_{2}}(A) \longrightarrow \Gamma_{I_{1}+I_{2}}(A) \oplus \Gamma_{I_{1}}(A) \oplus \Gamma_{I_{2}}(A) \\
& (a, b) \longmapsto(-a-b, a, b) .
\end{aligned}
$$

It is straightforward to check that one has an isomorphism $\operatorname{Coker}\left(d_{1}\right) \cong \operatorname{colim}_{p \in P} \Gamma_{I_{p}}(A)$.

Although the following fact is so elementary, we want to state it because it will play a key role later on (see Setup 4.3).

Lemma 3.3. Assume, in addition, that $F\left(1_{\widehat{P}}\right)$ is defined, that is, that $F$ is not only defined on $P$ but also on $P \cup\left\{1_{\widehat{P}}\right\}$. Then, there is a unique functorial map $\operatorname{colim}_{p \in P} F(p) \stackrel{\psi}{\longrightarrow} F\left(1_{\widehat{P}}\right)$ that makes the following diagram of chain complexes commutative:

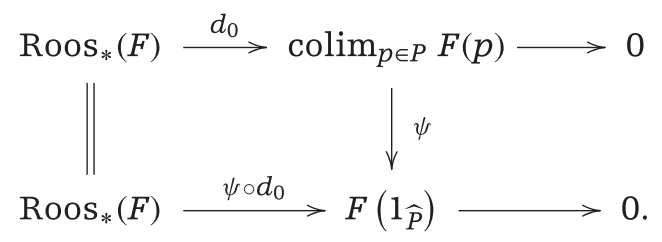

Proof. For any $p \in P, p<1_{\widehat{P}}$ and therefore there is an arrow $F(p) \longrightarrow F\left(1_{\widehat{P}}\right)$. In this way, the universal property of the colimit produces $\psi$ with the desired properties.

Remark 3.4. Preserving the assumptions of Lemma 3.3, in general $\psi$ does not define an isomorphism. Equivalently, in general it is not true that the natural map

$$
\operatorname{colim}_{p \in P} F(p) \stackrel{\psi}{\longrightarrow} \operatorname{colim}_{p \in \widehat{P}} F(p)=F\left(1_{\widehat{P}}\right)
$$

is bijective. It is noteworthy that the equality $\operatorname{colim}_{p \in \widehat{P}} F(p)=F\left(1_{\widehat{P}}\right)$ is well known [56, Exercise 5.22 (iii)].

Before going on, we need to introduce the following: 
Definition 3.5. We define the rank of $P$ (namely, $\operatorname{rank}(P))$ in the following way:

$$
\operatorname{rank}(P):=\left(\max _{p \in P} \#\left[p, 1_{\widehat{P}}\right)\right)-1
$$

Remark 3.6. Let $\Delta(P)$ be the order complex associated to $P$; by the very definition of the order complex of a poset, it follows that $\operatorname{rank}(P)=\operatorname{dim}(\Delta(P))$, where by $\operatorname{dim}(\Delta(P))$ we mean its dimension as simplicial complex.

Keeping in mind the notion of rank of a poset, we are ready to establish another important property of the Roos chain complex that we plan to exploit later on (see proof of Theorem 4.6); namely the following:

Lemma 3.7. If $k>\operatorname{rank}(P)$, then $\operatorname{Roos}_{k}(F)=0$ for any direct system $F$; in particular, one has $\mathbb{L}_{k} \operatorname{colim}_{p \in P} F(p)=0$.

Proof. Fix $k>\operatorname{rank}(P)$ and any direct system $F$. Since $k>\operatorname{rank}(P)$ there are no ordered chains $p_{0}<\cdots<p_{k}$ in $P$, and therefore

$$
\operatorname{Roos}_{k}(F)=\bigoplus_{p_{0}<\cdots<p_{k}} F_{p_{0} \ldots p_{k}}=0
$$

as required. Then $\mathbb{L}_{k} \operatorname{colim}_{p \in P} F(p)=0$ follows directly from Lemma 3.1.

Another quite important (but well-known) property of the Roos chain complex that we use several times in this paper is the following:

Lemma 3.8. Let $G$ be an abelian group, and let $|G|$ be the constant direct system on $\widehat{P}$ given by $G$ with identities on $G$ as structural morphisms. Then, $H_{i}\left(\operatorname{Roos}_{*}(|G|)\right)=\widetilde{H}_{i}(P ; G)$. In other words, $\operatorname{Roos}_{*}(|G|)$ may be regarded as a chain complex for computing the reduced simplicial homology of the topological space $P$ (regarding $P$ as a topological space with the Alexandrov topology) with coefficients in $G$.

Proof. Notice that $\widetilde{H}_{i}(P ; G)=\widetilde{H}_{i}(\Delta(P) ; G)$, because the order complex $\Delta(P)$ provides a triangulation of $P$ as topological space; moreover, $\widetilde{H}_{i}(\Delta(P) ; G)=H_{i}\left(\widetilde{\mathcal{C}}_{\bullet}(\Delta(P)) \otimes_{\mathbb{Z}} G\right)$, where $\widetilde{\mathcal{C}}_{\bullet}(\Delta(P))$ denotes the chain complex for computing the reduced simplicial homology of $\Delta(P)$ with integer coefficients. In this way, the result follows from the fact that $\widetilde{\mathcal{C}}_{\bullet}(\Delta(P)) \otimes_{\mathbb{Z}} G=\operatorname{Roos}_{*}(|G|)$. 
We end this subsection with the following remark, which can be proved by just using the very definition of the Roos chain complex (essentially, that its spots consist of finite direct sums):

Remark 3.9. We denote by $\operatorname{Ch}(\mathcal{A})$ the category of chain complexes of objects of $\mathcal{A}$. In this way, we just constructed a functor $\operatorname{Dir}(P, \mathcal{A}) \stackrel{\operatorname{Roos}_{*}}{\longrightarrow} \mathrm{Ch}(\mathcal{A})$ that is exact and commutes with direct sums.

\subsection{The cohomological Roos complex}

Now, we consider an inverse system over $P$ valued on $\mathcal{A}$ given by a contravariant functor $P \stackrel{G}{\longrightarrow} \mathcal{A}$. Thus, we build a cochain complex of inverse systems $\cdots \longrightarrow \operatorname{Roos}^{k}(G) \stackrel{d^{k}}{\longrightarrow} \operatorname{Roos}^{k+1}(G) \longrightarrow \cdots$ as follows (see either [30, pp. 31-32] or [45, pp. 346-347]).

(a) The pieces of the complex are

$$
\operatorname{Roos}^{k}(G):=\prod_{p_{0}<\cdots<p_{k}} G_{p_{0} \ldots p_{k}}
$$

where $G_{p_{0} \ldots p_{k}}:=G\left(p_{0}\right)$.

(b) The coboundary map $\operatorname{Roos}^{k}(G) \stackrel{d^{k}}{\longrightarrow} \operatorname{Roos}^{k+1}(G)$ is defined on each factor $G_{p_{0} \ldots p_{k}}$ as

$$
G\left(p_{0} \rightarrow p_{1}\right) \circ \pi_{p_{0} \ldots p_{k}}+\sum_{l=1}^{k+1}(-1)^{l} \pi_{p_{0} \ldots \widehat{p}_{l} \ldots p_{k+1}},
$$

where $\pi_{p_{0} \ldots p_{k}}$ denotes the natural projection $\operatorname{Roos}^{k}(G) \longrightarrow G_{p_{0} \ldots p_{k}}$.

Hereafter, we shall denote by $\operatorname{Roos}^{*}(G)$ this cochain complex. As in the homological case, the main feature of this cohomological construction is reviewed in the following result; in this case, we provide a proof for the reader's convenience (cf. [45, Proof of Lemma A.3.2]).

Lemma 3.10. There is a coaugmented cochain complex $0 \longrightarrow \lim _{p \in P} G(p) \longrightarrow \operatorname{Roos}^{*}(G)$ in the category $\mathcal{A}$; moreover, the cohomology of this cochain complex yields the right derived functors of the limit, that is, $H^{i}\left(\operatorname{Roos}^{*}(G)\right)=\mathbb{R}^{i} \lim _{p \in P} G(p)$.

Proof. What we show is that the right derived functors of the limit $\left(U^{j}\right)_{j \geq 0}:=$ $\left(\mathbb{R}^{j} \lim _{p \in P}\right)_{j \geq 0}$ and the cohomology of the Roos cochain complex $\left(V^{j}\right)_{j \geq 0}:=\left(H^{j}\left(\operatorname{Roos}^{*}(-)\right)\right)_{j \geq 0}$ are isomorphic universal $\delta$-functors. 
Indeed, on the one hand it is clear that both $\left(U^{j}\right)_{j \geq 0}$ and $\left(V^{j}\right)_{j \geq 0}$ are universal $\delta$-functors with

$$
U^{0}=\mathbb{R}^{0} \lim _{p \in P}=\lim _{p \in P}=H^{0}\left(\operatorname{Roos}^{*}(-)\right)=V^{0} .
$$

On the other hand, given any $j \geq 1$ and any injective inverse system $I$ it is also clear that

$$
U^{j}(I)=\mathbb{R}^{j} \lim _{p \in P} I_{p}=0=H^{j}\left(\operatorname{Roos}^{*}(I)\right)=V^{j}(I)
$$

notice that the equality $0=H^{j}\left(\operatorname{Roos}^{*}(I)\right)$ is a direct application of [47, Satz 6]. Combining the previous two facts one obtains a canonical isomorphism

$$
\left(\mathbb{R}^{j} \lim _{p \in P}\right)_{j \geq 0} \cong\left(H^{j}\left(\operatorname{Roos}^{*}(-)\right)\right)_{j \geq 0}
$$

of universal $\delta$-functors, just what we finally wanted to check.

Before going on, we would like to write down a small example of $\operatorname{Roos}^{*}(G)$ for the benefit of the reader.

Example 3.11. Let $A$ be a commutative Noetherian ring; given an ideal $I \subseteq A$ with primary decomposition $I=I_{1} \cap I_{2}$, consider the poset $P$ given by $I_{1}, I_{2}$, and $I_{1}+I_{2}$, where the order is given by reverse inclusion. In this way, one can define the inverse system $P \stackrel{G}{\longrightarrow} \mathcal{A}$ given by $p \longmapsto A / I_{p}$. In this case, the Roos complex $\operatorname{Roos}^{*}(G)$ is as follows:

On the one hand, the spots of this cochain complex are

$$
\operatorname{Roos}^{0}(G)=A /\left(I_{1}+I_{2}\right) \times A / I_{1} \times A / I_{2} \text { and } \operatorname{Roos}^{1}(G)=A /\left(I_{1}+I_{2}\right) \times A /\left(I_{1}+I_{2}\right) .
$$

On the other hand, the unique non-zero differential of the complex $d^{0}$ is given by

$$
\begin{aligned}
& A /\left(I_{1}+I_{2}\right) \times A / I_{1} \times A / I_{2} \longrightarrow A /\left(I_{1}+I_{2}\right) \times A /\left(I_{1}+I_{2}\right) \\
& (a, b, c) \longmapsto(-a+b,-a+c) .
\end{aligned}
$$


In this case, it is straightforward to check that the constant map

$$
\begin{aligned}
& A / I \longrightarrow A /\left(I_{1}+I_{2}\right) \times A / I_{1} \times A / I_{2} \\
& a \longmapsto(a, a, a) .
\end{aligned}
$$

establishes an isomorphism $\lim _{p \in P} A / I_{p}=\operatorname{ker}\left(d^{0}\right) \cong A / I$.

We state the cohomological analogous of Lemma 3.3. We skip the details.

Lemma 3.12. Suppose, in addition, that $G\left(1_{\widehat{P}}\right)$ is defined; that is, that $G$ is not only defined on $P$ but also in $P \cup\left\{1_{\widehat{P}}\right\}$. Then, there is a unique functorial map $G\left(1_{\widehat{P}}\right) \stackrel{\alpha}{\longrightarrow} \lim _{p \in P} G(p)$ that makes commutative the below diagram of cochain complexes

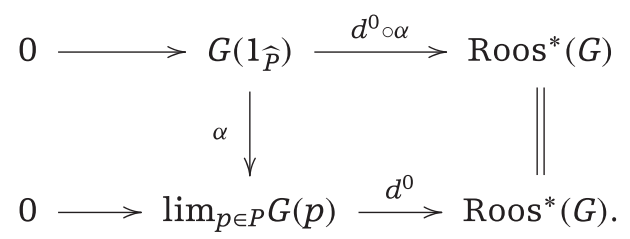

Remark 3.13. Preserving the assumptions of Lemma 3.12, it is in general not true that $\alpha$ defines an isomorphism. In other words, it is in general not true that the natural restriction map

$$
G\left(1_{\widehat{P}}\right)=\lim _{p \in \widehat{P}} G(p) \stackrel{\alpha}{\longrightarrow} \lim _{p \in P} G(p)
$$

is an isomorphism. It is known (see [9, 3.3] and [10, Theorem 5.6]) to be an isomorphism in some particular situations.

Next, we state the cohomological analogous of Lemma 3.7; once again, the details are left to the interested reader.

Lemma 3.14. If $k>\operatorname{rank}(P)$, then $\operatorname{Roos}^{k}(G)=0$ for any inverse system $G$; in particular, $\mathbb{R}^{k} \lim _{p \in P} G(p)=0$.

Now, we write down the cohomological analogous of Lemma 3.8; once again, we omit the details.

Lemma 3.15. Let $G$ be an abelian group, and let $|G|$ be the constant inverse system on $\widehat{P}$ given by $G$ with identities on $G$ as structural morphisms. Then, $H^{i}\left(\operatorname{Roos}^{*}(|G|)\right)=\widetilde{H}^{i}(P ; G)$. 
In other words, $\operatorname{Roos}^{*}(|G|)$ may be regarded as a cochain complex for computing the reduced simplicial cohomology of the topological space $P$ (regarding $P$ as a topological space with the dual Alexandrov topology) with coefficients in $G$.

As in the homological case, we end with the following:

Remark 3.16. If we denote by $\operatorname{CoCh}(\mathcal{A})$ the category of cochain complexes of objects of $\mathcal{A}$, then we have produced a functor $\operatorname{Inv}(P, \mathcal{A}) \stackrel{\operatorname{Roos}^{*}}{\rightarrow} \operatorname{CoCh}(\mathcal{A})$ that is exact and commutes with direct products.

\section{Homological Spectral Sequences Associated to Modules}

Hereafter, $A$ will denote a commutative Noetherian ring and $\mathcal{A}$ will stand for the category of $A$-modules. To any ideal $I \subseteq A$ one may associate the poset $P$ given in Example 2.1. Namely, let $I=I_{1} \cap \ldots \cap I_{n}$ be a decomposition of $I$ into $n$ ideals, then $P$ is the poset given by all the possible sums of the ideals $I_{1}, \ldots, I_{n}$ ordered by reverse inclusion; but we underline that we have to identify these sums when they describe the same ideal.

The aim of this section is to construct several spectral sequences even though we will be mostly interested on those involving local cohomology modules; as we have already pointed out in the introduction, the spectral sequences we construct in this section involve in their 2nd page the left derived functors of the colimit, for this reason we refer to them as homological. The method will be as follows: given any object $M$ of $\mathcal{A}$, we shall construct a direct system over $P$ of objects of $\mathcal{A}$. Then, using the homological Roos complex we shall build a double complex with a finite number of nonzero columns (or rows) that will rise to a spectral sequence that, with the help of a technical lemma, will converge to a certain object of $\mathcal{A}$.

Before moving to our promised construction, we want to review now the following:

Definition 4.1. Given $J, K \subseteq A$ ideals, set $\mathrm{W}(K, J):=\left\{\mathfrak{q} \in \operatorname{Spec}(A) \mid K^{n} \subseteq \mathfrak{q}+J\right.$ for some integer $n \geq 1$ \}. Remember (see [61]) that, when $J=(0), \mathbf{W}(K, J)=\mathbf{V}(K)$.

We also want to single out in the next result some elementary properties that the subsets of the form $\mathbf{W}(K, J)$ verify because they will play a crucial role later on (see Lemma 4.4); the below lemma is just a piece of $[61,1.6]$, and since its proof is straightforward, we omit it. 
Lemma 4.2. Let $I$ and $I^{\prime}$ be ideals of $A$, let $\mathfrak{p} \in \operatorname{Spec}(A)$, and let $J$ be an ideal of $A$. Then, the following statements hold.

(i) If $\mathfrak{p} \in \mathrm{W}(I, J) \cap \mathrm{W}\left(I^{\prime}, J\right)$, then $\mathfrak{p} \in \mathrm{W}\left(I+I^{\prime}, J\right)$.

(ii) If $I^{\prime} \subseteq I$ and $\mathfrak{p} \in \mathbf{W}(I, J)$, then $\mathfrak{p} \in \mathbf{W}\left(I^{\prime}, J\right)$.

\subsection{Construction of homological spectral sequences}

The setup we will need to build the announced spectral sequences is the following direct system of functors that we plan to describe with detail in the next:

Setup 4.3. Let $\mathcal{A} \stackrel{T_{[*]}}{\longrightarrow} \operatorname{Dir}(\widehat{P}, \mathcal{A})$ be an additive, covariant functor that verifies the following requirements:

(i) For any $p \in \widehat{P}, T_{p}$ is a covariant, left exact functor that commutes with direct sums.

(ii) If $p \leq q$ then there exists a natural transformation of functors $T_{p} \stackrel{\psi_{p q}}{\longrightarrow} T_{q}$; notice that this natural transformation extends in a unique way to a natural transformation $\mathbb{R}^{i} T_{p} \stackrel{\psi_{p q}^{i}}{\longrightarrow} \mathbb{R}^{i} T_{q}$ for any $i \geq 0$ such that $\psi_{p q}^{0}=\psi_{p q}$ (see $[8,1.3 .4$ and 1.3.5] for details).

(iii) There exists an ideal $J$ of $A$ such that the following holds: for any $\mathfrak{p} \in \operatorname{Spec}(A)$ and for any maximal ideal $\mathfrak{m}$ of $A$, there exist $A$-modules $X$ and $Y$ such that, for any $p \in \widehat{P}$,

$$
T_{p}(E(A / \mathfrak{p}))_{\mathfrak{m}}=\left\{\begin{array}{l}
X, \text { if } \mathfrak{p} \in \mathbf{W}\left(I_{p}, J\right) \text { and } \mathfrak{p} \subseteq \mathfrak{m}, \\
Y, \text { if } \mathfrak{p} \notin \mathbf{W}\left(I_{p}, J\right) \text { and } \mathfrak{p} \subseteq \mathfrak{m} \\
0, \text { otherwise. }
\end{array}\right.
$$

Here, $X$ and $Y$ may depend on $J, \mathfrak{p}$, and $\mathfrak{m}$ but not on $p$. Moreover, given $p \leq q$,

$$
T_{p}(E(A / \mathfrak{p}))_{\mathfrak{m}} \longrightarrow T_{q}(E(A / \mathfrak{p}))_{\mathfrak{m}}=\left\{\begin{array}{l}
\mathbb{1}_{X}, \text { if } T_{p}(E(A / \mathfrak{p}))_{\mathfrak{m}}=X=T_{q}(E(A / \mathfrak{p}))_{\mathfrak{m}} \\
\mathbb{1}_{Y,} \text { if } T_{p}(E(A / \mathfrak{p}))_{\mathfrak{m}}=Y=T_{q}(E(A / \mathfrak{p}))_{\mathfrak{m}} \\
0, \text { otherwise. }
\end{array}\right.
$$

Hereafter in this subsection, under the assumptions of Setup 4.3 we set $T:=T_{1_{\hat{P}}}$; moreover, we also define the cohomological dimension of $T$, denoted by $\operatorname{cd}(T)$, in the 
following way:

$$
\operatorname{cd}(T):=\max \left\{i \in \mathbb{N} \mid \mathbb{R}^{i} T(X) \neq 0 \text { for some } A-\text { module } X\right\}
$$

Moreover, given an $A$-module $M, \mathbb{R}^{i} T_{[*]}(M)$ will denote the direct system $\left(\mathbb{R}^{i} T_{p}(M)\right)_{p \in P}$ with structural maps $\mathbb{R}^{i} T_{p}(M) \longrightarrow \mathbb{R}^{i} T_{q}(M)$ for $p \leq q$.

\subsubsection{Main result}

In order to compute the abutment of our homological spectral sequences we will need the following lemma, which may be regarded as a generalization of [4, Lemma of p. 38].

Lemma 4.4. If $E$ is any injective $A$-module, then the augmented homological Roos chain complex $\operatorname{Roos}_{*}\left(T_{[*]}(E)\right) \longrightarrow T(E) \longrightarrow 0$ is exact.

Proof. First of all, as $\operatorname{Roos}_{*}$ and $T_{[*]}$ commute with direct sums we deduce from the Matlis-Gabriel theorem [8, 10.1.9] that we may assume, without loss of generality, that there is $\mathfrak{p} \in \operatorname{Spec}(A)$ such that $E=E_{A}(A / \mathfrak{p})$, a choice of injective hull of $A / \mathfrak{p}$ over $A$. Moreover, as being exact is a local property it is enough to check that, for any maximal ideal $\mathfrak{m}$ of $A$, the chain complex

$$
\operatorname{Roos}_{*}\left(T_{[*]}(E)\right)_{\mathfrak{m}} \longrightarrow T(E)_{\mathfrak{m}} \longrightarrow 0
$$

is exact. Indeed, we can express $P$ as the disjoint union of $Q$ and $Q^{\prime}$, where

$$
\begin{aligned}
& Q:=\left\{q \in P \mid \quad I_{q} \subseteq \mathfrak{m}\right\}, \\
& Q^{\prime}:=\left\{q \in P \mid \quad I_{q} \nsubseteq \mathfrak{m}\right\} .
\end{aligned}
$$

Notice that $Q$ is clearly a subposet of $P$ (this just means that $Q$ is a subset of $P$ that is also a poset with the same partial order of $P$ ).

Now, assume that $Y=0$; we have to distinguish two cases. Firstly, if $\mathfrak{p} \nsubseteq \mathfrak{m}$, then the previous chain complex is identically zero, whence we are done. Otherwise, suppose that $\mathfrak{p} \subseteq \mathfrak{m}$; in this case, we split $Q$ as the disjoint union $Q=Q_{1} \cup Q_{2}$, where

$$
\begin{aligned}
& Q_{1}:=\left\{p \in Q \mid \mathfrak{p} \in W\left(I_{p}, J\right)\right\} \\
& Q_{2}:=\left\{p \in Q \mid \mathfrak{p} \notin W\left(I_{p}, J\right)\right\} .
\end{aligned}
$$


Now, we have to distinguish the two cases. Indeed, if $\mathfrak{p} \notin W\left(I_{p}, J\right)$ then the previous chain complex is identically zero and therefore we are done. Otherwise, suppose that $\mathfrak{p} \in W\left(I_{p}, J\right)$ for at least one $p$; in this case, this assumption combined with Lemma 4.2 ensures that $Q_{1}$ is a nonempty subposet of $P$ of the form $\left[r, 1_{\widehat{P}}\right)$, where $r \in P$ such that

$$
I_{r}=\sum_{q \in O_{1}} I_{q}:=J
$$

Indeed, since the ideal $J$ is clearly the greatest ideal among the ideals of $Q_{1}$ (we want to stress that here is where we are using the finiteness of $O_{1}$ combined with Lemma 4.2), it turns out that there is an element $r \in P$ such that $I_{r}=J$ and therefore $Q_{1}=\left[r, 1_{\widehat{P}}\right)$. Summing up, our chain complex

$$
\operatorname{Roos}_{*}\left(T_{[*]}(E)\right)_{\mathfrak{m}} \longrightarrow T(E)_{\mathfrak{m}} \longrightarrow 0
$$

agrees with the one obtained considering the Roos chain complex on $Q_{1}$ instead of $P$; finally, this augmented chain complex where we only consider the subposet $Q_{1}$ in the construction of the Roos chain complex equals the augmented one for computing the simplicial homology of the topological space $\left[r, 1_{\widehat{P}}\right.$ ) with coefficients in $X$ (see Lemma 3.8). But we have checked in Lemma 2.9 that this topological space is contractible. This concludes the proof provided that $Y=0$.

Now, assume that $X=0$ and that $\mathfrak{p} \subseteq \mathfrak{m}$; now, consider the short exact sequence of direct systems

$$
0 \longrightarrow|Y|_{\mid P_{1}} \longrightarrow|Y| \longrightarrow|Y|_{\mid P-P_{1}} \longrightarrow 0 \text {. }
$$

Here, $P_{1}:=\left\{p \in P \mid \mathfrak{p} \in W\left(I_{p}, J\right), I_{p} \subseteq \mathfrak{m}\right\}$ and $|Y|_{\mid P_{1}}$ (respectively, $\left.|Y|_{\mid P-P_{1}}\right)$ denotes the direct system with constant value $Y$ in all the points of $P_{1}$ (respectively, $P-P_{1}$ ) and zero elsewhere; moreover, its nonzero structural homomorphisms are all identities on $Y$. As we have seen in the 1 st part of this proof, we can write $P_{1}=\left[r, 1_{\widehat{P}}\right)$ for some $r \in P$. On the other hand, (3) induces the following short exact sequence of chain complexes:

$$
0 \longrightarrow \operatorname{Roos}_{*}\left(|Y|_{\mid P_{1}}\right) \longrightarrow \operatorname{Roos}_{*}(|Y|) \longrightarrow \operatorname{Roos}_{*}\left(|Y|_{\mid P-P_{1}}\right) \longrightarrow 0
$$

Now, since $X=0$ it follows that $\operatorname{Roos}_{*}\left(|Y|_{\mid P-P_{1}}\right)=\operatorname{Roos}_{*}\left(T_{[*]}(E)\right)_{\mathfrak{m}}$, whence we can rewrite (4) in the following manner:

$$
0 \longrightarrow \operatorname{Roos}_{*}\left(|Y|_{\mid P_{1}}\right) \longrightarrow \operatorname{Roos}_{*}(|Y|) \longrightarrow \operatorname{Roos}_{*}\left(T_{[*]}(E)\right)_{\mathfrak{m}} \longrightarrow 0
$$


However, $\operatorname{Roos}_{*}\left(|Y|_{\mid P_{1}}\right)$ (respectively, $\left.\operatorname{Roos}_{*}(|Y|)\right)$ turns out to be the chain complex for computing the reduced simplicial homology of the topological space $P_{1}=\left[r, 1_{\widehat{P}}\right)$ (respectively, $P$ ) (see Lemma 3.8); since both $P_{1}$ and $P$ are contractible (see Lemma 2.9) one has that both $\operatorname{Roos}_{*}\left(|Y|_{\mid P_{1}}\right)$ and $\operatorname{Roos}_{*}(|Y|)$ are exact.

Summing up, all the foregoing implies that (5) is a short exact sequence of chain complexes with two of them being exact; whence the remaining one (namely, $\left.\operatorname{Roos}_{*}\left(T_{[*]}(E)\right)_{\mathfrak{m}}\right)$ is also exact, just what we wanted to prove.

Finally, assume that both $X$ and $Y$ are nonzero and that $\mathfrak{p} \subseteq \mathfrak{m}$; as before, $P_{1}:=$ $\left\{p \in P \mid \mathfrak{p} \in W\left(I_{p}, J\right), I_{p} \subseteq \mathfrak{m}\right\}$ can be written as $P_{1}=\left[r, 1_{\widehat{P}}\right)$ for some $r \in P$. Thus, we have the following short exact sequence of direct systems:

$$
0 \longrightarrow|X|_{\mid P_{1}} \longrightarrow T_{[*]}(E)_{\mathfrak{m}} \longrightarrow|Y|_{\mid P-P_{1}} \longrightarrow 0
$$

Since $\operatorname{Roos}_{*}$ is exact, this short exact sequence of direct systems induces the following short exact sequence of chain complexes:

$$
0 \longrightarrow \operatorname{Roos}_{*}\left(|X|_{\mid P_{1}}\right) \longrightarrow \operatorname{Roos}_{*}\left(T_{[*]}(E)_{\mathfrak{m}}\right) \longrightarrow \operatorname{Roos}_{*}\left(|Y|_{\mid P-P_{1}}\right) \longrightarrow 0
$$

But we have seen during this proof that both $\operatorname{Roos}_{*}\left(|X|_{\mid P_{1}}\right)$ and $\operatorname{Roos}_{*}\left(|Y|_{\mid P-P_{1}}\right)$ are exact, hence $\operatorname{Roos}_{*}\left(|Y|_{\mid P-P_{1}}\right)$ is also exact. The proof is therefore completed.

Remark 4.5. Notice that, given any $A$-module $M$, it is not true, in general, that $T(M)$ is isomorphic to colim ${ }_{p \in P} T_{p}(M)$; however, Lemma 4.4 shows, in particular, that if $E$ is any injective $A$-module, then $T(E) \cong \operatorname{colim}_{p \in P} T_{p}(E)$.

Our next statement is the main result of this section.

Theorem 4.6. Let $M$ be any $A$-module. Then, we have the following spectral sequence

$$
E_{2}^{-i, j}=\mathbb{L}_{i} \operatorname{colim}_{p \in P} \mathbb{R}^{j} T_{[*]}(M) \Longrightarrow \mathbb{R}^{j-i} T(M)
$$

in the category of $A$-modules.

Proof. Let $0 \longrightarrow M \longrightarrow E^{*}$ be an injective resolution of $M$ in the category $\mathcal{A}$. Applying to this resolution the functor $T_{[*]}$ one gets the following cochain complex of direct 
systems:

$$
0 \longrightarrow T_{[*]}(M) \longrightarrow T_{[*]}\left(E^{0}\right) \longrightarrow T_{[*]}\left(E^{1}\right) \longrightarrow \ldots
$$

Now, we use the Roos chain complex in order to produce the bicomplex $\operatorname{Roos}^{-i}\left(T_{[*]}\left(E^{j}\right)\right)=$ $\operatorname{Roos}_{i}\left(T_{[*]}\left(E^{j}\right)\right)$; the reader will easily note that we put minus in the index $i$ because we want to work with a bicomplex located in the 2nd quadrant. We hope the following picture illustrates this sentence:

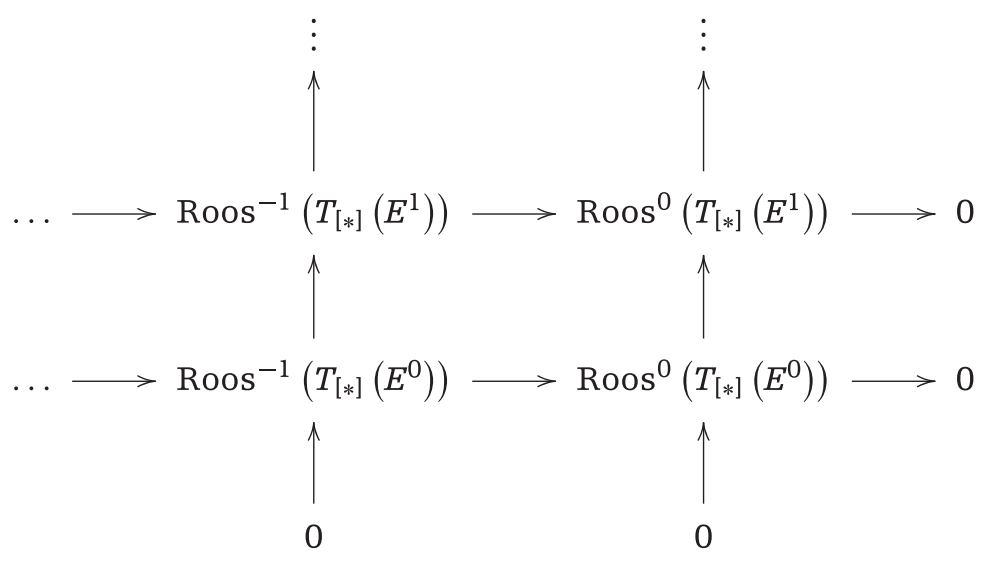

Moreover, we have to stress that the horizontal differentials are the ones of the Roos chain complex and the vertical ones are the induced by the injective resolution of $M$; so, the bicomplex $\operatorname{Roos}^{-i}\left(T_{[*]}\left(E^{j}\right)\right)$ produces two spectral sequences. Namely, the ones provided by the 1 st and the 2nd filtration of the previous bicomplex, respectively. In this way, the 1st thing one should ensure is that both spectral sequences converge and calculate their common abutment.

First of all, we want to check that our spectral sequences converge; indeed, Lemma 3.7 ensures that $\operatorname{Roos}^{-i}\left(T_{[*]}\left(E^{j}\right)\right)=0$ for all $i \gg 0$. Therefore, this implies that the bicomplex $\operatorname{Roos}^{-i}\left(T_{[*]}\left(E^{j}\right)\right)$ has just a finite number of columns, which implies the convergence of both spectral sequences.

Second, notice that the $E_{2}$-page of one of these spectral sequences is obtained by firstly computing the cohomology of the rows and then computing the cohomology of the columns; regardless, Lemma 4.4 guarantees that all the rows of the bicomplex $\operatorname{Roos}^{-i}\left(T_{[*]}\left(E^{j}\right)\right)$ are exact up to the 0th spot. Therefore, this fact implies that this spectral sequence collapses, whence its abutment turns out to be $\mathbb{R}^{*} T(M)$.

Finally, the other spectral sequence that we can produce is the one obtained by firstly taking cohomology on the columns and then calculating the cohomology of the rows; in this case, one obtains as $E_{1}$-page $E_{1}^{-i, j}=\operatorname{Roos}^{-i}\left(\mathbb{R}^{j} T_{[*]}(M)\right)$. In addition, since the boundary map of the $E_{1}$-page is the one of the Roos chain complex, and this chain 
complex computes the $i$ th left derived functor of the colimit, its $E_{2}$-page turns out to be $E_{2}^{-i, j}=\mathbb{L}_{i} \operatorname{colim}_{p \in P} \mathbb{R}^{j} T_{[*]}(M)$. Summing up, combining all the foregoing facts one obtains the spectral sequence

$$
E_{2}^{-i, j}=\mathbb{L}_{i} \operatorname{colim}_{p \in P} \mathbb{R}^{j} T_{[*]}(M) \Longrightarrow \mathbb{R}^{j-i} T(M)
$$

in the category of $A$-modules; the proof is therefore completed.

\subsection{Examples}

We provide several examples of local cohomology spectral sequences. In particular, the corresponding functors verify the established conditions in Setup 4.3. In all of these examples, $N$ will denote a finitely generated $A$-module.

- Generalized local cohomology: The direct system of functors $T_{[*]}=\Gamma_{[*]}(N,-)$ of generalized torsion functors given by $T_{p}(M):=\Gamma_{I_{p}}(N, M)$ verifies all requirements established in Setup 4.3; moreover, in this case $T=\Gamma_{I}(N,-)$. Indeed, first of all we remember that, for any $p \in \widehat{P}$ and for any $A$-module $M$,

$$
H_{I_{p}}^{j}(N, M):=\operatorname{colim}_{k \in \mathbb{N}} \operatorname{Ext}_{A}^{j}\left(N / I_{p}^{k} N, M\right)
$$

Now, fix $k \in \mathbb{N}$. Using the adjoint associativity between Hom and tensor product [56, 2.76] one has that $\operatorname{Hom}_{A}\left(N / I_{p}^{k} N, E(A / \mathfrak{p})\right) \cong \operatorname{Hom}_{A}\left(N, \operatorname{Hom}_{A}\left(A / I_{p}^{k}, E(A / \mathfrak{p})\right)\right)$. On the other hand, since $N$ is finitely generated it follows that

$$
\operatorname{colim}_{k \in \mathbb{N}} \operatorname{Hom}_{A}\left(N, \operatorname{Hom}_{A}\left(A / I_{p}^{k}, E(A / \mathfrak{p})\right)\right) \cong \operatorname{Hom}_{A}\left(N, \operatorname{colim}_{k \in \mathbb{N}} \operatorname{Hom}_{A}\left(A / I_{p}^{k}, E(A / \mathfrak{p})\right)\right)
$$

Summing up, one has that $\operatorname{colim}_{k \in \mathbb{N}} \operatorname{Hom}_{A}\left(N / I_{p}^{k} N, E(A / \mathfrak{p})\right) \cong \operatorname{Hom}_{A}\left(N, \Gamma_{I_{p}}(E(A / \mathfrak{p}))\right)$. Moreover, it is well known $[8,10.1 .11]$ that

$$
\Gamma_{I_{p}}(E(A / \mathfrak{p}))=\left\{\begin{array}{l}
E(A / \mathfrak{p}), \text { if } \mathfrak{p} \in \mathrm{V}\left(I_{p}\right) \\
0, \text { if } \mathfrak{p} \notin \mathrm{V}\left(I_{p}\right)
\end{array}\right.
$$

In this way, combining these two facts it follows that

$$
\Gamma_{I_{p}}(N, E(A / \mathfrak{p})) \cong \operatorname{Hom}_{A}\left(N, \Gamma_{I_{p}}(E(A / \mathfrak{p}))\right)=\left\{\begin{array}{l}
\operatorname{Hom}_{A}(N, E(A / \mathfrak{p})), \text { if } \mathfrak{p} \in \mathrm{V}\left(I_{p}\right) \\
0, \text { if } \mathfrak{p} \notin \mathrm{V}\left(I_{p}\right)
\end{array}\right.
$$


Finally, given any $\mathfrak{m} \in \operatorname{Max}(A)$ one has, as a direct application of [8, 4.1.7] (indeed, $N$ is finitely generated and the localization map $A \longrightarrow A_{\mathfrak{m}}$ is flat), that

$$
\Gamma_{I_{p}}(N, E(A / \mathfrak{p}))_{\mathfrak{m}}=\left\{\begin{array}{l}
\operatorname{Hom}_{A_{\mathfrak{m}}}\left(N_{\mathfrak{m}}, E(A / \mathfrak{p})_{\mathfrak{m}}\right), \text { if } \mathfrak{p} \in \mathrm{V}\left(I_{p}\right) \text { and } \mathfrak{p} \subseteq \mathfrak{m} \\
0, \text { otherwise. }
\end{array}\right.
$$

Therefore, we get the following spectral sequence:

$$
E_{2}^{-i, j}=\mathbb{L}_{i} \operatorname{colim}_{p \in P} H_{I_{p}}^{j}(N, M) \Longrightarrow H_{I}^{j-i}(N, M)
$$

This spectral sequence is a generalization of [4, p. 39] and [37, 2.1]. In case the ideal has two components $I=I_{1} \cap I_{2}$, we recover partially the Mayer-Vietoris long exact sequence obtained in [67, 2.14]:

$$
\ldots \longrightarrow H_{I_{1}}^{j}(N, M) \oplus H_{I_{2}}^{j}(N, M) \longrightarrow H_{I}^{j}(N, M) \longrightarrow H_{I_{1}+I_{2}}^{j+1}(N, M) \longrightarrow \ldots
$$

For the sake of simplicity, we single out the version of it that we shall consider in this paper, especially for the case when $N=A$ is the ring itself.

$$
E_{2}^{-i, j}=\mathbb{L}_{i} \operatorname{colim}_{p \in P} H_{I_{p}}^{j}(M) \Longrightarrow H_{I}^{j-i}(M)
$$

It is also worth mentioning that the Mayer-Vietoris spectral sequence considered in $[37,2.1]$ is slightly different at the $E_{1}$-page (because he used a different poset associated to the ideal $I$ ) but they coincide at the $E_{2}$-page; indeed, it follows from the fact that our poset is cofinal with respect to the one considered in $[37,2.1]$.

- Generalized ideal transform: The direct system of functors $T_{[*]}=D_{[*]}(N,-)$ of generalized Nagata's ideal transforms verifies all the requirements [16]. In this case, $T=D_{I}(N,-)$ and recall that by definition, given $p \in \widehat{P}$, we have $D_{I_{p}}(N, M):=$ $\operatorname{colim}_{k \in \mathbb{N}} \operatorname{Hom}_{A}\left(I_{p}^{k} N, M\right)$ for any $A$-module $M$. By $[16,2.2]$, there is an exact sequence

$$
0 \longrightarrow \Gamma_{I_{p}}(N, E(A / \mathfrak{p})) \longrightarrow \operatorname{Hom}_{A}(N, E(A / \mathfrak{p})) \longrightarrow D_{I_{p}}(N, E(A / \mathfrak{p})) \longrightarrow H_{I_{p}}^{1}(N, E(A / \mathfrak{p}))
$$

So, since $E(A / \mathfrak{p})$ is injective, it follows that $H_{I_{p}}^{1}(N, E(A / \mathfrak{p}))=0$, whence one can arrange the previous exact sequence in the following way:

$$
0 \longrightarrow \Gamma_{I_{p}}(N, E(A / \mathfrak{p})) \longrightarrow \operatorname{Hom}_{A}(N, E(A / \mathfrak{p})) \longrightarrow D_{I_{p}}(N, E(A / \mathfrak{p})) \longrightarrow 0
$$


Therefore, combining this short exact sequence with the calculation carried out in the previous part we get

$$
D_{I_{p}}(N, E(A / \mathfrak{p}))=\left\{\begin{array}{l}
\operatorname{Hom}_{A}(N, E(A / \mathfrak{p})), \text { if } \mathfrak{p} \notin \mathrm{V}\left(I_{p}\right) \\
0, \text { if } \mathfrak{p} \in \mathrm{V}\left(I_{p}\right)
\end{array}\right.
$$

Finally, given any $\mathfrak{m} \in \operatorname{Max}(A)$ one has, as a direct application of [8, 4.1.7] (indeed, $N$ is finitely generated and the localization map $A \longrightarrow A_{\mathfrak{m}}$ is flat), that

$$
D_{I_{p}}(N, E(A / \mathfrak{p}))_{\mathfrak{m}}=\left\{\begin{array}{l}
\operatorname{Hom}_{A_{\mathfrak{m}}}\left(N_{\mathfrak{m}}, E(A / \mathfrak{p})_{\mathfrak{m}}\right), \text { if } \mathfrak{p} \notin \mathrm{V}\left(I_{p}\right) \text { and } \mathfrak{p} \subseteq \mathfrak{m} \\
0, \text { otherwise. }
\end{array}\right.
$$

Therefore, we obtain the following spectral sequence:

$$
E_{2}^{-i, j}=\mathbb{L}_{i} \operatorname{colim}_{p \in P} \mathbb{R}^{j} D_{[*]}(N, M) \Longrightarrow \mathbb{R}^{j-i} D_{I}(N, M)
$$

Notice that, when $N=A$,

$$
E_{2}^{-i, j}=\mathbb{L}_{i} \operatorname{colim}_{p \in P} \mathbb{R}^{j} D_{[*]}(M) \Longrightarrow \mathbb{R}^{j-i} D_{I}(M)
$$

In fact, this spectral sequence for ordinary Nagata's ideal transforms may be regarded as the module version of the one obtained in $[13,3.5]$. On the other hand, this spectral sequence is very closely related with the previous Mayer-Vietoris spectral sequence for local cohomology modules because of the well-known isomorphism $\mathbb{R}^{j} D_{J}(M) \cong H_{J}^{j+1}(M)$ for any $j \geq 1$ and for any ideal $J$ of $A[8,2.2 .6]$. Finally, note that, when $n=2$, we recover the long exact sequence

$$
\begin{aligned}
& 0 \longrightarrow D_{I_{1}+I_{2}}(M) \longrightarrow D_{I_{1}}(M) \oplus D_{I_{2}}(M) \longrightarrow D_{I}(M) \longrightarrow H_{I_{1}+I_{2}}^{2}(M) \\
& \ldots \longrightarrow H_{I_{1}}^{j}(M) \oplus H_{I_{2}}^{j}(M) \longrightarrow H_{I}^{j}(M) \longrightarrow H_{I_{1}+I_{2}}^{j+1}(M) \longrightarrow \ldots
\end{aligned}
$$

given in $[8,3.2 .5]$.

- Local cohomology with respect to pairs of ideals: Let $J$ be an arbitrary ideal of $A$. The direct system of torsion functors with respect to pairs of ideals $T_{[*]}=\Gamma_{[*], J}$ is given by $T_{p}(M):=\Gamma_{I_{p}, J}(M)$, that is, the $\left(I_{p}, J\right)$-torsion module with respect to $M$. The reader should remember that, for any ideal $K$ of $A$, the torsion functor $\Gamma_{K, J}$ is defined in 
the following manner:

$$
\Gamma_{K, J}(M):=\left\{m \in M \mid \quad K^{l} m \subseteq J m \text { for some } l \in \mathbb{N}\right\}
$$

Furthermore, it is known $[61,1.11]$ that $T_{[*]}$ verifies the previous requirements. In particular, $T=\Gamma_{I, J}$. Moreover, using $[61,1.11]$ once again, one deduces that

$$
\Gamma_{I_{p}, J}(E(A / \mathfrak{p}))=\left\{\begin{array}{l}
E(A / \mathfrak{p}), \text { if } \mathfrak{p} \in \mathrm{W}\left(I_{p^{\prime}} J\right), \\
0, \text { if } \mathfrak{p} \notin \mathbf{W}\left(I_{p^{\prime}} J\right) .
\end{array}\right.
$$

Here, $\mathbf{W}\left(I_{p}, J\right):=\left\{\mathfrak{p} \in \operatorname{Spec}(A) \mid I_{p}^{n} \subseteq \mathfrak{p}+J\right.$ for some integer $\left.n \geq 1\right\}$.

Therefore, we obtain the following spectral sequence:

$$
E_{2}^{-i, j}=\mathbb{L}_{i} \operatorname{colim}_{p \in P} H_{[*], J}^{j}(M) \Longrightarrow H_{I, J}^{j-i}(M) .
$$

It turns out that, when $n=2$, this spectral sequence degenerates into the following long exact sequence:

$$
\ldots \longrightarrow H_{I_{1}, J}^{j}(M) \oplus H_{I_{2}, J}^{j}(M) \longrightarrow H_{I, J}^{j}(M) \longrightarrow H_{I_{1}+I_{2}, J}^{j+1}(M) \longrightarrow \ldots
$$

This long exact sequence may be regarded as a Mayer-Vietoris long exact sequence for local cohomology modules with respect to pairs of ideals; it is worth mentioning that, to the best of our knowledge, this is the 1st time that this long exact sequence appears in the literature.

\subsection{Enhanced structure}

The main purpose now is to show, roughly speaking, that if $M$ is not only an $A$ module, but also has a certain additional structure, then the spectral sequence produced in Theorem 4.6 also inherits this structure. Throughout this part, in addition to the assumptions and notations we establish in Setup 4.3, we also require the following:

Assumption 4.7. Let $\mathcal{S} \subseteq \mathcal{A}$ be an abelian subcategory closed under subobjects, subquotients, and extensions such that, for any object $N \in \mathcal{S}$ and for any $p \in \widehat{P}$, $T_{p}(N) \in \mathcal{S}$; moreover, if $M \in \mathcal{S}$ then we suppose that there is a long exact sequence $0 \longrightarrow M \longrightarrow E^{*}$ in $\mathcal{A}$ such that

(i) For any $p \in \widehat{P}$ and for any $j \geq 0, \mathbb{R}^{k} T_{p}\left(E^{j}\right)=0$ for all $k \geq 1$.

(ii) The long exact sequence $0 \longrightarrow M \longrightarrow E^{*}$ may be regarded as an exact cochain complex in $\mathcal{S}$. 
(iii) If $A$ contains a field $\mathbb{K}$, then we also assume that, for any $M \in \mathcal{S}, \mathbb{K} \otimes_{A} M$ is also an object of $\mathcal{S}$ such that the map $\mathbb{K} \otimes_{A} M \longrightarrow M$, given by the assignment $r \otimes m \longmapsto r m$, is a natural isomorphism in $\mathcal{S}$.

We are now in a position to establish the main result of this part; namely, the following:

Theorem 4.8. Under the assumptions of Setup 4.3, if parts (i) and (ii) of Assumption 4.7 are satisfied, then one has that the spectral sequence $E_{2}^{-i, j}=\mathbb{L}_{i} \operatorname{colim}_{p \in P} \mathbb{R}^{j} T_{[*]}(M) \Longrightarrow$ $\mathbb{R}^{j-i} T(M)$ can be regarded as spectral sequence in the category $\mathcal{S}$.

Proof. Let $0 \longrightarrow M \longrightarrow E^{*}$ be the long exact sequence given by Assumption 4.7; along the proof of Theorem 4.6 we already saw that the $E_{0}$-page of our spectral sequence is given by the double complex $\operatorname{Roos}_{i}\left(T_{[*]}\left(E^{j}\right)\right)$, which again using Assumption 4.7 can be regarded as a double complex in $\mathcal{S}$. In this way, since all the pages of our spectral sequence are given by subquotients of our starting double complex, and $\mathcal{S}$ is closed under subquotients, it follows that $E_{2}^{-i, j}=\mathbb{L}_{i} \operatorname{colim}_{p \in P} \mathbb{R}^{j} T_{[*]}(M) \Longrightarrow \mathbb{R}^{j-i} T(M)$ can be regarded as spectral sequence in the category $\mathcal{S}$, as claimed.

Now, we want to introduce some specific examples where the assumptions of 4.7 are satisfied; in all these examples, unless otherwise is specified, $T_{[*]}$ is either the torsion functor $\Gamma_{[*]}$ or the ideal transform functor $D_{[*]}$.

- F-modules: Let $A$ be a commutative regular ring containing a field of prime characteristic $p$; it was shown in [36, $\left.\left(1.2 \mathrm{~b}^{\prime \prime}\right)\right]$ that, if $M$ is an $F$-module, and $0 \longrightarrow$ $M \longrightarrow E^{*}$ is its minimal injective resolution in $\mathcal{A}$, then $0 \longrightarrow M \longrightarrow E^{*}$ can also be regarded as an exact cochain complex in the category of $F$-modules. On the other hand, any injective $A$-module is $T_{p}$-acyclic for any $p \in \widehat{P}$; moreover, if $N$ is any $F$-module, then, for any $p \in \widehat{P}, T_{p}(N)$ is so. Summing up, if $\mathcal{S}$ is the category of $F$-modules, then parts (i) and (ii) of Assumption 4.7 hold; this fact was already pointed out in [4, 1.2(iii)]. Finally, notice that one has to require that $\mathbb{K}=\mathbb{F}_{p}$ in order to guarantee that part (iii) also holds; indeed, this is because, given an $F$-module $M$ with structural isomorphism $\phi$, if one wants to put an $F$-module structure on $\mathbb{K} \otimes_{A} M$ compatible with the natural isomorphism $\mathbb{K} \otimes_{A} M \longrightarrow M$, then one way to do so is to require that the action of $\phi$ on $\mathbb{K}$ is trivial, and this is the case when $\mathbb{K}=\mathbb{F}_{p}$.

- Holonomic D-modules: Let $\mathbb{K}$ be a field of characteristic 0 , let $A$ be a commutative Noetherian regular ring containing $\mathbb{K}$, let $D=D_{A \mid \mathbb{K}}$ be the ring of $\mathbb{K}$-linear 
differential operators on $A$, let $\mathcal{S}$ be the category of holonomic (left) $D$-modules, and let $M$ be a (left) holonomic $D$-module. Since the category of left $D$-modules has enough injectives, we can pick a injective resolution $0 \longrightarrow M \longrightarrow E^{*}$ of $M$ in $\mathcal{S}$; moreover, since $D$ is a free, right $A$-module it follows either from [44, Corollary 1.1 (2)] or from [52, Theorem 1 and Example 1] that $0 \longrightarrow M \longrightarrow E^{*}$ can also be regarded as an injective resolution of $A$-modules, which implies that for any $p \in \widehat{P}$ and for any $j \geq 0$, $\mathbb{R}^{k} T_{p}\left(E^{j}\right)=0$ for all $k \geq 1$. Finally, it is also known that, for any $p \in \widehat{P}$ and for any holonomic $D$-module $N, T_{p}(N)$ is also a holonomic $D$-module; in this way, $\mathcal{S}$ satisfies parts (i) and (ii) of the assumptions required in 4.7. This fact was already pointed out in [4, 1.2(iii)]. In particular, it recovers and extends [52, 3.3]. Finally, notice that part (iii) also holds mainly because the action on any $\mathbb{K}$-linear differential operator on $\mathbb{K}$ is trivial (see [14, Chapter 14 and Corollary 3.5]; in the notation there one has to consider the projection on to a point to obtain the result).

- Quasi-holonomic D-modules: Let $\mathbb{K}$ be a field of characteristic 0 , let $A$ be a differentiable admissible $\mathbb{K}$-algebra (see [48, Hypothesis 2.3] and [43, Definition 1.2.3.6]), let $T_{[*]}$ be the torsion functor $\Gamma_{[*]}$ or the torsion functor with respect to pairs of ideals $\Gamma_{[*], J}$, let $D=D_{A \mid \mathbb{K}}$ be the ring of $\mathbb{K}$-linear differential operators on $A$, let $\mathcal{S}$ be the category of quasi-holonomic (left) $D$-modules [1, Definition 3.2], and let $M$ be a (left) quasi-holonomic $D$-module. First of all, it is known [1, Lemma 3.5] that $\mathcal{S}$ is closed under formation of submodules, quotients, and extensions. Secondly, since the category of left $D$-modules has enough injectives, we can pick $0 \longrightarrow M \longrightarrow E^{*}$ an injective resolution of $M$ in $\mathcal{S}$; moreover, since $D$ is a free, right $A$-module [1, p. 2240-1] it follows either from [44, Corollary 1.1 (2)] or from [52, Theorem 1 and Example 1] that $0 \longrightarrow M \longrightarrow E^{*}$ can also be regarded as an injective resolution of $A$-modules, which implies that for any $p \in \widehat{P}$ and for any $j \geq 0, \mathbb{R}^{k} T_{p}\left(E^{j}\right)=0$ for all $k \geq 1$. Finally, it is also known that, for any $p \in \widehat{P}$ and for any quasi-holonomic $D$-module $N, T_{p}(N)$ is also a quasi-holonomic $D$-module [1, Example 3.14]; in this way, we have already checked that $\mathcal{S}$ satisfies part (i) and (ii) of Assumption 4.7. It also satisfies part (iii) because quasi-holonomic $D$-modules are exactly the ones that can be expressed as colimit of holonomic $D$-modules [1, Corollary 3.7], tensor products commute with colimits, and that we already saw that, when $M$ is holonomic, $\mathbb{K} \otimes_{A} M$ is also holonomic.

- Graded modules: Let $A$ be $\mathbb{K}\left[x_{1}, \ldots, x_{d}\right]$ graded by $\mathbb{Z}^{n}$ (for some $n \geq 1$ ), and let $\mathcal{S}$ be the category of $\mathbb{Z}^{n}$-graded $A$-modules; since $\mathcal{S}$ is an abelian category with enough injectives $[8,13.2 .4]$, we can choose, given any graded $A$-module $M$, a resolution $0 \longrightarrow M \longrightarrow E^{*}$ by injective objects of $\mathcal{S}$. Moreover, by [8, 13.2.6] one has that, for any 
injective object of $\mathcal{S}$ (namely, $E$ ), one has that $\mathbb{R}^{k} T_{p}(E)=0$ for all $p \in \widehat{P}$, and $k \geq 1$. Finally, it is also known that, for any $p \in \widehat{P}$ and for any graded $A$-module $N, T_{p}(N)$ is so. Therefore, $\mathcal{S}$ satisfies the assumptions required in Assumption 4.7 (notice that part (iii) also holds mainly because $\mathbb{K}$ is concentrated in degree zero). This fact was already pointed out in [4, Proof of 2.1].

- Modules over the group ring: Let $A$ be any commutative regular ring containing a field, let $G$ be a group, let $A[G]$ be the group ring, and let $M$ be a (left) $A[G]$-module. Since $\mathcal{S}:=A[G]-\operatorname{Mod}$ has enough injectives, we can pick $0 \longrightarrow M \longrightarrow E^{*}$ an injective resolution of $M$ in $\mathcal{S}$; moreover, since $A[G]$ is a free, right $A$-module it follows either from [44, Corollary 1.1 (2)] or from [52, Theorem 1 and Example 8] that $0 \longrightarrow M \longrightarrow E^{*}$ can also be regarded as an injective resolution of $A$-modules, which implies that for any $p \in \widehat{P}$ and for any $j \geq 0, \mathbb{R}^{k} T_{p}\left(E^{j}\right)=0$ for all $k \geq 1$. Finally, it is also known that, for any $p \in \widehat{P}$ and for any $A[G]$-module $N, T_{p}(N)$ is also an $A[G]$-module. In this way, $\mathcal{S}$ satisfies the assumptions required in Assumption 4.7 (again, notice that part (iii) also holds mainly because $\mathbb{K}$ is concentrated in degree 0 ). This fact was already shown in [37, p. 640-1] with a more direct approach.

\subsection{Degeneration of homological spectral sequences}

The goal of this subsection is to provide sufficient conditions in order to guarantee that the previously mentioned homological spectral sequences degenerate at the $E_{2}$-page. First, we shall collect some preliminary facts that will simplify the proofs of the main result of this subsection.

Definition 4.9. Let $q \in P$ and let $M$ be an object of $\mathcal{A}$. The direct system represented by $M$ on $q$ (namely, $M_{q}$ ) is defined as follows: for any $p \in P$,

$$
\left(M_{q}\right)_{p}:= \begin{cases}M, & \text { if } p=q \\ 0, & \text { otherwise }\end{cases}
$$

Next result computes the colimit of this special construction (cf. [7, 4.5 (ii)] and $[9,8.7])$.

Lemma 4.10. Let $q \in P$ and let $M$ be an object of $\mathcal{A}$. For any $i \in \mathbb{N}$,

$$
\mathbb{L}_{i} \operatorname{colim}_{p \in P}\left(M_{q}\right)_{p} \cong \widetilde{H}_{i-1}\left(\left(q, 1_{\widehat{P}}\right) ; M\right),
$$


where the tilde denotes reduced simplicial homology. We agree that the reduced homology of the empty simplicial complex is $M$ in degree -1 and 0 otherwise.

Proof. Consider the following direct systems:

$$
\left(M_{>q}\right)_{p}:=\left\{\begin{array}{l}
M, \text { if } p \in\left(q, 1_{\widehat{P}}\right), \\
0, \text { otherwise, }
\end{array} \text { and }\left(M_{\geq q}\right)_{p}:=\left\{\begin{array}{l}
M, \text { if } p \in\left[q, 1_{\widehat{P}}\right), \\
0, \text { otherwise. }
\end{array}\right.\right.
$$

Thus, we have the following short exact sequence in $\operatorname{Dir}(P, \mathcal{A})$ :

$$
0 \longrightarrow M_{>q} \longrightarrow M_{\geq q} \longrightarrow M_{q} \longrightarrow 0
$$

This leads to the following short exact sequence of chain complexes:

$$
0 \longrightarrow \operatorname{Roos}_{*}\left(M_{>q}\right) \longrightarrow \operatorname{Roos}_{*}\left(M_{\geq q}\right) \longrightarrow \operatorname{Roos}_{*}\left(M_{q}\right) \longrightarrow 0 .
$$

Moreover, this short exact sequence of complexes induces the following long exact one in homology:

$$
\ldots \rightarrow H_{i}\left(\operatorname{Roos}_{*}\left(M_{>q}\right)\right) \rightarrow H_{i}\left(\operatorname{Roos}_{*}\left(M_{\geq q}\right)\right) \rightarrow H_{i}\left(\operatorname{Roos}_{*}\left(M_{q}\right)\right) \rightarrow H_{i-1}\left(\operatorname{Roos}_{*}\left(M_{>q}\right)\right) \rightarrow \ldots
$$

On the one hand, the chain complex $\operatorname{Roos}_{*}\left(M_{\geq q}\right)$ (respectively, $\operatorname{Roos}_{*}\left(M_{>q}\right)$ ) agrees with the one for computing the reduced simplicial homology of the topological space $\left[q, 1_{\widehat{P}}\right)$ (respectively, $\left.\left(q, 1_{\widehat{P}}\right)\right)$ with coefficients on $M$; hence, one has the following natural isomorphisms:

$$
H_{i}\left(\operatorname{Roos}_{*}\left(M_{\geq q}\right)\right) \cong \widetilde{H}_{i}\left(\left[q, 1_{\widehat{P}}\right) ; M\right), H_{i}\left(\operatorname{Roos}_{*}\left(M_{>q}\right)\right) \cong \widetilde{H}_{i}\left(\left(q, 1_{\widehat{P}}\right) ; M\right) .
$$

In addition, since $\left[q, 1_{\widehat{P}}\right)$ is contractible (see Lemma 2.9$)$ it follows that $\widetilde{H}_{i}\left(\left[q, 1_{\widehat{P}}\right) ; M\right)=0$ for all $i \geq 0$; in this way, combining all the foregoing one has, for any $i \geq 1$, that there is a canonical isomorphism $H_{i}\left(\operatorname{Roos}_{*}\left(M_{\geq q}\right)\right) \cong \widetilde{H}_{i-1}\left(\left(q, 1_{\widehat{P}}\right) ; M\right)$. Finally, combining this isomorphism with the fact that the homology groups of the Roos chain complex agree with the left derived functors of the colimit (see Lemma 3.1), one finally obtains that $\mathbb{L}_{i} \operatorname{colim}_{p \in P}\left(M_{q}\right)_{p} \cong \widetilde{H}_{i-1}\left(\left(q, 1_{\widehat{P}}\right) ; M\right)$, just what we finally wanted to show. 
Now, we introduce the main result of this subsection; notice that the assumptions imposed in the following result are a slight generalization of the ones imposed in $[5,1.2]$ and $[10,1.1]$.

Theorem 4.11. Let $A$ be a commutative Noetherian ring containing a field $\mathbb{K}$, let $T_{[*]}$ be the functor introduced in Setup 4.3, and let $M$ be an object of $\mathcal{A}$ verifying the following requirements:

(a) For any $p \in P, \mathbb{R}^{j} T_{p}(M)=0$ up to a unique value of $j$ (namely, $h_{p}$ ).

(b) For any pair of distinct elements $p$ and $q$ of $P, \operatorname{Hom}_{A}\left(\mathbb{R}^{h_{p}} T_{p}(M), \mathbb{R}^{h_{q}} T_{q}(M)\right)=0$. Then, there are canonical isomorphisms of $A$-modules

$$
\mathbb{L}_{i} \operatorname{colim}_{p \in P} \mathbb{R}^{j} T_{[*]}(M) \cong \bigoplus_{j=h_{q}}\left(\widetilde{H}_{i-1}\left(\left(q, 1_{\widehat{P}}\right) ; \mathbb{K}\right) \otimes_{\mathbb{K}} \mathbb{R}^{h_{q}} T_{q}(M)\right) \cong \bigoplus_{j=h_{q}} \mathbb{R}^{h_{q}} T_{q}(M)^{\oplus m_{i, q}}
$$

(where $m_{i, q}:=\operatorname{dim}_{\mathbb{K}}\left(\widetilde{H}_{i-1}\left(\left(q, 1_{\widehat{P}}\right) ; \mathbb{K}\right)\right)$ ), and $E_{2}^{-i, j}=\mathbb{L}_{i} \operatorname{colim}_{p \in P} \mathbb{R}^{j} T_{[*]}(M) \Longrightarrow \mathbb{R}^{j-i} T(M)$ degenerates at the $E_{2}$-page.

Proof. Parts (a) and (b) of our assumptions imply that there is a canonical isomorphism of direct systems of $A$-modules

$$
\mathbb{R}^{j} T_{[*]}(M) \cong \bigoplus_{j=h_{q}}\left(\mathbb{R}^{h_{q}} T_{q}(M)\right)_{q}
$$

Indeed, the direct system $\mathbb{R}^{j} T_{[*]}(M)$ is the one given by $\left(\mathbb{R}^{j} T_{p}(M)\right)_{p \in P}=\left(\mathbb{R}^{h_{p}} T_{p}(M)\right)_{p \in P}$ $\left(j=h_{p}\right)$, where the only nonzero structural maps are identities; in this way, these facts imply that this direct system splits into the above direct sum.

Now, fix $i \in \mathbb{N}$. Applying to the above decomposition the $i$ th left derived functor of the colimit over $P$, we get the following canonical isomorphism of $A$-modules:

$$
\mathbb{L}_{i} \operatorname{colim}_{p \in P} \mathbb{R}^{j} T_{[*]}(M) \cong \bigoplus_{j=h_{q}} \mathbb{L}_{i} \operatorname{colim}_{p \in P}\left(\mathbb{R}^{h_{q}} T_{q}(M)\right)_{q}
$$

Moreover, Lemma 4.10 implies that there is a canonical isomorphism of $A$-modules:

$$
\mathbb{L}_{i} \operatorname{colim}_{p \in P} \mathbb{R}^{j} T_{[*]}(M) \cong \bigoplus_{j=h_{q}} \widetilde{H}_{i-1}\left(\left(q, 1_{\widehat{P}}\right) ; \mathbb{R}^{h_{q}} T_{q}(M)\right)
$$


Next, since the map $\widetilde{H}_{i-1}\left(\left(q, 1_{\widehat{P}}\right) ; \mathbb{K}\right) \otimes_{\mathbb{K}} \mathbb{R}^{h_{q}} T_{q}(M) \rightarrow \widetilde{H}_{i-1}\left(\left(q, 1_{\widehat{P}}\right) ; \mathbb{R}^{h_{q}} T_{q}(M)\right)$ given by the assignment $\operatorname{cls}(z) \otimes a \longmapsto \operatorname{cls}(z \otimes a)$ is a natural isomorphism of $A$-modules (indeed, because any $\widetilde{H}_{i-1}\left(\left(q, 1_{\widehat{P}}\right) ; \mathbb{K}\right)$ is flat over the field $\left.\mathbb{K}\right)$ one obtains the below natural isomorphism of $A$-modules:

$$
\mathbb{L}_{i} \operatorname{colim}_{p \in P} \mathbb{R}^{j} T_{[*]}(M) \cong \bigoplus_{j=h_{q}}\left(\widetilde{H}_{i-1}\left(\left(q, 1_{\widehat{P}}\right) ; \mathbb{K}\right) \otimes_{\mathbb{K}} \mathbb{R}^{h_{q}} T_{q}(M)\right) .
$$

Now, set $m_{i, q}:=\operatorname{dim}_{\mathbb{K}}\left(\widetilde{H}_{i-1}\left(\left(q, 1_{\widehat{P}}\right) ; \mathbb{K}\right)\right)$, so $\widetilde{H}_{i-1}\left(\left(q, 1_{\widehat{P}}\right) ; \mathbb{K}\right) \cong \mathbb{K}^{\oplus m_{i, q}}$. On the other hand, the natural map $\mathbb{K} \otimes_{\mathbb{K}} \mathbb{R}^{h_{q}} T_{q}(M) \longrightarrow \mathbb{R}^{h_{q}} T_{q}(M)$ given by the assignment $r \otimes x \longmapsto r x$ is a natural isomorphism of $A$-modules obtained by extending scalars [56, Propositions 2.51 and 2.58], and since tensor product preserves direct sums [56, Theorem 2.65], one obtains a natural isomorphism $\widetilde{H}_{i-1}\left(\left(q, 1_{\widehat{P}}\right) ; \mathbb{K}\right) \otimes_{\mathbb{K}} \mathbb{R}^{h_{q}} T_{q}(M) \cong \mathbb{R}^{h_{q}} T_{q}(M)^{\oplus m_{i, q}}$ of $A$ modules, and therefore we finally have a natural isomorphism

$$
\bigoplus_{j=h_{q}}\left(\widetilde{H}_{i-1}\left(\left(q, 1_{\widehat{P}}\right) ; \mathbb{K}\right) \otimes_{\mathbb{K}} \mathbb{R}^{h_{q}} T_{q}(M)\right) \cong \bigoplus_{j=h_{q}} \mathbb{R}^{h_{q}} T_{q}(M)^{\oplus m_{i, q}}
$$

of $A$-modules. In this way, combining the previous isomorphism joint with part (b) of our assumptions one obtains the announced degeneration.

When a spectral sequence degenerates at the $E_{2}$-page, it is natural to consider the corresponding filtration that this degeneration provides. This is the content of the following direct consequence of Theorem 4.11 .

Corollary 4.12. Let $A$ be a commutative Noetherian ring containing a field $\mathbb{K}$, let $T_{[*]}$ be the functor introduced in Section 4 , and let $M$ be an object of $\mathcal{A}$ verifying the following requirements:

(a) For any $p \in P, \mathbb{R}^{j} T_{p}(M)=0$ up to a unique value of $j$ (namely, $h_{p}$ ).

(b) For any pair of distinct elements $p$ and $q$ of $P, \operatorname{Hom}_{A}\left(\mathbb{R}^{h_{p}} T_{p}(M), \mathbb{R}^{h_{q}} T_{q}(M)\right)=0$. Then, for each $0 \leq r \leq \operatorname{cd}(T)$ (see Setup 4.3 for the definition of $\operatorname{cd}(T)$ ) there is an increasing, finite filtration $\left\{G_{k}^{r}\right\}$ of $\mathbb{R}^{r} T(M)$ by $A$-modules such that, for any $k \geq 0$,

$$
G_{k}^{r} / G_{k-1}^{r} \cong \bigoplus_{\left\{q \in P \mid k+r=h_{q}\right\}} \mathbb{R}^{h_{q}} T_{q}(M)^{\oplus m_{k, q}}
$$

where $m_{k, q}:=\operatorname{dim}_{\mathbb{K}}\left(\widetilde{H}_{k-1}\left(\left(q, 1_{\widehat{P}}\right) ; \mathbb{K}\right)\right)$ and we follow the convention that $G_{-1}^{r}=0$. 
Proof. Under these assumptions, Theorem 4.11 ensures that the spectral sequence

$$
E_{2}^{-i, j}=\mathbb{L}_{i} \operatorname{colim}_{p \in P} \mathbb{R}^{j} T_{[*]}(M) \underset{i}{\Longrightarrow} \mathbb{R}^{j-i} T(M)
$$

degenerates at the $E_{2}$-page; moreover, Theorem 4.11 says that

$$
E_{2}^{-i, j}=\bigoplus_{\left\{q \in P \mid j=h_{q}\right\}} \mathbb{R}^{h_{q}} T_{q}(M)^{\oplus m_{i, q}}
$$

Now, fix $0 \leq r \leq \operatorname{cd}(T)$. From the degeneration at the $E_{2}$-page obtained in Theorem 4.11 and the very definition of convergence of a spectral sequence [56, pp. 626-7], one gets an increasing finite filtration $\left\{G_{k}^{r}\right\}$ of $\mathbb{R}^{r} T(M)$ by $A$-modules such that $G_{k}^{r} / G_{k-1}^{r} \cong E_{2}^{-k, k+r}$. Therefore, we get

$$
G_{k}^{r} / G_{k-1}^{r} \cong \bigoplus_{\left\{q \in P \mid k+r=h_{q}\right\}} \mathbb{R}^{h_{q}} T_{q}(M)^{\oplus m_{k, q}}
$$

just what we finally wanted to show.

We also write down the enriched version (see Assumption 4.7) of Corollary 4.12, which can be proved using the same kind of arguments involved in the proof of Theorem 4.8 and Corollary 4.12 .

Corollary 4.13. Let $A$ be a commutative Noetherian ring containing a field $\mathbb{K}$, let $T_{[*]}$ be the functor introduced in Section 4 , let $\mathcal{S}$ be a subcategory of $\mathcal{A}$ satisfying Assumption 4.7, and let $M$ be an object of $\mathcal{S}$ verifying the following requirements:

(a) For any $p \in P, \mathbb{R}^{j} T_{p}(M)=0$ up to a unique value of $j$ (namely, $h_{p}$ ).

(b) For any pair of distinct elements $p$ and $q$ of $P, \operatorname{Hom}_{\mathcal{S}}\left(\mathbb{R}^{h_{p}} T_{p}(M), \mathbb{R}^{h_{q}} T_{q}(M)\right)=0$. Then, for each $0 \leq r \leq \operatorname{cd}(T)$ there is an increasing, finite filtration $\left\{G_{k}^{r}\right\}$ of $\mathbb{R}^{r} T(M)$ by objects of $\mathcal{S}$ such that, for any $k \geq 0$,

$$
G_{k}^{r} / G_{k-1}^{r} \cong \bigoplus_{\left\{q \in P \mid k+r=h_{q}\right\}} \mathbb{R}^{h_{q}} T_{q}(M)^{\oplus m_{k, q}}
$$

(where $m_{k, q}:=\operatorname{dim}_{\mathbb{K}}\left(\widetilde{H}_{k-1}\left(\left(q, 1_{\widehat{P}}\right) ; \mathbb{K}\right)\right)$ and we follow the convention that $G_{-1}^{r}=0$ ), and all these isomorphisms are isomorphisms in the category $\mathcal{S}$. 


\subsubsection{Examples of degeneration}

The goal of this part is to specialize Theorem 4.11 (and, in particular, Corollary 4.12) to several specific choices of $T_{[*]}$ and $P$.

Keeping this aim in mind, we review for the convenience of the reader the following:

Definition 4.14. Let $A$ be a commutative Noetherian ring, and let $I \subseteq A$ be an ideal; it is said that $I$ is cohomologically complete intersection provided $H_{I}^{k}(A) \neq 0$ for any $k \neq \mathrm{ht}(I)$.

Cohomologically complete intersection ideals were introduced by Hellus and Schenzel in [25], where the interested reader on this notion can find further details and results. This situation is achieved, among others, in the following cases:

- $A$ is a regular ring containing a field of prime characteristic, which is either local or a polynomial ring over that field, and $I \subseteq A$ is an ideal (homogeneous in the polynomial ring case) such that $A / I$ is Cohen-Macaulay [51, Chapter IV, Proposition 4.1, and Corollary 4.2].

- $A$ is either a polynomial ring or a formal power series ring over a field of arbitrary characteristic, $A / I$ is Cohen-Macaulay, and $I$ is a squarefree monomial ideal [34, Theorem 1 (iii)].

When $A / I$ is Cohen-Macaulay containing a field of characteristic 0 , it is no longer true that $I$ is a cohomologically complete intersection ideal; take, for instance, $A$ as the polynomial ring in six variables over any field of characteristic 0 , and take $I$ as the ideal generated by the $2 \times 2$ minors of a generic $2 \times 3$ matrix [29, Example 21.31].

We plan to use the following property of this kind of ideals.

Lemma 4.15. Let $A$ be a commutative Noetherian Gorenstein ring, and let $\mathfrak{p}$ and $\mathfrak{q}$ be prime ideals of $A$; assume, in addition, that $\mathfrak{p} \nsubseteq \mathfrak{q}$, that $\mathrm{ht}(\mathfrak{p}) \neq \mathrm{ht}(\mathfrak{q})$, and that both $\mathfrak{p}$ and $\mathfrak{q}$ are cohomologically complete intersection ideals. Then, one has that $\operatorname{Hom}_{A}\left(H_{\mathfrak{p}}^{\mathrm{ht}(\mathfrak{p})}(A), H_{\mathfrak{q}}^{\mathrm{ht}(\mathfrak{q})}(A)\right)=0$.

Proof. For the sake of brevity, set $h_{p}:=\operatorname{ht}(\mathfrak{p})$ and $h_{q}:=\mathrm{ht}(\mathfrak{q})$. So, since $\mathfrak{p} \nsubseteq \mathfrak{q}$ and $\mathfrak{q}$ is prime, $(\mathfrak{q}: \mathfrak{p})=\mathfrak{q}$, and therefore

$$
\operatorname{Hom}_{A}(A / \mathfrak{p}, A / \mathfrak{q})=\frac{(\mathfrak{q}: \mathfrak{p})}{\mathfrak{q}}=0 ;
$$

this is the basic fact we are going to use very soon. 
Assume, to get a contradiction, that there is $0 \neq \psi \in \operatorname{Hom}_{A}\left(H_{\mathfrak{p}}^{h_{p}}(A), H_{\mathfrak{q}}^{h_{q}}(A)\right)$; since $A$ is Gorenstein, $A$ admits a minimal injective resolution of the form

$$
0 \longrightarrow A \stackrel{d^{-1}}{\longrightarrow} E^{0} \stackrel{d^{0}}{\longrightarrow} E^{1} \longrightarrow \ldots \longrightarrow E^{n-1} \stackrel{d^{n-1}}{\longrightarrow} E^{n} \stackrel{d^{n}}{\longrightarrow} 0,
$$

where $n=\operatorname{dim}(A)$ and, for any $0 \leq k \leq n$,

$$
E^{k}=\bigoplus_{\left\{\mathfrak{p}^{\prime} \in \operatorname{Spec}(A): \operatorname{ht}\left(\mathfrak{p}^{\prime}\right)=k\right\}} E\left(A / \mathfrak{p}^{\prime}\right) .
$$

This implies that $\Gamma_{\mathfrak{p}}\left(E^{h_{p}-1}\right)=0$ and $\Gamma_{\mathfrak{q}}\left(E^{h_{q}-1}\right)=0$; hence,

$$
H_{\mathfrak{p}}^{h_{p}}(A)=\frac{\operatorname{ker}\left(\Gamma_{\mathfrak{p}}\left(d^{h^{p}}\right)\right)}{\operatorname{Im}\left(\Gamma_{\mathfrak{p}}\left(d^{h^{p}-1}\right)\right)} \subseteq E(A / \mathfrak{p})
$$

and

$$
H_{\mathfrak{q}}^{h_{q}}(A)=\frac{\operatorname{ker}\left(\Gamma_{\mathfrak{q}}\left(d^{h^{q}}\right)\right)}{\operatorname{Im}\left(\Gamma_{\mathfrak{q}}\left(d^{h^{q}-1}\right)\right)} \subseteq E(A / \mathfrak{q})
$$

These inclusions, combined with the fact that $\operatorname{Ass}_{A}(E(A / \mathfrak{p}))=\{\mathfrak{p}\}$ (respectively, $\left.\operatorname{Ass}_{A}(E(A / \mathfrak{q}))=\{\mathfrak{q}\}\right)$, imply that $\operatorname{Ass}_{A}\left(H_{\mathfrak{p}}^{h_{p}}(A)\right)=\{\mathfrak{p}\}$ and $\operatorname{Ass}_{A}\left(H_{\mathfrak{q}}^{h_{q}}(A)\right)=\{\mathfrak{q}\}$. Therefore, one has that, if $H_{\mathfrak{p}}^{h_{p}}(A) \stackrel{\psi}{\longrightarrow} H_{\mathfrak{q}}^{h_{q}}(A)$ is nonzero, then there is a nonzero $A$-linear map $A / \mathfrak{p} \longrightarrow$ $A / \mathfrak{q}$; but this is impossible because we have already seen that $\operatorname{Hom}_{A}(A / \mathfrak{p}, A / \mathfrak{q})=0$.

Remark 4.16. Notice that the assumption $h_{p} \neq h_{q}$ in Lemma 4.15 cannot be dropped, in general; indeed, suppose that, for instance, $h:=h_{p}=h_{q}$ and that $\mathfrak{q} \subseteq \mathfrak{p}$, so we can write $\mathfrak{p}=\mathfrak{q}+J$ for some ideal $J \subseteq A$. In this case, the Mayer-Vietoris long exact sequence produces the following natural map: $H_{\mathfrak{p}}^{h}(A) \longrightarrow H_{\mathfrak{q}}^{h}(A) \oplus H_{J}^{h}(A)$. If we compose this map with the projection on to the 1st factor of its target, then one obtains a potentially nonzero homomorphism $H_{\mathfrak{p}}^{h}(A) \longrightarrow H_{\mathfrak{q}}^{h}(A)$.

As a direct consequence of Corollary 4.12 and Lemma 4.15, we obtain the following:

Theorem 4.17. Let $A$ be a commutative Noetherian Gorenstein ring containing a field $\mathbb{K}$, and let $I \subseteq A$ be an ideal such that the poset $P$ is made up by cohomologically complete intersection ideals; moreover, suppose that, for any $p<q, h_{q}<h_{p}$. Then, for each $0 \leq r \leq \operatorname{dim}(A)$ there is an increasing, finite filtration $\left\{G_{k}^{r}\right\}$ of $H_{I}^{r}(A)$ by $A$-modules such 
that, for any $k \geq 0$,

$$
G_{k}^{r} / G_{k-1}^{r} \cong \bigoplus_{\left\{q \in P \mid k+r=h_{q}\right\}} H_{I_{q}}^{\mathrm{ht}\left(I_{q}\right)}(A)^{\oplus m_{k, q}}
$$

where $m_{k, q}:=\operatorname{dim}_{\mathbb{K}}\left(\widetilde{H}_{k-1}\left(\left(q, 1_{\widehat{P}}\right) ; \mathbb{K}\right)\right)$ and we follow the convention that $G_{-1}^{r}=0$.

Now we specialize the conclusion of Theorem 4.17 to some specific examples.

- Linear arrangements: Let $\mathbb{K}$ be a field, and let $I \subseteq \mathbb{K}\left[x_{1}, \ldots, x_{d}\right]=: A$ be the defining ideal of an arrangement of linear varieties of $\mathbb{K}^{d}$; it is known that $I$ admits a minimal primary decomposition of the form $I=I_{1} \cap \ldots \cap I_{n}$, where any $I_{j}$ is a prime ideal of $A$ generated by polynomials of total degree at most 1 . It is also clear that any sum of the $I_{k}$ s gives another prime ideal of the same kind, so the poset $P$ is made up by prime ideals of this form, in particular all of them are cohomologically complete intersection ideals; so, the assumptions of Theorem 4.17 are satisfied in this case. This example is the one already studied in detail in [5].

- Toric face ideals in prime characteristic: Let $\Sigma \subseteq \mathbb{R}^{d}$ be a rational pointed fan, let $\mathcal{M}_{\Sigma}$ be a monoidal complex supported on $\Sigma$, let $\mathbb{K}$ be a field, and let $\mathbb{K}\left[\mathcal{M}_{\Sigma}\right]$ be the corresponding toric face ring. It is known $\left[46\right.$, p. 539] that $\mathbb{K}\left[\mathcal{M}_{\Sigma}\right] \cong \mathbb{K}\left[x_{1}, \ldots, x_{d}\right] / I$, where $I$ is generated by squarefree monomials and pure binomials; we refer to any $I$ of this type as a toric face ideal. Moreover, it is also known that $I$ admits a minimal primary decomposition

$$
I=\mathfrak{p}_{C_{1}} \cap \ldots \cap \mathfrak{p}_{C_{t}}
$$

where $\mathfrak{p}_{C_{i}}$ is the $\mathbb{Z}^{d}$-graded prime ideal of $A:=\mathbb{K}\left[x_{1}, \ldots, x_{d}\right]$ corresponding to one of the maximal cones of $\Sigma$ (namely, $C_{i}$ ); in addition, since $\mathfrak{p}_{C_{i}}+\mathfrak{p}_{C_{j}}=\mathfrak{p}_{C_{i} \cap C_{j}}$ [28, Lemma 2.3 (i)] one can ensure that the poset $P$ is made up by prime ideals of $A$.

Now, assume that $\mathbb{K}$ is a field of prime characteristic $p$ and $\mathcal{M}_{\Sigma}$ is a CohenMacaulay monoidal complex; this last condition means that, for any cone $C$ of $\Sigma, A / \mathfrak{p}_{C}$ is a Cohen-Macaulay ring. Under these assumptions, for any cone $C$ of $\Sigma, \mathfrak{p}_{C}$ is a cohomologically complete intersection prime ideal.

Summing up, we have seen that, if $I \subseteq \mathbb{K}\left[x_{1}, \ldots, x_{d}\right]$ is the defining ideal of a toric face ring of the form $\mathbb{K}\left[\mathcal{M}_{\Sigma}\right]$, where $\mathbb{K}$ is a field of prime characteristic $p$ and $\mathcal{M}_{\Sigma}$ is a Cohen-Macaulay monoidal complex, then the poset $P$ is made up by cohomologically complete intersection prime ideals, which is what we need to prove. 
Remark 4.18. We want to single out here that, from Theorem 4.17, one can immediately deduce a Mustaţâ-Terai-type formula for certain toric face rings; we plan to write down this formula later on in this paper (see Theorem 7.14).

\subsection{Additive functions}

Our next goal is to exploit the filtration obtained in Corollary 4.13 to extend the formula of characteristic cycles of local cohomology modules given in [4, 1.3]. Actually, the most general statement we obtain, which is a direct consequence of Corollary 4.13, is the following:

Theorem 4.19. Under the assumptions of Corollary 4.13, let $\mathcal{S}$ be as in Assumption 4.7, let $G$ be an abelian group, and let $\mathcal{S} \stackrel{\lambda}{\longrightarrow} G$ be an additive function. If $M \in \mathcal{S}$ then, for any $0 \leq r \leq \operatorname{cd}(T)$,

$$
\lambda\left(\mathbb{R}^{r} T(M)\right)=\sum_{p \in P} m_{r, p} \lambda\left(\mathbb{R}^{h_{p}} T_{p}(M)\right)
$$

where $m_{r, p}=\operatorname{dim} \mathbb{K}_{h_{p}-r-1}\left(\left(p, 1_{\widehat{P}}\right) ; \mathbb{K}\right)$.

Now, we want to specialize the above formula for several specific choices of $\lambda$.

- Characteristic cycles: Let $\mathbb{K}$ be a field of characteristic 0 , let $\mathcal{S}$ be the category of holonomic $D$-modules on $A$ (where $A$ is a commutative Noetherian regular ring containing $\mathbb{K}$ ), and let $T_{[*]}$ be $\Gamma_{[*]}$. Since the characteristic cycle CC is additive, we obtain the following formula:

$$
\mathrm{CC}\left(H_{I}^{r}(A)\right)=\sum_{p \in P} m_{r, p} T_{X_{p}}^{*} \mathbb{K}^{d}
$$

where $T_{X_{p}}^{*} \mathbb{K}^{d}$ denotes the relative conormal subspace of $T^{*} \mathbb{K}^{d}$ attached to $X_{p}=\mathrm{V}\left(I_{p}\right)$. As the reader can easily point out, this formula recovers and extends the one given in $[5,1.3]$.

- Length of a D-module: Let $A$ be a commutative Noetherian regular ring containing a field $\mathbb{K}$, let $D=D_{A \mid \mathbb{K}}$ be the ring of $\mathbb{K}$-linear differential operators on $A$, let $\mathcal{S}$ be the category of left $D$-modules, and let $\ell_{D}$ be the length function of this category; since $\ell_{D}$ is additive, Theorem 4.19 ensures that, for any $D$-module $M$ for which the assumptions of Corollary 4.12 are satisfied,

$$
\ell_{D}\left(H_{I}^{r}(M)\right)=\sum_{p \in P} m_{r, p} \ell_{D}\left(H_{I_{p}}^{h_{p}}(M)\right)
$$


The reader will easily note that this formula provides an equality of generalized Lyubeznik numbers when $M=A$ [49, Definition 4.3].

- Length of an F-module: Let $A$ be a commutative Noetherian regular ring containing a field of prime characteristic $p$, let $\mathcal{S}$ be the category of $F$-modules, and let $\ell_{F}$ be the length function of this category; since $\ell_{F}$ is additive, Theorem 4.19 ensures that, for any $F$-module $M$ for which the assumptions of Corollary 4.12 are satisfied,

$$
\ell_{F}\left(H_{I}^{r}(M)\right)=\sum_{p \in P} m_{r, p} \ell_{F}\left(H_{I_{p}}^{h_{p}}(M)\right)
$$

In the case $A=\mathbb{F}_{p} \llbracket x_{1}, \ldots, x_{d} \rrbracket$ this equality is equivalent, by the definition of Lyubeznik's quasi length [36, Theorem 4.5], to the one below:

$$
\mathrm{ql}\left(H_{\mathfrak{m}}^{d-r}(A / I)\right)=\sum_{p \in P} m_{r, p} \mathrm{ql}\left(H_{\mathfrak{m}}^{\operatorname{dim}\left(A / I_{p}\right)}\left(A / I_{p}\right)\right)
$$

On the other hand, if $A=\mathbb{F}_{p}\left[x_{1}, \ldots, x_{n}\right]$, then the above equality provides a closed formula for the calculation of $\ell_{F}\left(H_{I}^{r}(A)\right)$; it is worth noting that, in [31, Corollary 3.6], they provide the following upper bound for the length:

$$
\ell_{F}\left(H_{I}^{r}(A)\right) \leq \sum_{1 \leq i_{1} \leq \ldots \leq i_{j} \leq t}\left(\left(d_{i_{1}}+\ldots+d_{i_{j}}+1\right)^{n}-1\right)
$$

where $I$ is generated by polynomials $f_{1}, \ldots, f_{t}$ with $\operatorname{deg}\left(f_{k}\right)=d_{k}$.

\section{Cohomological Spectral Sequences Associated to Inverse Systems}

As in the homological setup, the goal of this section is to build some spectral sequences, in spite of the fact that we are mostly interested on those involving local cohomology modules; actually, the spectral sequences we are going to build involve in their 2nd page the right derived functors of the limit, for this reason we refer to them as cohomological.

Let $A$ be a commutative Noetherian $\operatorname{ring} A$ and $\mathcal{A}$ the category of $A$-modules. In this section we will either consider the poset associated to an ideal $I \subseteq A$ given in Example 2.1 or the poset associated to a rational pointed fan $\Sigma \subseteq \mathbb{R}^{d}$ given in Example 2.2. The main difference with respect to the construction of spectral sequences given in Section 4 is that our starting point is going to be an inverse system of $A$-modules over a poset instead of just an $A$-module. Most commonly, given an ideal $I \subseteq A$ and an 
$A$-module $M$, we will consider the inverse system $M /[*] M:=\left(M / I_{p} M\right)_{p \in P}$ or given a rational pointed fan $\Sigma \subseteq \mathbb{R}^{d}$ we will consider the inverse system $\left(\mathbb{K}\left[\mathcal{M}_{\Sigma_{p}}\right]\right)_{p \in P}$.

Before going on we should point out that, in general, it is not true that, neither $M /[*] M$ is acyclic with respect to the limit, nor the isomorphism $M / I M \cong \lim _{p \in P} M / I_{p} M$. In the case that $M=A$ some sufficient conditions to guarantee this isomorphism were given in [9, Example 3.3].

Proposition 5.1. Assume that $P$ is a subset of a distributive lattice of ideals of $A$ (with respect to sum and intersection) or, more generally, that $A /[*]$ is acyclic with respect to the limit and the natural homomorphism $A \longrightarrow \lim _{p \in P} A / I_{p}$ is surjective. Then, there is a canonical isomorphism $A / I \cong \lim _{p \in P} A / I_{p}$.

For the case of monomial ideals these conditions are satisfied [10, Example 3.3]. For toric face ideals this is also true [12, Proposition 2.2 and Proof], so we have an isomorphism $\mathbb{K}\left[\mathcal{M}_{\Sigma}\right] \cong \lim _{p \in P} \mathbb{K}\left[\mathcal{M}_{\Sigma_{p}}\right]$.

However, as we already observed, it is in general not true that $M / I M$ is isomorphic to $\lim _{p \in P} M / I_{p} M$, even in the case where $M=A$. We specially thank Jack Jeffries for pointing out to us the following:

Remark 5.2. The sufficient conditions given in Proposition 5.1 are, in general, not satisfied for ideals defining arrangements of linear varieties; more precisely, in what follows we give an example where $A / I$ is not isomorphic to $\lim _{p \in P} A / I_{p}$. Indeed, for instance take the ideal $I=(x) \cap(y) \cap(x-y) \subseteq \mathbb{K}[x, y]=A$, where $\mathbb{K}$ is any field. In this case, the poset $P$ is

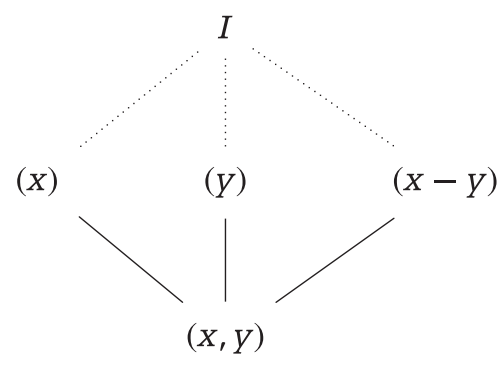

It is straightforward to check that

$$
((x)+(y)) \cap(x-y)=(x-y) \supsetneq(x(x-y), y(x-y))=((x) \cap(x-y))+((y) \cap(x-y)),
$$

hence, $P$ cannot be a subset of a distributive lattice of ideals of $A$ with respect to sum and intersection. 
Our next goal is to show that $A / I$ cannot be isomorphic to $\lim _{p \in P} A / I_{p}$; actually, it is enough to show that the natural monomorphism $A / I \hookrightarrow \lim _{p \in P} A / I_{p}$ is not surjective. Indeed, $\lim _{p \in P} A / I_{p}$ is the kernel of the unique nonzero differential of the Roos cochain complex; namely, the following:

$$
\begin{aligned}
& A /(x) \times A /(y) \times A /(x-y) \times A /(x, y) \longrightarrow A /(x, y) \times A /(x, y) \times A /(x, y) \\
& (a, b, c, d) \longmapsto(a-d, b-d, c-d) .
\end{aligned}
$$

Consider the constant map $A / I \longrightarrow A /(x) \times A /(y) \times A /(x-y) \times A /(x, y)$ mapping $a$ to $(a, a, a, a)$; it is enough to show that this constant map is not surjective.

Indeed, suppose (to get a contradiction) that, given polynomials $a, b, c \in A$ such that (without loss of generality) $a(0,0)=b(0,0)=c(0,0)=0$, there is a polynomial $P=P(x, y)$ such that $P(0,0)=0, P(0, y)=a(0, y), P(x, 0)=b(x, 0)$, and $P(x, x)=c(x, x)$ (this is equivalent to say that the above constant map is surjective). It is straightforward to check that, under these assumptions, $\operatorname{deg}(P)$ (by $\operatorname{deg}(P)$ we mean the total degree of $P$ ) is less than or equal to $\operatorname{deg}_{y}(a)+\operatorname{deg}_{x}(b)$. Therefore, to get a contradiction, one only needs to pick $c$ such that $\operatorname{deg}(c) \geq \operatorname{deg}_{y}(a)+\operatorname{deg}_{X}(b)+1$. For a concrete example, just take $a=y, b=x$, and $c=x^{2}$.

Summing up, we have seen that our above constant map cannot be surjective; hence, $A / I$ cannot be isomorphic to $\lim _{p \in P} A / I_{p}$.

Remark 5.3. We also want to point out that, although $P$ may not be a subset of a distributive lattice of ideals of $A$, one might still have that the natural map $A \longrightarrow$ $\lim _{p \in P} A / I_{p}$ is surjective; indeed, consider $I=(x, y) \cap(x, z) \cap(x-z, y) \subseteq \mathbb{K}[x, y, z]=A$, where $\mathbb{K}$ is any field. In this case, the poset $P$ is

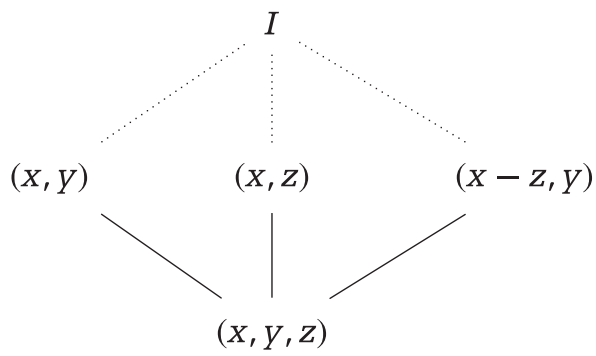

It is straightforward to check that

$$
((x, y)+(x, z)) \cap(x-z, y)=(x-z, y) \supsetneq((x, y) \cap(x-z, y))+((x, z) \cap(x-z, y)),
$$


hence, $P$ cannot be a subset of a distributive lattice of ideals of $A$ with respect to sum and intersection. However, in this case, a similar calculation to the one carried out in Remark 5.2 shows that $A \longrightarrow \lim _{p \in P} A / I_{p}$ is surjective, and therefore there is an isomorphism $A / I \cong \lim _{p \in P} A / I_{p}$.

\subsection{Construction of cohomological spectral sequences}

The reader is encouraged to compare the following setup with the ones carried out in Sections 4.3 and 6.1; indeed, in this case we are going to produce an endofunctor on inverse systems of $A$-modules carrying over a given endofunctor of $A$-modules.

Setup 5.4. Let $\mathcal{A} \stackrel{T}{\longrightarrow} \mathcal{A}$ be a covariant, left exact functor, and let $P$ be any finite poset. Building upon $T$, we produce the following endofunctor on $\operatorname{Inv}(P, \mathcal{A})$ : namely,

$$
\begin{aligned}
& \operatorname{Inv}(P, \mathcal{A}) \stackrel{\mathcal{T}}{\longrightarrow} \operatorname{Inv}(P, \mathcal{A}) \\
& M=\left(M_{p}\right)_{p \in P} \longmapsto \mathcal{T}(M):=\left(T\left(M_{p}\right)\right)_{p \in P} .
\end{aligned}
$$

In addition, we suppose that $T$ commutes with arbitrary direct sums and that there are ideals $J$ and $K$ of $A$ such that $T$ verifies one (and only one) of the following two assumptions:

(a) For any $\mathfrak{p} \in \operatorname{Spec}(A)$ and for any maximal ideal $\mathfrak{m}$ of $A$, there exists an $A$-module $X$ such that

$$
T(E(A / \mathfrak{p}))_{\mathfrak{m}}=\left\{\begin{array}{l}
X, \text { if } \mathfrak{p} \in \mathbf{W}(J, K) \text { and } \mathfrak{p} \subseteq \mathfrak{m} \\
0, \text { otherwise }
\end{array}\right.
$$

Here, $X$ depends on $J, K, T, \mathfrak{p}$, and $\mathfrak{m}$.

(b) For any $\mathfrak{p} \in \operatorname{Spec}(A)$ and for any maximal ideal $\mathfrak{m}$ of $A$, there exists an $A$-module $Y$ such that

$$
T(E(A / \mathfrak{p}))_{\mathfrak{m}}=\left\{\begin{array}{l}
Y, \text { if } \mathfrak{p} \notin \mathrm{W}(J, K) \text { and } \mathfrak{p} \subseteq \mathfrak{m} \\
0, \text { otherwise. }
\end{array}\right.
$$

Here, $Y$ depends on $J, K, T, \mathfrak{p}$, and $\mathfrak{m}$.

In what follows, for a functor $\mathcal{T}$, we denote by $\mathbb{R}^{j} \mathcal{T}$ its corresponding $j$ th right derived functor in the abelian category $\operatorname{Inv}(P, \mathcal{A})$. 


\subsubsection{Main result}

As we have previously explained, our goal is to construct a spectral sequence that involves the right derived functors of the limit. The following lemma turns out to be the 1 st step in this construction.

Lemma 5.5. Let $\Upsilon$ be an injective inverse system of the form

$$
\bigoplus_{j \in J}\left(E_{j} P\right)_{\geq q_{j}}
$$

where $J$ is a (not necessarily finite) index set, $q_{j} \in P, E_{j}$ is an indecomposable injective $A$-module, and

$$
\left[\left(E_{j} P\right)_{\geq q_{j}}\right]_{p}:=\left\{\begin{array}{l}
E_{j}, \text { if } p \in\left[q_{j}, 1_{\widehat{P}}\right), \\
0, \text { otherwise. }
\end{array}\right.
$$

Then, the coaugmented cochain complex

$$
0 \longrightarrow\left(\lim _{p \in P} \circ \mathcal{T}\right)(\Upsilon) \longrightarrow \operatorname{Roos}^{*}(\mathcal{T}(\Upsilon))
$$

is exact.

Proof. As $\mathcal{T}$ and Roos* commute with direct sums we may assume, without loss of generality, that $\Upsilon=(E(A / \mathfrak{p}) P)_{\geq q}$ for some $(\mathfrak{p}, q) \in \operatorname{Spec}(A) \times P$.

Now, we carry out a similar strategy as the one employed in the proof of Lemma 4.4; indeed, fix a maximal ideal $\mathfrak{m}$ of $A$. By the usual generalities, it is enough to show that the cochain complex

$$
0 \longrightarrow\left(\lim _{p \in P} \circ \mathcal{T}\right)(\Upsilon)_{\mathfrak{m}} \longrightarrow \operatorname{Roos}^{*}(\mathcal{T}(\Upsilon))_{\mathfrak{m}}
$$

is exact. If $\mathfrak{p} \nsubseteq \mathfrak{m}$ then the previous coaugmented cochain complex is zero and we are done; therefore, from now on we suppose that $\mathfrak{p} \subseteq \mathfrak{m}$.

First of all, suppose that $T$ verifies requirement (a) of Setup 5.4; on the one hand, if $\mathfrak{p} \notin \mathrm{W}(J, K)$, then our coaugmented cochain complex is identically zero, whence we are done. On the other hand, if $\mathfrak{p} \in \mathbf{W}(J, K)$, then this coaugmented cochain complex turns out to be equal to the one for computing the simplicial cohomology of the topological space $\left[q, 1_{\widehat{P}}\right.$ ) with coefficients in $X$ (see Lemma 3.15). But this topological 
space is contractible by Lemma 2.9; this fact concludes the proof just in case $T$ verifies assumption (a) of Setup 5.4.

Now, suppose that $T$ verifies hypothesis (b) of Setup 5.4 (and also that $\mathfrak{p} \subseteq \mathfrak{m}$ ). On one hand, if $\mathfrak{p} \in \mathbf{W}(J, K)$, then our coaugmented cochain complex is identically zero, whence we are done. On the other hand, if $\mathfrak{p} \notin \mathbf{W}(J, K)$, then such coaugmented cochain complex turns out to be equal to the one for computing the simplicial cohomology of the topological space $\left[q, 1_{\widehat{P}}\right.$ ) with coefficients in $Y$ (see Lemma 3.15). But this topological space is contractible by Lemma 2.9; therefore, the proof is completed.

The proof of the existence of the following spectral sequence is quite similar to the ones of Theorems 4.6 and 6.3 and it will be omitted.

Theorem 5.6. Given an inverse system $V \in \operatorname{Inv}(\widehat{P}, \mathcal{A})$, where $P$ is any finite poset, there is a 1st quadrant spectral sequence

$$
E_{2}^{i, j}=\mathbb{R}^{i} \lim _{p \in P} \mathbb{R}^{j} \mathcal{T}(V) \Longrightarrow \mathbb{R}^{i+j}\left(\lim _{p \in P} \circ \mathcal{T}\right)(V)
$$

If, in addition, there is a natural equivalence of functors

$$
\lim _{p \in P} \circ \mathcal{T} \cong T \circ \lim _{p \in P}
$$

and $V$ is acyclic with respect to the limit functor, then the previous spectral sequence can be arranged in the following manner:

$$
E_{2}^{i, j}=\mathbb{R}^{i} \lim _{p \in P} \mathbb{R}^{j} \mathcal{T}(V) \Longrightarrow \mathbb{R}^{i+j} T\left(\lim _{p \in P} V_{p}\right)
$$

Remark 5.7. It is worth mentioning here that Theorem 5.6 can be regarded as an extension of the argument pointed out in [9, Remark 8.8].

\subsection{Examples}

The goal of this part is to specialize Theorem 5.6 to several functors that fulfill the assumptions of Setup 5.4 and also satisfy the natural equivalence of functors requested in Theorem 5.6. In what follows, $J$ and $K$ will denote arbitrary ideals of $A, N$ will stand for a finitely generated $A$-module, and $V \in \operatorname{Inv}(\widehat{P}, \mathcal{A})$ being acyclic with respect to the 
limit. Moreover, we shall use the fact that filtered colimits commute with finite limits. Before going on, we have to review the following notion [6].

Definition 5.8. Let $\Phi$ be a nonempty set of ideals of $A$. It is said that $\Phi$ is a system of ideals of $A$ if, whenever $\mathfrak{a}, \mathfrak{b} \in \Phi$, there is an ideal $\mathfrak{c}$ in $\Phi$ such that $\mathfrak{c} \subseteq \mathfrak{a} \mathfrak{b}$. The reader should notice that, regarding a system of ideals $\Phi$ as a poset ordered by reverse inclusion, $\Phi$ turns out to be a filtered poset. Keeping this in mind, one can define the bifunctor $H_{\Phi}^{i}(-,-)$ by

$$
H_{\Phi}^{i}(N, M):=\operatorname{colim}_{\mathfrak{a} \in \Phi} \operatorname{Ext}_{A}^{i}(N / \mathfrak{a} N, M)
$$

- Covariant Hom: The functor $\operatorname{Hom}_{A}(N,-)$ verifies the assumptions of Setup 5.4. Indeed, it is enough to point out that

$$
\operatorname{Hom}_{A}\left(N, \lim _{p \in P} V_{p}\right) \cong \lim _{p \in P} \mathcal{H} \operatorname{om}_{A}(|N|, V)
$$

Moreover, given $\mathfrak{p} \in \operatorname{Spec}(A)$ and $\mathfrak{m} \in \operatorname{Max}(A)$ it follows, once again as a direct consequence of $[8,4.1 .7]$, that

$$
\operatorname{Hom}_{A}(N, E(A / \mathfrak{p}))_{\mathfrak{m}}=\left\{\begin{array}{l}
\operatorname{Hom}_{A_{\mathfrak{m}}}\left(N_{\mathfrak{m}}, E(A / \mathfrak{p})_{\mathfrak{m}}\right), \text { if } \mathfrak{p} \subseteq \mathfrak{m} \\
0, \text { otherwise. }
\end{array}\right.
$$

Therefore, we obtain the following spectral sequence:

$$
E_{2}^{i, j}=\mathbb{R}^{i} \lim _{p \in P} \mathcal{E x t}_{A}^{j}(|N|, V) \Longrightarrow \operatorname{Ext}_{A}^{i+j}\left(N, \lim _{p \in P} V_{p}\right)
$$

where, as usual, $|N|$ denotes the constant inverse system given by $N$ with identities on $N$ as structural morphisms.

Remark 5.9. When $I=I_{1} \cap I_{2}$ and $V=M /[*] M$ for some $A$-module $M$, this spectral sequence degenerates without assumptions to the long exact sequence

$$
\begin{aligned}
& \longrightarrow \operatorname{Ext}_{A}^{j}(N, M / I M) \longrightarrow \operatorname{Ext}_{A}^{j}\left(N, M / I_{1} M\right) \oplus \operatorname{Ext}_{A}^{j}\left(N, M / I_{2} M\right) \longrightarrow \operatorname{Ext}_{A}^{j}\left(N, M /\left(I_{1}+I_{2}\right) M\right) \\
& \longrightarrow \operatorname{Ext}_{A}^{j+1}(N, M / I M) \longrightarrow \ldots
\end{aligned}
$$


obtained after applying the functor $\operatorname{Hom}_{A}(N,-)$ to the natural short exact sequence

$$
0 \longrightarrow M / I M \longrightarrow M / I_{1} M \oplus M / I_{2} M \longrightarrow M /\left(I_{1}+I_{2}\right) M \longrightarrow 0
$$

- Generalized local cohomology: The generalized torsion functor $\Gamma_{J}(N,-)$ verifies these requirements too. It may be verified in the following way:

$$
\begin{aligned}
\Gamma_{J}\left(N, \lim _{p \in P} V_{p}\right) & \cong \operatorname{Hom}_{A}\left(N, \Gamma_{J}\left(\lim _{p \in P} V_{p}\right)\right) \cong \operatorname{Hom}_{A}\left(N, \lim _{p \in P} \mathcal{H}_{J}^{0}(V)\right) \\
& \cong \lim _{p \in P} \mathcal{H} \operatorname{om}_{A}\left(|N|, \mathcal{H}_{J}^{0}(V)\right) \cong \lim _{p \in P} \mathcal{H}_{J}^{0}(N, V)
\end{aligned}
$$

In addition, we also have to point out that, for any $\mathfrak{p} \in \operatorname{Spec}(A)$ and $\mathfrak{m} \in \operatorname{Max}(A)$,

$$
\Gamma_{J}(N, E(A / \mathfrak{p}))_{\mathfrak{m}}=\left\{\begin{array}{l}
\operatorname{Hom}_{A_{\mathfrak{m}}}\left(N_{\mathfrak{m}}, E(A / \mathfrak{p})_{\mathfrak{m}}\right), \text { if } \mathfrak{p} \in \mathrm{V}(J) \text { and } \mathfrak{p} \subseteq \mathfrak{m} \\
0, \text { otherwise. }
\end{array}\right.
$$

Remark 5.10. The ordinary torsion functor $\Gamma_{J}(-)$ also verifies the assumptions. This fact follows from the next chain of isomorphisms:

$$
\begin{aligned}
\Gamma_{J}\left(\lim _{p \in P} V_{p}\right) & \cong \operatorname{colim}_{t \in \mathbb{N}} \operatorname{Hom}_{A}\left(A / J^{t}, \lim _{p \in P} V_{p}\right) \cong \operatorname{colim}_{t \in \mathbb{N}} \lim _{p \in P} \mathcal{H} \operatorname{om}_{A}\left(\left|A / J^{t}\right|, V\right) \\
& \cong \lim _{p \in P} \operatorname{colim}_{t \in \mathbb{N}} \mathcal{H} \operatorname{om}_{A}\left(\left|A / J^{t}\right|, V\right) \cong \lim _{p \in P} \mathcal{H}_{J}^{0}(V)
\end{aligned}
$$

Furthermore, the reader should also remember, given $\mathfrak{p} \in \operatorname{Spec}(A)$ and $\mathfrak{m} \in \operatorname{Max}(A)$, that

$$
\Gamma_{J}(E(A / \mathfrak{p}))_{\mathfrak{m}}=\left\{\begin{array}{l}
E(A / \mathfrak{p})_{\mathfrak{m}}, \text { if } \mathfrak{p} \in \mathbf{V}(J) \text { and } \mathfrak{p} \subseteq \mathfrak{m} \\
0, \text { otherwise }
\end{array}\right.
$$

Therefore, we obtain the following spectral sequence:

$$
E_{2}^{i, j}=\mathbb{R}^{i} \lim _{p \in P} \mathcal{H}_{J}^{j}(|N|, V) \Longrightarrow H_{J}^{i+j}\left(N, \lim _{p \in P} V_{p}\right)
$$


Remark 5.11. When $I=I_{1} \cap I_{2}$ and $V=M /[*] M$ for some $A$-module $M$, this spectral sequence boils down to the long exact sequence

$$
\begin{aligned}
& \longrightarrow H_{J}^{j}(N, M / I M) \longrightarrow H_{J}^{j}\left(N, M / I_{1} M\right) \oplus H_{J}^{j}\left(N, M / I_{2} M\right) \longrightarrow H_{J}^{j}\left(N, M /\left(I_{1}+I_{2}\right) M\right) \\
& \longrightarrow H_{J}^{j+1}(N, M / I M) \longrightarrow \ldots
\end{aligned}
$$

obtained after applying the functor $\Gamma_{J}(N,-)$ to the natural short exact sequence

$$
0 \longrightarrow M / I M \longrightarrow M / I_{1} M \oplus M / I_{2} M \longrightarrow M /\left(I_{1}+I_{2}\right) M \longrightarrow 0
$$

- Generalized ideal transforms: The generalized Nagata's ideal transform functor $D_{J}(N,-)$ also verifies the previous assumptions. Indeed, we only have to notice that

$$
\begin{aligned}
D_{J}\left(N, \lim _{p \in P} V_{p}\right) & \cong \operatorname{colim}_{t \in \mathbb{N}} \operatorname{Hom}_{A}\left(J^{t} N, \lim _{p \in P} V_{p}\right) \\
& \cong \operatorname{colim}_{t \in \mathbb{N}} \lim _{p \in P} \mathcal{H} \operatorname{om}_{A}\left(\left|J^{t} N\right|, V\right) \cong \lim _{p \in P} \mathcal{D}_{J}(N, V) .
\end{aligned}
$$

In addition, we also have to point out that, for any $\mathfrak{p} \in \operatorname{Spec}(A)$ and $\mathfrak{m} \in \operatorname{Max}(A)$,

$$
D_{J}(N, E(A / \mathfrak{p}))_{\mathfrak{m}}=\left\{\begin{array}{l}
\operatorname{Hom}_{A_{\mathfrak{m}}}\left(N_{\mathfrak{m}}, E(A / \mathfrak{p})_{\mathfrak{m}}\right), \text { if } \mathfrak{p} \notin \mathrm{V}(J) \text { and } \mathfrak{p} \subseteq \mathfrak{m} \\
0, \text { otherwise. }
\end{array}\right.
$$

Therefore, we obtain the following spectral sequence:

$$
E_{2}^{i, j}=\mathbb{R}^{i} \lim _{p \in P} \mathbb{R}^{j} \mathcal{D}_{J}(|N|, V) \Longrightarrow \mathbb{R}^{i+j} D_{J}\left(N, \lim _{p \in P} V_{p}\right)
$$

Remark 5.12. When $I=I_{1} \cap I_{2}$ and $V=M /[*] M$ for some $A$-module $M$, the previous spectral sequence becomes the long exact sequence

$$
\begin{aligned}
& \longrightarrow \mathbb{R}^{j} D_{J}(N, M / I M) \longrightarrow \mathbb{R}^{j} D_{J}\left(N, M / I_{1} M\right) \oplus \mathbb{R}^{j} D_{J}\left(N, M / I_{2} M\right) \\
& \longrightarrow \mathbb{R}^{j} D_{J}\left(N, M /\left(I_{1}+I_{2}\right) M\right) \longrightarrow \mathbb{R}^{j+1} D_{J}(N, M / I M) \longrightarrow \ldots
\end{aligned}
$$

obtained after applying the functor $D_{J}(N,-)$ to the natural short exact sequence

$$
0 \longrightarrow M / I M \longrightarrow M / I_{1} M \oplus M / I_{2} M \longrightarrow M /\left(I_{1}+I_{2}\right) M \longrightarrow 0
$$


- Local cohomology with respect to pairs of ideals: The torsion functor $\Gamma_{J, K}$ with respect to $(J, K)$ verifies the previous requirements. Indeed, set $\widetilde{W}(J, K)$ as the set of ideals a of $A$ such that $J^{t} \subseteq \mathfrak{a}+K$ for some $t \in \mathbb{N}$. We regard $\widetilde{W}(J, K)$ as a poset with order given by reverse inclusion of ideals. In this way, applying [61, 3.2], we obtain

$$
\Gamma_{J, K}\left(\lim _{p \in P} V_{p}\right) \cong \operatorname{colim}_{\mathfrak{a} \in \widetilde{W}(J, K)} \Gamma_{\mathfrak{a}}\left(\lim _{p \in P} V_{p}\right)
$$

Combining this isomorphism with the fact that $\widetilde{W}(J, K)$ is filtered we get

$$
\begin{aligned}
\Gamma_{J, K}\left(\lim _{p \in P} V_{p}\right) & \cong \operatorname{colim}_{\mathfrak{a} \in \tilde{W}(J, K)} \Gamma_{\mathfrak{a}}\left(\lim _{p \in P} V_{p}\right) \\
& \cong \operatorname{colim}_{\mathfrak{a} \in \widetilde{W}(J, K)} \lim _{p \in P} \mathcal{H}_{\mathfrak{a}}^{0}(V) \cong \lim _{p \in P} \mathcal{H}_{J, K}^{0}(V)
\end{aligned}
$$

Moreover, we also notice that, for any $\mathfrak{p} \in \operatorname{Spec}(A)$ and $\mathfrak{m} \in \operatorname{Max}(A)$,

$$
\Gamma_{J, K}(E(A / \mathfrak{p}))_{\mathfrak{m}}=\left\{\begin{array}{l}
E(A / \mathfrak{p})_{\mathfrak{m}}, \text { if } \mathfrak{p} \in \mathbf{W}(J, K) \text { and } \mathfrak{p} \subseteq \mathfrak{m} \\
0, \text { otherwise. }
\end{array}\right.
$$

Therefore, we obtain the following spectral sequence:

$$
E_{2}^{i, j}=\mathbb{R}^{i} \lim _{p \in P} \mathcal{H}_{J, K}^{j}(V) \Longrightarrow H_{J, K}^{i+j}\left(\lim _{p \in P} V_{p}\right)
$$

Remark 5.13. When $I=I_{1} \cap I_{2}$ and $V=M /[*] M$ for some $A$-module $M$, this spectral sequence boils down to the long exact sequence

$$
0 \longrightarrow M / I M \longrightarrow M / I_{1} M \oplus M / I_{2} M \longrightarrow M /\left(I_{1}+I_{2}\right) M \longrightarrow 0
$$

obtained after applying the functor $\Gamma_{J, K}$ to the natural short exact sequence

$$
0 \longrightarrow M / I M \longrightarrow M / I_{1} M \oplus M / I_{2} M \longrightarrow M /\left(I_{1}+I_{2}\right) M \longrightarrow 0
$$

- Local cohomology with respect to inverse systems of ideals: Let $\Phi$ be a system of ideals. We claim that $\Gamma_{\Phi}(N,-)$ also verifies these requirements; indeed, it is 
enough to point out that

$$
\begin{aligned}
\Gamma_{\Phi}\left(N, \lim _{p \in P} V_{p}\right) & \cong \operatorname{colim}_{\mathfrak{a} \in \Phi} \operatorname{Hom}_{A}\left(N / \mathfrak{a} N, \lim _{p \in P} V_{p}\right) \cong \operatorname{colim}_{\mathfrak{a} \in \Phi} \lim _{p \in P} \mathcal{H o m} A(|N / \mathfrak{a} N|, V) \\
& \cong \lim _{p \in P} \operatorname{colim}_{\mathfrak{a} \in \Phi} \mathcal{H} \operatorname{Hom}_{A}(|N / \mathfrak{a} N|, V) \cong \lim _{p \in P} \mathcal{H}_{\Phi}^{0}(N, V)
\end{aligned}
$$

Furthermore, we have to point out that, for any $\mathfrak{p} \in \operatorname{Spec}(A)$ and $\mathfrak{m} \in \operatorname{Max}(A)$,

$$
\Gamma_{\Phi}(N, E(A / \mathfrak{p}))_{\mathfrak{m}}=\left\{\begin{array}{l}
\operatorname{colim}_{\mathfrak{a} \in \Phi} \operatorname{Hom}_{A_{\mathfrak{m}}}\left(N_{\mathfrak{m}} / \mathfrak{a}_{\mathfrak{m}} N_{\mathfrak{m}}, E(A / \mathfrak{p})_{\mathfrak{m}}\right), \text { if } \mathfrak{p} \subseteq \mathfrak{m} \\
0, \text { otherwise. }
\end{array}\right.
$$

Therefore, we obtain the following spectral sequence:

$$
E_{2}^{i, j}=\mathbb{R}^{i} \lim _{p \in P} \mathcal{H}_{\Phi}^{j}(|N|, V) \Longrightarrow H_{\Phi}^{i+j}\left(N, \lim _{p \in P} V_{p}\right)
$$

Remark 5.14. When $I=I_{1} \cap I_{2}$ and $V=M /[*] M$ for some $A$-module $M$, this spectral sequence boils down to the long exact sequence

$$
\begin{aligned}
& \longrightarrow H_{\Phi}^{j}(N, M / I M) \longrightarrow H_{\Phi}^{j}\left(N, M / I_{1} M\right) \oplus H_{\Phi}^{j}\left(N, M / I_{2} M\right) \longrightarrow H_{\Phi}^{j}\left(N, M /\left(I_{1}+I_{2}\right) M\right) \\
& \longrightarrow H_{\Phi}^{j+1}(N, M / I M) \longrightarrow \ldots
\end{aligned}
$$

obtained after applying the functor $\Gamma_{\Phi}(N,-)$ to the natural short exact sequence

$$
0 \longrightarrow M / I M \longrightarrow M / I_{1} M \oplus M / I_{2} M \longrightarrow M /\left(I_{1}+I_{2}\right) M \longrightarrow 0
$$

So far, in all the aforementioned examples we only picked different choices of $T$; in the following example, we also make a different choice of poset $P$.

- Local cohomology of toric face rings: Let $\Sigma \subseteq \mathbb{R}^{d}$ be a rational pointed fan with $\Sigma=\Sigma_{1} \cup \ldots \cup \Sigma_{n}$ for certain subfans $\Sigma_{j} \subseteq \Sigma$, let $\mathcal{M}_{\Sigma}$ be a monoidal complex supported on $\Sigma$, and let $P$ be the poset given by all the possible intersections of the fans $\Sigma_{j}$ ordered by inclusion. Moreover, let $\mathbb{K}$ be a field, and let $\mathbb{K}\left[\mathcal{M}_{\Sigma}\right]$ be the corresponding toric face ring, with $\mathfrak{m}$ as graded maximal ideal. In this case, if $T=\Gamma_{\mathfrak{m}}$ and $A=\mathbb{K}\left[\mathcal{M}_{\Sigma}\right]$, then for any $p \in P$ we can regard $\mathbb{K}\left[\mathcal{M}_{\Sigma_{p}}\right]$ as a $\mathbb{K}\left[\mathcal{M}_{\Sigma}\right]$-module and therefore we obtain 
the next spectral sequence:

$$
E_{2}^{i, j}=\mathbb{R}^{i} \lim _{p \in P} \mathcal{H}_{\mathfrak{m}}^{j}\left(\mathbb{K}\left[\mathcal{M}_{\Sigma_{p}}\right]\right) \Longrightarrow H_{\mathfrak{m}}^{i+j}\left(\lim _{p \in P} \mathbb{K}\left[\mathcal{M}_{\Sigma_{p}}\right]\right) .
$$

Notice that, in this specific setting, the inverse system $\left(\mathbb{K}\left[\mathcal{M}_{\Sigma_{p}}\right]\right)_{p \in P}$ is acyclic with respect to the limit functor.

Remark 5.15. When $\Sigma=\Sigma_{1} \cup \Sigma_{2}$, then the above spectral sequence boils down to the Mayer-Vietoris long exact sequence obtained in [28, 4.3].

\subsection{Enhanced structure}

This part is completely analogous with the one carried out in the homological case; indeed, our goal is to show that the spectral sequence established in Theorem 5.6 acquires a certain additional structure provided that the source inverse system has it as well. The following assumption should be compared with Assumption 4.7.

Assumption 5.16. Let $\mathcal{S} \subseteq \mathcal{A}$ be an abelian subcategory closed under subobjects, subquotients, and extensions such that, for any object $G \in \operatorname{Inv}(P, \mathcal{S}), \mathcal{T}(G) \in \operatorname{Inv}(P, \mathcal{S})$; moreover, if $M \in \operatorname{Inv}(P, \mathcal{S})$ then we suppose that there is a long exact sequence $0 \longrightarrow M \longrightarrow E^{*}$ in $\operatorname{Inv}(P, \mathcal{A})$ such that

(i) For any $j \geq 0, \mathbb{R}^{k} \mathcal{T}\left(E^{j}\right)=0$ for all $k \geq 1$.

(ii) The long exact sequence $0 \longrightarrow M \longrightarrow E^{*}$ may be regarded as an exact cochain complex in $\operatorname{Inv}(P, \mathcal{S})$.

(iii) If $A$ contains a field $\mathbb{K}$, then we also assume that, for any $N \in \mathcal{S}, \operatorname{Hom}_{A}(\mathbb{K}, N)$ is also an object of $\mathcal{S}$ such that the evaluation at 1 map $\operatorname{Hom}_{A}(\mathbb{K}, N) \longrightarrow N$ is a natural isomorphism in $\mathcal{S}$.

In this way, the main result of this part is the following:

Theorem 5.17. Suppose that $\mathcal{S} \subseteq \mathcal{A}$ satisfies part (i) and (ii) of Assumption 5.16, let $P$ be any finite poset, and let $V \in \operatorname{Inv}(\widehat{P}, \mathcal{S})$; moreover, suppose that there is a natural equivalences

$$
\lim _{p \in P} \circ \mathcal{T} \cong T \circ \lim _{p \in P}
$$


and that $V$ is acyclic with respect to the limit functor. Then, the spectral sequence obtained in Theorem 5.6

$$
E_{2}^{i, j}=\mathbb{R}^{i} \lim _{p \in P} \mathbb{R}^{j} \mathcal{T}(V) \Longrightarrow \mathbb{R}^{i+j} T\left(\lim _{p \in P} V_{p}\right)
$$

can be naturally regarded as spectral sequence in the category $\mathcal{S}$.

Now, we want to introduce the two examples we are mostly interested on.

- Graded modules: Let $A$ be a $\mathbb{Z}^{n}$-graded commutative Noetherian ring, let $T$ be either the torsion functor $\Gamma_{J}$ or the ideal transform $D_{J}$ with respect to some homogeneous ideal $J$; firstly, it is known that, given a $\mathbb{Z}^{n}$-graded $A$-module $M$, both $\Gamma_{J}(M)$ and $D_{J}(M)$ are $\mathbb{Z}^{n}$-graded $A$-modules as well [8, Chapter 13]. Secondly, Theorem 2.16 ensures that any $M \in \operatorname{Inv}\left(P,{ }^{*} \mathcal{A}\right)$ can be embedded into a long exact sequence $0 \longrightarrow M \longrightarrow E^{*}$, where $E^{k} \in \operatorname{Inv}\left(P,{ }^{*} \mathcal{A}\right)$ are made up by ${ }^{*}$ injectives objects, which are clearly acyclic with respect to the functor $T$. Finally, part (iii) also holds mainly because $\mathbb{K}$ is concentrated in degree 0.

- Modules with Frobenius action: Let $A$ be a commutative Noetherian regular domain containing an $F$-finite field $\mathbb{K}$ of prime characteristic $p$, that is, $\mathbb{K}$ is a finitedimensional $\mathbb{K}^{p}$-vector space. Let $T$ be either the torsion functor $\Gamma_{J}$ or the ideal transform $D_{J}$ with respect to any ideal $J$ of $A$, and let $A[\Theta ; F]$ be the Frobenius-skew polynomial ring. First of all, given a left $A[\Theta ; F]$-module $M$, both $\Gamma_{J}(M)$ and $D_{J}(M)$ also become in $A[\Theta ; F]$-modules with the induced action of the Frobenius on $M$ [8, Chapter 5]. Second, since $A$ is regular and contains an $F$-finite field, Kunz's Theorem ensures that $A[\Theta ; F]$ is not only a free left $A$-module but also a free right $A$-module; indeed, under our assumptions there is an isomorphism $A \Theta^{i} \cong \Theta^{i} A^{1 / p^{i}}$ (for any $i \geq 0$ ), and $A^{1 / p^{i}}$ is a flat (actually, free) $A$-module. This implies, by [44, Corollary 1.1 (2)], that any injective $A[\Theta ; F]$-module is, in particular, an injective $A$-module; notice that here we are also using that $A$ is Noetherian, which is equivalent to say that any arbitrary direct sum of injective $A$-modules is also injective.

However, notice that, as in the case of $F$-modules, part (iii) of Assumption 5.16 works when $\mathbb{K}=\mathbb{F}_{p}$ and we restrict our attention to the category of $\mathbb{F}_{p}[\Theta ; F]$-modules.

\subsection{Degeneration of cohomological spectral sequences}

Now we are in a position to provide sufficient conditions to ensure the degeneration at the $E_{2}$-sheet of the spectral sequence obtained in Theorem 5.6; namely the folllowing: 
Theorem 5.18. Let $\mathbb{K}$ be any field, let $A$ be a commutative Noetherian ring containing $\mathbb{K}$, and let $P$ be any finite poset. We further assume that the inverse system $V$ is acyclic with respect to the limit on $P$, that there is a natural equivalence of functors $\lim _{p \in P} \circ \mathcal{T} \cong T$ 。 $\lim _{p \in P}$, that for any $p \in P, \mathbb{R}^{j} T\left(V_{p}\right)=0$ up to a single value of $j$ (namely, $d_{p}$ ), and that for any $p \neq q, \operatorname{Hom}_{A}\left(\mathbb{R}^{d_{p}} T\left(V_{p}\right), \mathbb{R}^{d_{q}} T\left(V_{q}\right)\right)=0$. Then, there are canonical isomorphisms of $A$-modules

$$
\mathbb{R}^{i} \lim _{p \in P}\left(\mathbb{R}^{j} \mathcal{T}(V)\right) \cong \bigoplus_{j=d_{q}} \operatorname{Hom}_{\mathbb{K}}\left(\widetilde{H}_{i-1}\left(\left(q, 1_{\widehat{P}}\right) ; \mathbb{K}\right), \mathbb{R}^{d_{q}} T\left(V_{q}\right)\right) \cong \bigoplus_{j=d_{q}} \mathbb{R}^{d_{q}} T\left(V_{q}\right)^{\oplus M_{i, q}}
$$

where $M_{i, q}:=\operatorname{dim}_{\mathbb{K}}\left(\widetilde{H}_{i-1}\left(\left(q, 1_{\widehat{P}}\right) ; \mathbb{K}\right)\right)$; moreover, there exists a 1 st quadrant spectral sequence of the form

$$
E_{2}^{i, j}=\bigoplus_{j=d_{q}} \mathbb{R}^{d_{q}} T\left(V_{q}\right)^{\oplus M_{i, q}} \Longrightarrow \mathbb{R}^{i+j} T\left(\lim _{p \in P} V_{p}\right)
$$

which degenerates at the $E_{2}$-sheet.

Proof. Since $\mathbb{R}^{j} T\left(V_{p}\right)=0$ up to a single value of $j$ and $\operatorname{Hom}_{A}\left(\mathbb{R}^{d_{p}} T\left(V_{p}\right), \mathbb{R}^{d_{q}} T\left(V_{q}\right)\right)=$ 0 , it follows that there is a canonical isomorphism of inverse systems of $A$-modules

$$
\mathbb{R}^{j} \mathcal{T}(V) \cong \bigoplus_{j=d_{q}}\left(\mathbb{R}^{d_{q}} T\left(V_{q}\right)\right)_{q}
$$

Indeed, the inverse system $\mathbb{R}^{j} \mathcal{T}(V)$ is the one given, by the very definition of the functor $\mathcal{T}$, by $\left(\mathbb{R}^{j} T\left(V_{p}\right)\right)_{p \in P}=\left(\mathbb{R}^{d_{p}} T\left(V_{p}\right)\right)_{p \in P}\left(j=d_{p}\right)$, where the only nonzero structural morphisms are identities; these facts imply the decomposition of $\mathbb{R}^{j} \mathcal{T}(V)$ into the direct sum above.

Now, fix $i \in \mathbb{N}$. Applying the $i$ th right derived functor of the inverse limit over $P$ to the above decomposition, we get the following canonical isomorphism of $A$-modules:

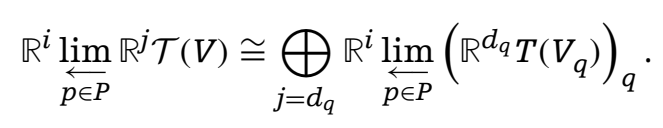


Moreover, the cohomological analog of Lemma 4.10 implies that there is a canonical isomorphism of $A$-modules:

$$
\mathbb{R}^{i}{\underset{p \in P}{(\lim }}_{\mathbb{R}^{j}} \mathcal{T}(V) \cong \bigoplus_{j=d_{q}} \widetilde{H}^{i-1}\left(\left(q, 1_{\widehat{P}}\right) ; \mathbb{R}^{d_{q}} T\left(V_{q}\right)\right)
$$

Now, since the map $\widetilde{H}^{i-1}\left(\left(q, 1_{\widehat{P}}\right) ; \mathbb{R}^{d_{q}} T\left(V_{q}\right)\right) \rightarrow \operatorname{Hom}_{\mathbb{K}}\left(\widetilde{H}_{i-1}\left(\left(q, 1_{\widehat{P}}\right) ; \mathbb{K}\right), \mathbb{R}^{d_{q}} T\left(V_{q}\right)\right)$ is a natural isomorphism of $A$-modules, one obtains the following natural isomorphism of $A$-modules:

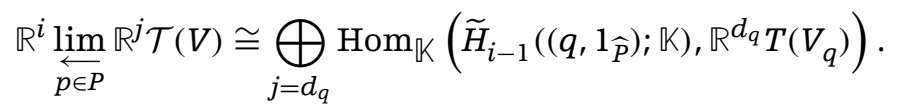

Now, set $M_{i, q}:=\operatorname{dim}_{\mathbb{K}}\left(\widetilde{H}_{i-1}\left(\left(q, 1_{\widehat{P}}\right) ; \mathbb{K}\right)\right)$, so $\widetilde{H}_{i-1}\left(\left(q, 1_{\widehat{P}}\right) ; \mathbb{K}\right) \cong \mathbb{K}^{\oplus M_{i, q}}$. As the evaluation map

$$
\operatorname{Hom}_{\mathbb{K}}\left(\mathbb{K}, \mathbb{R}^{d_{q}} T\left(V_{q}\right)\right) \longrightarrow \mathbb{R}^{d_{q}} T\left(V_{q}\right)
$$

is a canonical isomorphism of $A$-modules one obtains a natural isomorphism

$$
\operatorname{Hom}_{\mathbb{K}}\left(\widetilde{H}_{i-1}\left(\left(q, 1_{\widehat{P}}\right) ; \mathbb{K}\right), \mathbb{R}^{d_{q}} T\left(V_{q}\right)\right) \cong \mathbb{R}^{d_{q}} T\left(V_{q}\right)^{\oplus M_{i, q}}
$$

of $A$-modules and therefore we finally have a natural $A$-module isomorphism

$$
\mathbb{R}^{i} \lim _{p \in P} \mathbb{R}^{j} \mathcal{T}(V) \cong \bigoplus_{j=d_{q}} \mathbb{R}^{d_{q}} T\left(V_{q}\right)^{\oplus M_{i, q}}
$$

as claimed.

Moreover, we also want to state the cohomological analog of Corollary 4.12; in this case, the details are left to the interested reader.

Corollary 5.19. Under the assumptions of Theorem 5.18, for each $0 \leq r \leq \operatorname{cd}(T)$ there is an increasing, finite filtration $\left\{H_{k}^{r}\right\}$ of $\mathbb{R}^{r} T\left(\lim _{p \in P} V_{p}\right)$ by $A$-modules such that, for any $k \geq 0$,

$$
H_{k}^{r} / H_{k-1}^{r} \cong \bigoplus_{\left\{q \in P \mid r-k=d_{q}\right\}} \mathbb{R}^{d_{q}} T\left(V_{q}\right)^{\oplus M_{k, q}},
$$

where $M_{k, q}=\operatorname{dim}_{\mathbb{K}} \widetilde{H}^{k-d_{q-1}}\left(\left(q, 1_{\widehat{P}}\right) ; \mathbb{K}\right)$, and we follow the convention that $H_{-1}^{r}=0$.

We conclude this part writing down the enriched version (see Assumption 5.16) of Corollary 5.19; namely the following: 
Theorem 5.20. Let $\mathbb{K}$ be any field, let $A$ be a commutative Noetherian ring containing $\mathbb{K}$, let $\mathcal{S}$ be a subcategory of the category of $A$-modules satisfying Assumption 5.16, and let $P$ be any finite poset. We further assume that the inverse system $V \in \operatorname{Inv}(P, \mathcal{S})$ is acyclic with respect to the limit on $P$, that there is a natural equivalence of functors $\lim _{p \in P} \circ \mathcal{T} \cong$ $T \circ \lim _{p \in P}$, that for any $p \in P, \mathbb{R}^{j} T\left(V_{p}\right)=0$ up to a single value of $j$ (namely, $d_{p}$ ), and that for any $p \neq q, \operatorname{Hom}_{\mathcal{S}}\left(\mathbb{R}^{d_{p}} T\left(V_{p}\right), \mathbb{R}^{d_{q}} T\left(V_{q}\right)\right)=0$. Then, for each $0 \leq r \leq \operatorname{cd}(T)$ there is an increasing, finite filtration $\left\{H_{k}^{r}\right\}$ of $\mathbb{R}^{r} T\left(\lim _{p \in P} V_{p}\right)$ by objects of $\mathcal{S}$ such that, for any $k \geq 0$,

$$
H_{k}^{r} / H_{k-1}^{r} \cong \bigoplus_{\left\{q \in P \mid r-k=d_{q}\right\}} \mathbb{R}^{d_{q}} T\left(V_{q}\right)^{\oplus M_{k, q}},
$$

where we follow the convention that $H_{-1}^{r}=0$, and all these isomorphisms are isomorphisms in the category $\mathcal{S}$.

\subsubsection{A spectral sequence of local cohomology modules supported at the maximal ideal}

The aim of this part is to single out the spectral sequence produced in Theorem 5.18 in the particular case where $T=\Gamma_{\mathfrak{m}}$ and $P$ is the poset associated to a decomposition of an ideal $I \subseteq A$ (see Theorem 5.22); moreover, we also provide specific examples where the assumptions required in the statement of this result are fulfilled. We also want to point out that, later in this paper (see Sections 7 and 8), we focus on this spectral sequence in order to, on the one hand, obtain some Hochster-type decompositions of local cohomology modules and, on the other hand, study the extension problems attached to the filtration produced by its degeneration.

We start with the following auxiliary result.

Lemma 5.21. Let $A$ be any commutative Noetherian ring, and let $I_{p}, I_{q}$ be two ideals of $A$ contained in a fixed maximal one (say, $\mathfrak{m}$ ) such that $I_{q} \nsubseteq \mathfrak{p}$ for any prime ideal $\mathfrak{p} \supseteq I_{p}$ such that $\operatorname{dim}(A / \mathfrak{p})=d_{p}$. Then, $\operatorname{Hom}_{A}\left(H_{\mathfrak{m}}^{d_{p}}\left(A / I_{p}\right), H_{\mathfrak{m}}^{d_{q}}\left(A / I_{q}\right)\right)=0$.

Proof. First of all, in order to simplify notation, set $H_{p}:=H_{\mathfrak{m}}^{d_{p}}\left(A / I_{p}\right)$ and $H_{q}:=$ $H_{\mathfrak{m}}^{d_{q}}\left(A / I_{q}\right)$; we assume, to get a contradiction, that there is a $0 \neq \psi \in \operatorname{Hom}_{A}\left(H_{p}, H_{q}\right)$. Write $\operatorname{Att}_{A}\left(H_{p}\right)=\left\{\mathfrak{p}_{1}, \ldots, \mathfrak{p}_{s}\right\}$, where Att denotes the set of attached primes as defined, for instance, in [8, 7.2]; in this way, we get the following commutative square:

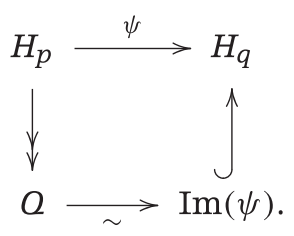


Here, $Q:=H_{p} / \operatorname{ker}(\psi)$ and the bottom isomorphism is the one provided by the First Isomorphism Theorem. Moreover, $\operatorname{Att}_{A}(Q) \subseteq \operatorname{Att}_{A}\left(H_{p}\right)$ (indeed, this fact follows from $[8,7.2 .6])$ and $\operatorname{Att}_{A}\left(H_{p}\right)=\left\{\mathfrak{p}_{1}, \ldots, \mathfrak{p}_{s}\right\}$; since $Q \neq 0$ because we are assuming that $\psi \neq 0$, we can assume, without loss of generality, that there is $1 \leq r \leq s$ such that $\operatorname{Att}_{A}(Q)=$ $\left\{\mathfrak{p}_{1}, \ldots, \mathfrak{p}_{r}\right\}$. Moreover, as $Q \cong \operatorname{Im}(\psi)$, it implies that $\operatorname{Att}_{A}(\operatorname{Im}(\psi))=\left\{\mathfrak{p}_{1}, \ldots, \mathfrak{p}_{r}\right\}$. On the other hand, since $\operatorname{Im}(\psi) \subseteq H_{q}$, it is clear that

$$
\sqrt{\left(0:_{A} H_{q}\right)} \subseteq \sqrt{\left(0:_{A} \operatorname{Im}(\psi)\right)}
$$

regardless, combining $[8,7.2 .11]$ and the foregoing facts it follows that

$$
\sqrt{I_{q}}=\sqrt{\left(0:_{A} H_{q}\right)} \subseteq \sqrt{\left(0:_{A} \operatorname{Im}(\psi)\right)}=\mathfrak{p}_{1} \cap \ldots \cap \mathfrak{p}_{r}
$$

But this contradicts our assumption that $I_{q} \nsubseteq \mathfrak{p}$ for any prime ideal $\mathfrak{p} \supseteq I_{p}$ such that $\operatorname{dim}(A / \mathfrak{p})=d_{p}$, whence $\psi$ must be zero; the proof is therefore completed.

Now, we are ready to establish the main result of this part; the reader will easily note that, in the statement, we do not need to require any vanishing of Hom's because of Lemma 5.21 .

Theorem 5.22. Let $\mathbb{K}$ be any field, let $A$ be any commutative Noetherian ring containing $\mathbb{K}$, and let $P$ be the poset given in Example 2.1; suppose that all of them are contained in a certain maximal ideal of $A$ (namely, $m$ ). We further assume that, for any $p \in P, A / I_{p}$ is a Cohen-Macaulay ring and that, for any $p \neq q, I_{q}$ is not contained in any minimal prime of $I_{p}$ (this holds, for instance, if one supposes that all the $A / I_{p} s$ are Cohen-Macaulay domains), and that the inverse system $A /[*]$ is acyclic with respect to the limit. Then, there exists a 1st quadrant spectral sequence of the form

$$
E_{2}^{i, j}=\bigoplus_{j=d_{q}} H_{\mathfrak{m}}^{d_{q}}\left(A / I_{q}\right)^{\oplus M_{i, q}} \Longrightarrow H_{\mathfrak{m}}^{i+j}\left(\lim _{p \in P} A / I_{p}\right)
$$

where $M_{i, q}=\operatorname{dim}_{\mathbb{K}} \widetilde{H}^{i-d_{q}-1}\left(\left(q, 1_{\widehat{P}}\right) ; \mathbb{K}\right)$. Moreover, this spectral sequence degenerates at the $E_{2}$-sheet and for each $0 \leq r \leq \operatorname{dim}(A)$ there is an increasing, finite filtration $\left\{H_{k}^{r}\right\}$ of $H_{\mathfrak{m}}^{r}\left(\lim _{p \in P} A / I_{p}\right)$ by $A$-modules such that, for any $k \geq 0$,

$$
H_{k}^{r} / H_{k-1}^{r} \cong \bigoplus_{\left\{q \in P \mid r-k=d_{q}\right\}} H_{\mathfrak{m}}^{d_{q}}\left(A / I_{q}\right)^{\oplus M_{k, q}}
$$


where we follow the convention that $H_{-1}^{r}=0$.

Some specific situations where the assumptions of Theorem 5.22 are fulfilled are the following:

- Squarefree monomial ideals: When $I$ is a squarefree monomial ideal it admits a minimal primary decomposition in terms of prime ideals generated by variables, which is a family of prime ideals closed under sum. Therefore, under these assumptions $P$ is made up by prime ideals generated by linear forms, which also verify all the assumptions of Theorem 5.22.

- Central arrangements of linear varieties: More generally, let $I$ be the defining ideal of a central arrangement of linear varieties over any field $\mathbb{K}$; in this case, we also have that $I$ admits a minimal primary decomposition in terms of prime ideals generated by linear forms, which is a family of prime ideals closed under sum. Therefore, under these assumptions $P$ is made up by prime ideals generated by linear forms, which also verify all the assumptions of Theorem 5.22 up to the fact that, in general, we cannot guarantee that $A /[*]$ is acyclic with respect to the limit functor.

- Monomial ideals in regular sequences: Let $A$ be a commutative Noetherian ring containing a field $\mathbb{K}$, and let $y_{1}, \ldots, y_{n}$ be an $A$-regular sequence contained in the Jacobson radical of $A$. Moreover, let $I:=J \mathbb{K}\left[Y_{1}, \ldots, Y_{n}\right]$, where $\mathbb{K}\left[Y_{1}, \ldots, Y_{n}\right] \stackrel{\psi}{\longrightarrow} A$ is the map of $\mathbb{K}$-algebras that sends each indeterminate $Y_{i}$ to $Y_{i}$, and $J$ is a monomial ideal in the usual sense. Thus, since $\psi$ is flat $[57,2.1]$, it follows that Theorem 5.22 can also be applied in this setting; indeed, it is known that $I$ admits a unique primary decomposition in terms of monomial (not necessarily prime) ideals in $A$, and that this decomposition is determined by the corresponding decomposition of $J$ inside $\mathbb{K}\left[Y_{1}, \ldots, Y_{n}\right][24$, Theorem 4.9 and Corollary 4.12]. Moreover, flatness of $\psi$ implies, because local cohomology commutes with flat base change, that if one denotes by $I_{p}$ one of the sums of the primary components of $I$, then one has that $A / I_{p}$ is Cohen-Macaulay. Finally, the condition that, for any $p \neq q, I_{q}$ is not contained in any minimal prime of $I_{p}$ is immediate from the fact that the decomposition of $I$ is determined by the corresponding decomposition of $J$ inside $\mathbb{K}\left[Y_{1}, \ldots, Y_{n}\right]$.

- Monomial ideals in semigroup rings: Let $Q$ be an affine semigroup [41, 7.4], let $\mathbb{K}$ be any field, and suppose that the semigroup ring $A=\mathbb{K}[Q]$ is either normal or simplicial and Cohen-Macaulay. Moreover, let $I \subseteq \mathbb{K}[Q]$ be a squarefree monomial ideal $[41,7.9]$. We claim that Theorem 5.22 can also be applied in this case; indeed, $I$ admits a minimal primary decomposition in terms of monomial prime ideals [41, 7.13]. 
In addition, for any monomial prime ideal $\mathfrak{p}$ it is known (see [11, 6.3.5], [65, Paragraph below Remark 3.4] in the normal case, and $[66,2.4]$ in the remainder case) that $A / \mathfrak{p}$ is a Cohen-Macaulay ring. Therefore, the poset $P$ is made up by Cohen-Macaulay monomial prime ideals, which is just what we need to check.

- Toric face rings: Let $\Sigma \subseteq \mathbb{R}^{d}$ be a rational pointed fan, let $\mathcal{M}_{\Sigma}$ be a CohenMacaulay monoidal complex supported on $\Sigma$, let $\mathbb{K}$ be a field, and let $A=\mathbb{K}\left[\mathcal{M}_{\Sigma}\right]$ be the corresponding toric face ring (see [28, p. 251] for unexplained terminology). We claim that Theorem 5.22 can also be applied in this case; indeed, since the sum of $\mathbb{Z}^{d}$-graded prime ideals of $A$ is again a $\mathbb{Z}^{d}$-graded prime ideal [28, Lemma 2.3 (i)] and that there is a bijection between the set of nonempty cones of $\Sigma$ and the set of $\mathbb{Z}^{d}$-graded prime ideals of $A\left[28\right.$, Lemma 2.1] it follows, since every $\mathbb{K}\left[M_{C}\right]$ is Cohen-Macaulay for any cone $C \in \Sigma$, that the poset $P$, made up by all the possible $\mathbb{Z}^{d}$-graded prime ideals of $A$, is closed under sum and satisties that, for any $p \in P, A / I_{p} \cong \mathbb{K}\left[M_{C}\right]$ is a Cohen-Macaulay domain, which is just what we need to check.

\section{Homological Spectral Sequences Associated to Inverse Systems}

The formalism given in Section 4 can be used to provide several examples of homological spectral sequences of local cohomology modules. However, it cannot be applied in the case that $T_{[*]}=\operatorname{Hom}_{A}(A /[*],-)$ so we cannot construct a spectral sequence of the form

$$
E_{2}^{-i, j}=\mathbb{L}_{i} \operatorname{colim}_{p \in P} \operatorname{Ext}_{A}^{j}\left(A / I_{p}, M\right) \Longrightarrow \operatorname{Ext}_{A}^{j-i}(A / I, M)
$$

as it was wrongly stated in [4, 1.4(iii)]. In this section, following a similar approach to the one given in Section 5, we will provide the right formalism to obtain a spectral sequence for Ext modules.

First of all we will show that the key Lemma 4.4 is no longer true for the functor $T_{[*]}=\operatorname{Hom}_{A}(A /[*],-)$ just because it does not satisfy the conditions of Setup 4.3.

Counterargument 1 . Let $\mathbb{K}$ be any field, set $A:=\mathbb{K} \llbracket x, y, z \rrbracket$ and

$$
I:=\langle x y, x z, y z\rangle=\langle x, y\rangle \cap\langle x, z\rangle \cap\langle y, z\rangle=I_{1} \cap I_{2} \cap I_{3} .
$$

Moreover, set $E:=E_{A}(\mathbb{K})$ as a choice of injective hull of $\mathbb{K}$ over $A$; our goal in this example is to compute explicitly the following augmented chain complex:

$$
\operatorname{Roos}_{*}\left(\operatorname{Hom}_{A}(A /[*], E)\right) \longrightarrow \operatorname{Hom}_{A}(A / I, E) \longrightarrow 0 \text {. }
$$


In addition, since it is noteworthy that, for any ideal $J$ of $A$, there is a canonical isomorphism of $A$-modules $\operatorname{Hom}_{A}(A / J, E) \cong\left(0:_{E} J\right)$; it turns out that (6) is canonically isomorphic to the next augmented chain complex $\operatorname{Roos}_{*}\left(\left(0:_{E}[*]\right)\right) \longrightarrow\left(0:_{E} I\right) \longrightarrow 0$, which, in this case, is nothing but

$$
0 \longrightarrow \operatorname{Roos}_{1}\left(\left(0::_{E}[*]\right)\right) \stackrel{d_{1}}{\longrightarrow} \operatorname{Roos}_{0}((0: E[*])) \stackrel{d_{0}}{\longrightarrow}\left(0::_{E} I\right) \longrightarrow 0 .
$$

So, our aim is to calculate explicitly (7). Firstly, we determine its spots: on the one hand, its 0th spot is $\operatorname{Roos}_{0}\left(\left(0:_{E}[*]\right)\right)=\left(0:_{E} \mathfrak{m}\right) \oplus\left(0:_{E} I_{3}\right) \oplus\left(0:_{E} I_{2}\right) \oplus\left(0:_{E} I_{1}\right)$. On the other hand, its 1 th spot is

$$
\operatorname{Roos}_{1}\left(\left(0:_{E}[*]\right)\right)=\left(0:_{E} \mathfrak{m}\right) \oplus\left(0:_{E} \mathfrak{m}\right) \oplus\left(0:_{E} \mathfrak{m}\right)
$$

Now, we have to compute its differentials; namely, $d_{0}$ and $d_{1}$.

(i) The 0th differential turns out to be

$$
\begin{aligned}
& \left(0::_{E} \mathfrak{m}\right) \oplus\left(0:_{E} I_{3}\right) \oplus\left(0:_{E} I_{2}\right) \oplus\left(0:_{E} I_{1}\right) \stackrel{d_{0}}{\longrightarrow}\left(0:_{E} I\right) \\
& \left(a, a_{1}, a_{2}, a_{3}\right) \longmapsto-a+a_{3}-a_{2}+a_{1} .
\end{aligned}
$$

(ii) The 1st differential $d_{1}$ is given by

$$
\begin{aligned}
& \left(0:_{E} \mathfrak{m}\right) \oplus\left(0:_{E} \mathfrak{m}\right) \oplus\left(0:_{E} \mathfrak{m}\right) \stackrel{d_{1}}{\longrightarrow}\left(0:_{E} \mathfrak{m}\right) \oplus\left(0:_{E} I_{3}\right) \oplus\left(0:_{E} I_{2}\right) \oplus\left(0:_{E} I_{1}\right) \\
& \left(b_{3}, b_{2}, b_{1}\right) \longmapsto\left(0, b_{2}-b_{3}, b_{1}-b_{3}, b_{1}-b_{2}\right) .
\end{aligned}
$$

Summing up, the augmented chain complex (7) is the one induced by the augmented chain complex

$$
0 \longrightarrow E^{\oplus 3} \stackrel{A_{1}}{\longrightarrow} E^{\oplus 4} \stackrel{A_{0}}{\longrightarrow} E \longrightarrow 0
$$

where $A_{0}:=\left(\begin{array}{llll}-1 & 1 & -1 & 1\end{array}\right)$ and

$$
A_{1}:=\left(\begin{array}{ccc}
0 & 0 & 0 \\
-1 & 1 & 0 \\
-1 & 0 & 1 \\
0 & -1 & 1
\end{array}\right)
$$

Applying Matlis duality $(-)^{\vee}$, one obtains the following coaugmented cochain complex:

$0 \longrightarrow A / I \longrightarrow A / \mathfrak{m} \oplus A / I_{3} \oplus A / I_{2} \oplus A / I_{1} \longrightarrow A / \mathfrak{m} \oplus A / \mathfrak{m} \oplus A / \mathfrak{m} \longrightarrow 0$ 
As the reader can easily note, this coaugmented cochain complex is the induced one given by the next complex $0 \longrightarrow A \stackrel{A_{0}^{t}}{\longrightarrow} A^{\oplus 4} \stackrel{A_{1}^{t}}{\longrightarrow} A^{\oplus 3} \longrightarrow 0$. Regardless, neither the previous lifted complex nor the induced one is exact. Indeed, we have checked that the lifted complex is not exact using Macaulay2 [23]. Of course, the reader might think that perhaps the lifted complex is not exact, but the induced complex after taking equivalence classes is so. Unfortunately, this is not the case, because $(\operatorname{cls}(1), \operatorname{cls}(x), \operatorname{cls}(y), \operatorname{cls}(z))$ is a member of the kernel of the map given by $A_{1}^{t}$ that does not belong to the image of the map given by $A_{0}^{t}$.

\subsection{Construction of homological spectral sequences}

The setup we need for the appropriate spectral sequence is the following:

Setup 6.1. Let $\mathcal{A} \stackrel{T}{\longrightarrow} \mathcal{A}$ be a contravariant, left exact functor, and let $P$ be any finite poset. Carrying over $T$, we produce a new functor (namely, $\mathcal{T}$ ) in the following manner:

$$
\begin{aligned}
& \operatorname{Inv}(P, \mathcal{A}) \stackrel{\mathcal{T}}{\longrightarrow} \operatorname{Dir}(P, \mathcal{A}) \\
& G=\left(G_{p}\right)_{p \in P} \longmapsto\left(T\left(G_{p}\right)\right)_{p \in P} .
\end{aligned}
$$

Moreover, we also assume that $T$ commutes with finite direct sums and that $T(A)=Z$ for some $A$-module $Z$; in particular, one has that $\operatorname{colim}_{p \in P} \mathcal{T}\left(A_{\leq q}\right)=Z$, where $q \in P$ (notice that $Z$ only depends on $T$ but not on $q$ ).

The following lemma will provide the abutment of the spectral sequence we want to construct.

Lemma 6.2. Let $G$ be a projective object of $\operatorname{Inv}(P, \mathcal{A})$ of the form

$$
G=\bigoplus_{j \in J} A_{\leq q_{j}}
$$

where $J$ is a finite index set and $q_{j} \in P$ (see Theorem 2.17). Then, the augmented chain complex

$$
\operatorname{Roos}_{*}(\mathcal{T}(G)) \longrightarrow\left(\operatorname{colim}_{p \in P} \circ \mathcal{T}\right)(G) \longrightarrow 0
$$

is exact. 
Proof. Since $\operatorname{Roos}_{*}, \mathcal{T}$ and the colimit functor commutes with finite direct sums we may suppose, without loss of generality, that $G=A_{\leq q}$ for some fixed $q \in P$; regardless, in this case, our augmented chain complex is exactly the one for computing the simplicial homology of the interval $\left[q, 1_{\widehat{P}}\right.$ ) (indeed, notice that we have to take this interval because $\mathcal{T}$ is contravariant) with coefficients in $Z$. But $[q, 1 \widehat{P})$ is contractible by Lemma 2.9 ; the proof is therefore completed.

The following result provides the announced spectral sequence; since its proof is almost verbatim to the one given in Theorem 4.6, it will be skipped.

Theorem 6.3. Given an inverse system $V \in \operatorname{Inv}(\widehat{P}, \mathcal{A})$ that is acyclic with respect to the limit functor, there is a 1 st quadrant spectral sequence

$$
E_{2}^{-i, j}=\mathbb{L}_{i} \operatorname{colim}_{p \in P} \mathbb{R}^{j} \mathcal{T}(V) \Longrightarrow H^{j-i}\left(\operatorname{colim}_{p \in P} \circ \mathcal{T}\right)(V)
$$

where the abutment denotes the cohomology of the cochain complex

$$
0 \longrightarrow \operatorname{colim}_{p \in P} \mathcal{T}(V) \longrightarrow \operatorname{colim}_{p \in P} \mathcal{T}\left(F_{0}\right) \longrightarrow \operatorname{colim}_{p \in P} \mathcal{T}\left(F_{1}\right) \longrightarrow \ldots
$$

and $\ldots \longrightarrow F_{1} \longrightarrow F_{0} \longrightarrow V \longrightarrow 0$ denotes a projective resolution of $V$ in $\operatorname{Inv}(P, \mathcal{A})$, where any $F_{i}$ is made up by direct summands of the form $A_{\leq p}(p \in P)$.

\subsection{Examples}

Of course, the example we are mainly interested on is the following one:

- Contravariant Hom: Let $N$ be an arbitrary $A$-module. Then, the functor $\operatorname{Hom}_{A}(-, N)$ is clearly left exact, contravariant, and commutes with finite direct sums; whence $\operatorname{Hom}_{A}(-, N)$ can be regarded as a particular case of Setup 6.1. Furthermore, given an inverse system $V \in \operatorname{Inv}(\widehat{P}, \mathcal{A})$ that is acyclic with respect to the limit functor we have a canonical isomorphism

$$
\operatorname{Hom}_{A}\left(\lim _{p \in P} V_{p}, N\right) \cong \operatorname{colim}_{p \in P} \mathcal{H} \operatorname{om}_{A}(V,|N|)
$$


where $|N|$ is the constant inverse system given by $N$ with identities as structural maps. Therefore, we obtain the spectral sequence:

$$
E_{2}^{-i, j}=\mathbb{L}_{i} \operatorname{colim}_{p \in P} \mathcal{E x t}_{A}^{j}(V,|N|) \Longrightarrow \operatorname{Ext}_{A}^{j-i}\left(\lim _{p \in P} V_{p}, N\right)
$$

Remark 6.4. When $I=I_{1} \cap I_{2}$ and $V=M /[*] M$ for some $A$-module $M$, this spectral sequence boils down to the long exact sequence

$$
\begin{aligned}
& \longrightarrow \operatorname{Ext}_{A}^{i}\left(M /\left(I_{1}+I_{2}\right) M, N\right) \longrightarrow \operatorname{Ext}_{A}^{i}\left(M / I_{1} M, N\right) \oplus \operatorname{Ext}_{A}^{i}\left(M / I_{2} M, N\right) \longrightarrow \operatorname{Ext}_{A}^{i}(M / I M, N) \\
& \longrightarrow \operatorname{Ext}_{A}^{i+1}\left(M /\left(I_{1}+I_{2}\right) M, N\right) \longrightarrow \ldots
\end{aligned}
$$

obtained after applying the functor $\operatorname{Hom}_{A}(-, N)$ to the natural short exact sequence:

$$
0 \longrightarrow M / I M \longrightarrow M / I_{1} M \oplus M / I_{2} M \longrightarrow M /\left(I_{1}+I_{2}\right) M \longrightarrow 0
$$

\subsection{Degeneration of homological spectral sequences}

Carrying out the same strategy used to produce Theorem 5.18 and Corollary 5.19, we obtain the following pair of results, which involve the spectral sequence constructed in Theorem 6.3; the details are left to the interested reader.

Theorem 6.5. Let $\mathbb{K}$ be any field, let $A$ be a commutative Noetherian ring containing $\mathbb{K}$, let $P$ be any finite poset, and let $T$ and $\mathcal{T}$ be as in Setup 6.1. We further assume that the inverse system $V$ is acyclic with respect to the limit on $P$, that there is a natural equivalence of functors

$$
\operatorname{colim}_{p \in P} \circ \mathcal{T} \cong T \circ \lim _{p \in P}
$$

that for any $p \in P, \mathbb{R}^{j} T\left(V_{p}\right)=0$ up to a single value of $j$ (namely, $h_{p}$ ), and that for any $p \neq q, \operatorname{Hom}_{A}\left(\mathbb{R}^{h_{p}} T\left(V_{p}\right), \mathbb{R}^{h_{q}} T\left(V_{q}\right)\right)=0$. Then, there exists a 3rd quadrant spectral sequence of the form:

$$
E_{2}^{-i, j}=\bigoplus_{j=h_{q}} \mathbb{R}^{h_{q}} T\left(V_{q}\right)^{\oplus m_{i, q}} \Longrightarrow \mathbb{R}^{j-i} T\left(\lim _{p \in P} V_{p}, N\right)
$$

where $m_{i, q}:=\operatorname{dim}_{\mathbb{K}}\left(\widetilde{H}_{i-h_{q-1}}\left(\left(q, 1_{\widehat{P}}\right) ; \mathbb{K}\right)\right)$. Moreover, this spectral sequence degenerates at the $E_{2}$-sheet. 
Corollary 6.6. Under the assumptions of Theorem 6.5, for each $0 \leq r \leq \operatorname{cd}(T)$ there is an increasing, finite filtration $\left\{H_{k}^{r}\right\}$ of $\mathbb{R}^{r} T\left(\lim _{p \in P} V_{p}\right)$ by $A$-modules such that, for any $k \geq 0$,

$$
H_{k}^{r} / H_{k-1}^{r} \cong \bigoplus_{\left\{q \in P \mid r-k=h_{q}\right\}} \mathbb{R}^{h_{q}} T\left(V_{q}\right)^{\oplus m_{k, q}}
$$

where we follow the convention that $H_{-1}^{r}=0$.

We want to single out here that we plan to use Corollary 6.6 to provide a certain decomposition of the so-called deficiency modules (see Example 7.4).

\section{Some Hochster-Type Decompositions}

A celebrated result of Hochster provides a decomposition of the local cohomology modules $H_{\mathfrak{m}}^{r}(A / I)$ in terms of $H_{\mathfrak{m}}^{r}\left(A / I_{p}\right)$ in the case that $I$ is a squarefree monomial ideal in the polynomial ring $A=\mathbb{K}\left[x_{1}, \cdots, x_{d}\right]$ over a field $\mathbb{K}$. This formula was generalized, on the one hand, to arbitrary monomial ideals by Takayama in [62, Theorem 1] (see also [9, Corollary 2.3]) and, on the other hand, to toric face rings by Brun et al. in [10].

Moreover, Mustaţă [42, Theorem 2.1 and Corollary 2.2] and Terai [63] (see also [41, Corollary 13.16]) provide a decomposition of the local cohomology modules $H_{I}^{r}(A)$ in terms of $H_{I_{p}}^{r}(A)$ in the case that $I$ is a squarefree monomial ideal in the polynomial ring $A=\mathbb{K}\left[x_{1}, \cdots, x_{d}\right]$ over a field $\mathbb{K}$.

The goal of this section is, on the one hand, to establish a decomposition of local cohomology modules such that, in the case that $I=I_{\Delta}$ is a Stanley-Reisner ideal, is just the classical Hochster's decomposition of the local cohomology of a StanleyReisner ring [41,13.13]; on the other hand, we also produce a decomposition of local cohomology modules such that, in the case that $I=I_{\Delta}$ is a Stanley-Reisner ideal is just Mustaţă-Terai's formula. In particular, we obtain a decomposition of $H_{I_{\Sigma}}^{r}(A)$, where $I_{\Sigma}$ is the defining ideal of certain toric face rings in the polynomial ring $A=\mathbb{K}\left[x_{1}, \cdots, x_{d}\right]$ over a field $\mathbb{K}$ of prime characteristic (see the Examples after Theorem 4.17 to realize why we have to restrict ourselves to this situation); to the best of our knowledge, this is the 1st time this formula appears in the literature.

First of all, we want to start with our Hochster-type decompositions; indeed, the 1st main result of this section is the following:

Consider the cohomological spectral sequences constructed in Section 5. In Theorem 5.18 we produced sufficient conditions for the spectral sequence to degenerate 
at the $E_{2}$-page. In this case we obtained the following:

$$
E_{2}^{i, j}=\bigoplus_{j=d_{q}} \mathbb{R}^{d_{q}} T\left(V_{q}\right)^{\oplus M_{i, q}} \Longrightarrow \mathbb{R}^{i+j} T\left(\lim _{p \in P} V_{p}, N\right),
$$

where $M_{i, q}=\operatorname{dim}_{\mathbb{K}} \widetilde{H}^{i-d_{q-1}}\left(\left(q, 1_{\widehat{P}}\right) ; \mathbb{K}\right)$. Moreover, according to Corollary 5.19 we have a collection of short exact sequences (for some $b \in \mathbb{N}$ )

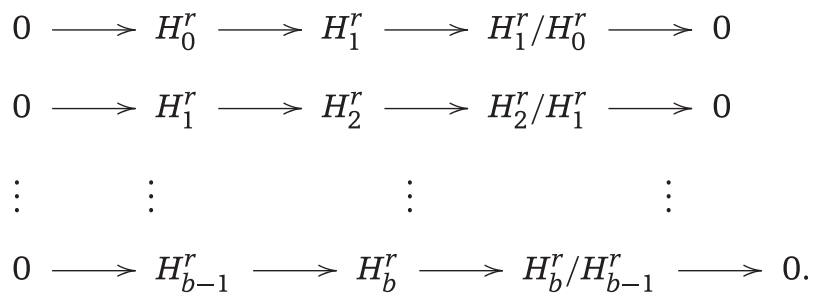

such that, for each $r$ the quotients $H_{k}^{r} / H_{k-1}^{r}$ can be decomposed in the following manner:

$$
H_{k}^{r} / H_{k-1}^{r} \cong \bigoplus_{\left\{q \in P \mid r-k=d_{q}\right\}}\left(\mathbb{R}^{d_{q}} T\left(V_{q}\right) \otimes_{\mathbb{K}} \widetilde{H}_{k-1}\left(\left(q, 1_{\widehat{P}}\right) ; \mathbb{K}\right)\right)
$$

These short exact sequences split as $\mathbb{K}$-vector spaces so we obtain the following result.

Theorem 7.1. Let $\mathbb{K}$ be a field, let $A$ be a commutative Noetherian ring containing $\mathbb{K}$, let $T$ and $\mathcal{T}$ be as in Setup 5.4, and let $P$ be any finite poset. Moreover, assume that $V \in \operatorname{Inv}(\widehat{P}, \mathcal{A})$ is acyclic with respect to the limit, that there is a natural equivalence of functors $\lim _{p \in P} \circ \mathcal{T} \cong T \circ \lim _{p \in P}$, that for any $p \in P, \mathbb{R}^{j} T\left(V_{p}\right)=0$ up to a single value of $j$ (namely, $d_{p}$ ), and that, for any $p \neq q, \operatorname{Hom}_{A}\left(\mathbb{R}^{d_{p}} T\left(V_{p}\right), \mathbb{R}^{d_{q}} T\left(V_{q}\right)\right)=0$. Then, there is a K-vector space isomorphism

$$
\mathbb{R}^{j} T\left(\lim _{p \in P} V_{p}\right) \cong \bigoplus_{q \in P} \mathbb{R}^{d_{q}} T\left(V_{q}\right)^{\oplus M_{j, q}},
$$

where $M_{j, q}=\operatorname{dim}_{\mathbb{K}} \widetilde{H}^{j-d_{q-1}}\left(\left(q, 1_{\widehat{P}}\right) ; \mathbb{K}\right)$.

Hochster's formula boils down to the case where $A=\mathbb{K}\left[x_{1}, \cdots, x_{n}\right]$ is a polynomial ring, $T=\Gamma_{\mathfrak{m}}, \mathfrak{m}$ is the graded maximal ideal of $A$, and $V=A /[*]$ is the inverse system associated to a Stanley-Reisner ring $A / I$. Of course, the result still holds true for central arrangements of linear varieties satisfying that $A /[*]$ is acyclic with respect to the limit. Brun et al. gave a generalization of Hochster's formula in [10, 4.1]. 
Their result is the specialization of Theorem 7.1 to the case where $T=\Gamma_{J}$ for some ideal $J \subseteq A$. Namely, the decomposition can be written down in the following way:

$$
H_{J}^{j}\left(\lim _{p \in P} V_{p}\right) \cong \bigoplus_{q \in P} H_{J}^{d_{q}}\left(V_{q}\right)^{\oplus M_{j, q}}
$$

The same game can be played for the homological spectral sequences constructed in Section 6. In Theorem 6.5 we produced sufficient conditions for their degeneration so we may obtain the following decomposition result; the details of the proof are left to the interested reader.

Theorem 7.2. Under the assumptions of Theorem 6.5, there is a $\mathbb{K}$-vector space isomorphism

$$
\mathbb{R}^{j} T\left(\lim _{p \in P} V_{p}\right) \cong \bigoplus_{q \in P} \mathbb{R}^{h_{q}} T\left(V_{q}\right)^{\oplus m_{j, q}}
$$

where $m_{j, q}=\operatorname{dim}_{\mathbb{K}} \widetilde{H}_{j-h_{q}-1}\left(\left(q, 1_{\widehat{P}}\right) ; \mathbb{K}\right)$.

Before applying this result to obtain a certain decomposition of deficiency modules, we want to state the following technical result, which is a direct consequence of Lemma 5.21 using local duality.

Lemma 7.3. Let $(A, \mathfrak{m})$ be a commutative Noetherian local ring where there exists a canonical module $\omega_{A}$, (so, $A$ is a Cohen-Macaulay ring that can be expressed as homomorphic image of a Gorenstein local $\operatorname{ring} B$ of dimension $d$ ) let $I_{p}$ and $I_{q}$ be two ideals of $A$ of finite projective dimension such that $I_{q} \nsubseteq \mathfrak{p}$ for any prime ideal $\mathfrak{p} \supseteq I_{p}$ such that $\operatorname{dim}(A / \mathfrak{p})=d_{p}$. Then, $\operatorname{Hom}_{A}\left(\operatorname{Ext}_{A}^{d-d_{q}}\left(A / I_{q}, \omega_{A}\right), \operatorname{Ext}_{A}^{d-d_{p}}\left(A / I_{p}, \omega_{A}\right)\right)=0$.

Now, we want to single out the following interesting particular case:

Example 7.4. Suppose that $A$ is a commutative Noetherian local ring containing a field $\mathbb{K}$ where there exists a canonical module $\omega_{A}$ (remember that this assumption implies that $A$ is a Cohen-Macaulay ring that can be expressed as homomorphic image of a local Gorenstein ring $B$ of dimension $d$ ); given any finitely generated $A$-module $N$, its $j$ th deficiency module can be defined as

$$
K^{j}(N):=\operatorname{Ext}_{A}^{d-j}\left(N, \omega_{A}\right) .
$$


Now, assume that $P$ is the poset given by a decomposition of an ideal $I \subseteq A$ as usual such that $A /[*]$ is acyclic with respect to the limit and that $\lim _{p \in P} A / I_{p}$ is a finitely generated $A$-module, such that, for any $p \in P, A / I_{p}$ is a Cohen-Macaulay ring with finite projective dimension, and such that, for any $p \neq q, I_{q} \nsubseteq \mathfrak{p}$ for any prime ideal $\mathfrak{p} \supseteq I_{p}$ such that $\operatorname{dim}(A / \mathfrak{p})=d_{p}$. Then, the decomposition obtained in Theorem 7.2 boils down to the following isomorphism of $\mathbb{K}$-vector spaces:

$$
K^{j}\left(\lim _{p \in P} A / I_{p}\right) \cong \bigoplus_{q \in P} K^{\operatorname{dim}\left(A / I_{q}\right)}\left(A / I_{q}\right)^{\oplus m_{j, q}}
$$

Indeed, this is a consequence of Lemma 7.3 jointly with the fact that, since, for any $p \in P, A / I_{p}$ is Cohen-Macaulay, one has that $K^{j}\left(A / I_{p}\right)=0$ up to a single value of $j$.

Finally, we can also obtain a similar result for our starting homological spectral sequences; namely the following:

Theorem 7.5. Let $A$ be a commutative Noetherian ring containing a field $\mathbb{K}$, let $I \subseteq A$ be an ideal, let $I=I_{1} \cap \ldots \cap I_{n}$ be its primary decomposition, let $P$ be the poset given by all the possible different sums of the ideals $I_{k}$ s ordered by reverse inclusion, let $T_{[*]}$ be the functor of Setup 4.3, and let $M$ be an $A$-module such that, for any $p \in P, \mathbb{R}^{j} T_{p}(M)=0$ up to a unique value of $j$ (namely, $h_{p}$ ) and such that, for any pair of elements $p<q$, $\operatorname{Hom}_{A}\left(\mathbb{R}^{h_{p}} T_{p}(M), \mathbb{R}^{h_{q}} T_{p}(M)\right)=0$. Then, there is a $\mathbb{K}$-vector space isomorphism

$$
\mathbb{R}^{j} T(M) \cong \bigoplus_{q \in P} \mathbb{R}^{h_{q}} T_{q}(M)^{\oplus m_{j, q}}
$$

where $m_{j, q}=\operatorname{dim}_{\mathbb{K}} \widetilde{H}_{h_{q}-j-1}((q, 1 \widehat{P}) ; \mathbb{K})$.

Mustaţă-Terai's formula boils down to the case where $A=\mathbb{K}\left[x_{1}, \cdots, x_{n}\right]$ is a polynomial ring, $T=\Gamma_{I}$, and $I$ is a Stanley-Reisner ideal.

\subsection{Some additional structures to Hochster-type decompositions}

For the case of Stanley-Reisner rings, Enescu and Hochster [19, 5.1] gave an additional Frobenius structure to Hochster's formula. Moreover, Brun et al. [10] gave a $\mathbb{Z}^{d}$-graded structure to their main decomposition result. Finally, Terai also gave a $\mathbb{Z}^{d}$-graded structure to his formula.

The aim of this section is to extend the decompositions, as $\mathbb{K}$-vector spaces, obtained in Theorems 7.1 and 7.5 to accommodate these extra structures. 


\subsubsection{Additional graded structure}

In order to recover and extend the $\mathbb{Z}^{d}$-graded main result of [10], we need to review first a technical fact; indeed, given a field $\mathbb{K}$ and a group $G$, denote by * ${ }^{*} c_{\mathbb{K}}$ the category of $G$-graded $\mathbb{K}$-vector spaces such that, for any $V \in{ }^{*} \operatorname{vec}_{\mathbb{K}}$, and for any $g \in G, V_{g}$ is a finite-dimensional $\mathbb{K}$-vector space. The below result was proved by Raicu when $\mathbb{K}=\mathbb{C}$ and $G=G_{m}(\mathbb{C}) \times G_{n}(\mathbb{C})$; however, since his proof works verbatim also in this setting, we refer to [54, Lemma 2.8] for details.

Lemma 7.6. ${ }^{*} \operatorname{vec}_{\mathbb{K}}$ is semisimple.

Now, we are ready to establish the main result of this part, whose proof boils down to the fact that any short exact sequence splits in a semisimple category.

Theorem 7.7. Let $\mathbb{K}$ be a field, let $A$ be a commutative Noetherian ring containing $\mathbb{K}$, let $T$ and $\mathcal{T}$ be as in Setup 5.4, and let $P$ be any finite poset. Moreover, assume that $V \in \operatorname{Inv}(\widehat{P}, \mathcal{A})$ is acyclic with respect to the limit, that there is a natural equivalence of functors $\lim _{p \in P} \circ \mathcal{T} \cong T \circ \lim _{p \in P}$, that for any $p \in P, \mathbb{R}^{j} T\left(V_{p}\right)=0$ up to a single value of $j$ (namely, $d_{p}$ ), and that, for any $p \neq q, \operatorname{Hom}_{A}\left(\mathbb{R}^{d_{p}} T\left(V_{p}\right), \mathbb{R}^{d_{q}} T\left(V_{q}\right)\right)=0$. Then, if $\mathcal{B}$ is an abelian, semisimple category containing $\mathcal{A}$ such that $T(\mathcal{B}) \subseteq \mathcal{B}$, and if $V \in \operatorname{Inv}(\widehat{P}, \mathcal{B})$, then there is an isomorphism

$$
\mathbb{R}^{j} T\left(\lim _{p \in P} V_{p}\right) \cong \bigoplus_{q \in P} \mathbb{R}^{d_{q}} T\left(V_{q}\right)^{\oplus M_{j, q}}
$$

in the category $\mathcal{B}$, where $M_{j, q}=\operatorname{dim}_{\mathbb{K}} \widetilde{H}^{j-d_{q}-1}\left(\left(q, 1_{\widehat{P}}\right) ; \mathbb{K}\right)$.

We can also write down the corresponding statement for our Mustaţă-Terai-type formulas; namely the following:

Theorem 7.8. Let $A$ be a commutative Noetherian ring containing a field $\mathbb{k}$, let $I \subseteq A$ be an ideal, let $I=I_{1} \cap \ldots \cap I_{n}$ be its primary decomposition, let $P$ be the poset given by all the possible different sums of the ideals $I_{k}$ 's ordered by reverse inclusion, let $T_{[*]}$ be the functor of Setup 4.3, and let $M$ be an $A$-module such that, for any $p \in P$, $\mathbb{R}^{j} T_{p}(M)=0$ up to a unique value of $j$ (namely, $h_{p}$ ) and such that, for any pair of elements $p<q, \operatorname{Hom}_{A}\left(\mathbb{R}^{h_{p}} T_{p}(M), \mathbb{R}^{h_{q}} T_{p}(M)\right)=0$. Then, if $\mathcal{B}$ is an abelian, semisimple category containing $\mathcal{A}$ such that $T_{p}(\mathcal{B}) \subseteq \mathcal{B}$ for any $p \in P$, and if $M$ is an object of $\mathcal{B}$, then there is 
an isomorphism

$$
\mathbb{R}^{j} T(M) \cong \bigoplus_{q \in P} \mathbb{R}^{h_{q}} T_{q}(M)^{\oplus m_{j, q}}
$$

in the category $\mathcal{B}$, where $m_{j, q}=\operatorname{dim}_{\mathbb{K}} \widetilde{H}_{h_{q-j-1}}\left(\left(q, 1_{\widehat{P}}\right) ; \mathbb{K}\right)$.

As an immediate consequence of Lemma 7.6, Theorem 7.7, and the fact (that we already proved) that the inverse system defining a toric face ring is acyclic with respect to the limit functor, we obtain the following:

Theorem 7.9 (Hochster decomposition for toric face rings). Let $\Sigma \subseteq \mathbb{R}^{d}$ be a rational pointed fan, let $\mathcal{M}_{\Sigma}$ be a Cohen-Macaulay monoidal complex supported on $\Sigma$, let $\mathbb{K}$ be a field, and let $\mathbb{K}\left[\mathcal{M}_{\Sigma}\right]$ be the corresponding toric face ring with $\mathfrak{m}$ as a unique graded maximal ideal. Then, there is an isomorphism of $\mathbb{Z}^{d}$-graded $\mathbb{K}$-vector spaces

$$
H_{\mathfrak{m}}^{i}\left(\mathbb{K}\left[\mathcal{M}_{\Sigma}\right]\right) \cong \bigoplus_{C \in \Sigma} H_{\mathfrak{m}}^{d_{C}}\left(\mathbb{K}\left[M_{C}\right]\right)^{\oplus M_{i, C}}
$$

where $d_{C}:=\operatorname{dim}\left(\mathbb{K}\left[M_{C}\right]\right)$ and $M_{i, C}=\operatorname{dim}_{\mathbb{K}} \widetilde{H}^{i-d_{C}-1}\left(\left(p_{C}, 1_{\widehat{P}}\right) ; \mathbb{K}\right)$.

Remark 7.10. The reader will easily note that Theorem 7.9 recovers and extends [10, 1.3]; it is worth to point out that this formula is still valid under the assumption that $\mathbb{K}\left[\mathcal{M}_{\Sigma}\right]$ is seminormal $[46,4.5]$. However, notice that the seminormal case is not covered by our formalism.

Also as a consequence of Lemma 7.6 and Theorem 7.7, we recover the Takayama's type decomposition for the local cohomology of monomial ideals obtained by Brun and Römer in [9, Corollary 2.3]; namely the following:

Theorem 7.11 (Takayama decomposition for monomial ideals). Let $\Delta$ be a simplicial complex of $d$ vertices, let $\mathbb{K}$ be any field, and let $I \subseteq \mathbb{K}\left[x_{1}, \ldots, x_{d}\right]$ be a monomial ideal such that $\sqrt{I}$ is given by $\Delta$ through the Stanley correspondence. Then, there is an isomorphism of $\mathbb{Z}^{d}$-graded $\mathbb{K}$-vector spaces

$$
H_{\mathfrak{m}}^{i}\left(\mathbb{K}\left[x_{1}, \ldots, x_{d}\right] / I\right) \cong \bigoplus_{F \in \Delta} H_{\mathfrak{m}}^{d_{F}}\left(\mathbb{K}\left[x_{1}, \ldots, x_{d}\right] / I_{p}\right)^{\oplus M_{i, F}}
$$

where $F \subset \Delta$ is the face determined by $\sqrt{I_{p}}, d_{F}:=\operatorname{dim}\left(\mathbb{K}\left[x_{1}, \ldots, x_{d}\right] / I_{p}\right)$, and finally, $M_{i, F}=\operatorname{dim}_{\mathbb{K}} \widetilde{H}^{i-d_{F}-1}\left(\left(p_{F}, 1_{\widehat{P}}\right) ; \mathbb{K}\right)$. 
Proof. It is known [26, Theorem 1.3.1 and Corollary 1.3.2] that any monomial ideal admits a minimal primary decomposition made up by ideals generated by pure powers of the variables; so, let $I=I_{1} \cap \ldots \cap I_{n}$ be such a minimal primary decomposition, and consider $P$ as the poset attached to it. Then it is clear that, for each $p \in P, A / I_{p}$ is a Cohen-Macaulay ring and that, for each $p \neq q, I_{q}$ is not contained in any minimal prime of $I_{p}$. Therefore, our claimed decomposition follows immediately combining Theorem 5.22 jointly with Lemma 7.6 and Theorem 7.7.

We can also obtain a Hochster-type decomposition for the local cohomology modules attached to some affine, central subspace arrangements of linear varieties over a field $\mathbb{k}$; namely the following:

Theorem 7.12 (Hochster decompostion for some central arrangements). Let $\mathbb{K}$ be a field, and let $I \subseteq \mathbb{K}\left[x_{1}, \ldots, x_{d}\right]$ be the vanishing ideal of a central arrangement of linear varieties in $\mathbb{K}^{d}$. Moreover, let $P$ be the poset given by all the possible nonempty intersections of subarrangements ordered by inclusion; for each $p \in P$, denote by $I_{p}$ the corresponding vanishing ideal, and suppose that the inverse system $\left(\mathbb{K}\left[x_{1}, \ldots, x_{d}\right] / I_{p}\right)_{p \in P}$ is acyclic with respect to the limit. Then, there is an isomorphism of $\mathbb{Z}$-graded $\mathbb{K}$-vector spaces

$$
H_{\mathfrak{m}}^{i}\left(\lim _{p \in P} \mathbb{K}\left[x_{1}, \ldots, x_{d}\right] / I_{p}\right) \cong \bigoplus_{p \in P} H_{\mathfrak{m}}^{d_{p}}\left(\mathbb{K}\left[x_{1}, \ldots, x_{d}\right] / I_{p}\right)^{\oplus M_{i, p}}
$$

where $d_{p}=\operatorname{dim}\left(\mathbb{K}\left[x_{1}, \ldots, x_{d}\right] / I_{p}\right)$ and $M_{i, p}=\operatorname{dim}_{\mathbb{K}} \widetilde{H}^{i-d_{p}-1}\left(\left(p, 1_{\widehat{P}}\right) ; \mathbb{K}\right)$. If, in addition, there is an isomorphism

$$
\lim _{p \in P} \mathbb{K}\left[x_{1}, \ldots, x_{d}\right] / I_{p} \cong \mathbb{K}\left[x_{1}, \ldots, x_{d}\right] / I
$$

(see Remarks 5.2 and 5.3), then

$$
H_{\mathfrak{m}}^{i}\left(\mathbb{K}\left[x_{1}, \ldots, x_{d}\right] / I\right) \cong \bigoplus_{p \in P} H_{\mathfrak{m}}^{d_{p}}\left(\mathbb{K}\left[x_{1}, \ldots, x_{d}\right] / I_{p}\right)^{\oplus M_{i, p}}
$$

In case of central arrangements, we can deduce immediately from Theorem 7.12 the corresponding $\mathbb{Z}$-graded Hilbert series; namely the following: 
Theorem 7.13 (Hilbert series of local cohomology for some central arrangements). Preserving the assumptions and notations of Theorem 7.12, one has that

$$
H\left(H_{\mathfrak{m}}^{i}\left(\lim _{p \in P} \mathbb{K}\left[x_{1}, \ldots, x_{d}\right] / I_{p}\right) ; t\right)=\sum_{p \in P} \frac{\operatorname{dim}_{\mathbb{K}} \widetilde{H}^{i-d_{p}-1}\left(\left(p, 1_{\widehat{P}}\right) ; \mathbb{K}\right)}{(t-1)^{d_{p}}},
$$

where $H(-; t)$ denotes the $\mathbb{Z}$-graded Hilbert series. If, in addition, there is an isomorphism

$$
\lim _{p \in P} \mathbb{K}\left[x_{1}, \ldots, x_{d}\right] / I_{p} \cong \mathbb{K}\left[x_{1}, \ldots, x_{d}\right] / I
$$

(see Remarks 5.2 and 5.3), then

$$
H\left(H_{\mathfrak{m}}^{i}\left(\mathbb{K}\left[x_{1}, \ldots, x_{d}\right] / I\right) ; t\right)=\sum_{p \in P} \frac{\operatorname{dim}_{\mathbb{K}} \widetilde{H}^{i-d_{p}-1}\left(\left(p, 1_{\widehat{P}}\right) ; \mathbb{K}\right)}{(t-1)^{d_{p}}} .
$$

Proof. The additivity of Hilbert series on short exact sequences, combined with Theorem 7.12, implies that

$$
H\left(H_{\mathfrak{m}}^{i}\left(\lim _{p \in P} \mathbb{K}\left[x_{1}, \ldots, x_{d}\right] / I_{p}\right) ; t\right)=\sum_{p \in P} \operatorname{dim}_{\mathbb{K}} \widetilde{H}^{i-d_{p}-1}\left(\left(p, 1_{\widehat{P}}\right) ; \mathbb{K}\right) H\left(H_{\mathfrak{m}}^{d_{p}}\left(\mathbb{K}\left[x_{1}, \ldots, x_{d}\right] / I_{p}\right)\right),
$$

so it is enough to calculate $H\left(H_{\mathfrak{m}}^{d_{p}}\left(\mathbb{K}\left[x_{1}, \ldots, x_{d}\right] / I_{p}\right)\right)$; indeed, fix $p \in P$. Since $I_{p}$ is a prime ideal generated by $\mathbb{K}$-linearly independent linear forms, it is straightforward to check that

$$
H\left(H_{\mathfrak{m}}^{d_{p}}\left(\mathbb{K}\left[x_{1}, \ldots, x_{d}\right] / I_{p}\right)\right)=\left(\frac{1 / t}{1-1 / t}\right)^{d_{p}}=\frac{1}{(t-1)^{d_{p}}}
$$

just what we finally wanted to prove.

Finally, we can also write down our Mustaţă-Terai-type formula for certain toric face rings; the statement is as follows:

Theorem 7.14 (Mustaţă-Terai decomposition for toric face rings). Let $\Sigma \subseteq \mathbb{R}^{d}$ be a rational pointed fan, let $\mathcal{M}_{\Sigma}$ be a Cohen-Macaulay monoidal complex supported on $\Sigma$, let $\mathbb{K}$ be a field of prime characteristic, and let $\mathbb{K}\left[\mathcal{M}_{\Sigma}\right]$ be the corresponding toric face ring with $I=I_{\Sigma}$ as its defining ideal inside the polynomial ring $A=\mathbb{K}\left[x_{1}, \ldots, x_{d}\right]$. Then, there is an isomorphism of $\mathbb{Z}^{d}$-graded $\mathbb{K}$-vector spaces

$$
H_{I}^{i}(A) \cong \bigoplus_{C \in \Sigma} H_{I_{C}}^{h_{C}}(A)^{\oplus m_{i, C}},
$$


where $h_{C}=d-d_{C}, d_{C}:=\operatorname{dim}\left(\mathbb{K}\left[M_{C}\right]\right)$, and $m_{i, C}=\operatorname{dim}_{\mathbb{K}} \widetilde{H}_{h_{C}-i-1}\left(\left(p_{C}, 1_{\widehat{P}}\right) ; \mathbb{K}\right)$.

\subsubsection{Application to the regularity of some central arrangements}

The goal of this part is to use the decomposition obtained in Theorem 7.12 to calculate the (Castelnuovo-Mumford) regularity of certain arrangement of linear varieties (see Theorem 7.18); this leads to an alternative proof, specific for this kind of arrangements, of the so-called Subspace Arrangements Theorem (see Theorem 7.19), originally obtained by Derksen and Sidman in [15, Theorem 2.1].

Before doing so, we need to prove some auxiliary results; next lemma is an immediate consequence of Remark 3.6 and its proof is left to the interested reader.

Lemma 7.15. Preserving the assumptions and notations of Theorem 7.12, let $n$ be the number of irreducible components of the arrangement. Then, the following assertions hold.

(i) For any $p \in P, \operatorname{rank}\left(\left(p, 1_{\widehat{P}}\right)\right) \leq n-1$.

(ii) Given $p \in P$ and $i \geq 0$ such that $i-d_{p}-1 \geq n, \widetilde{H}^{i-d_{p}-1}\left(\left(p, 1_{\widehat{P}}\right) ; \mathbb{K}\right)=0$.

We also need to review the following notion of $\mathbb{Z}$-graded vector spaces.

Definition 7.16. Let $k$ be any field, and let $E$ be a $\mathbb{Z}$-graded $k$-vector space; set

$$
\operatorname{end}(E):=\max \left\{j \in \mathbb{Z}: E_{j} \neq 0\right\} \text {. }
$$

The last preliminary result we need is the behavior of the end of a $\mathbb{Z}$-graded vector space with respect to finite direct sums; the proof is left to the interested reader.

Lemma 7.17. Let $I$ be a finite index set, let $k$ be any field, let $\left\{E_{i}\right\}_{i \in I}$ be a family of $\mathbb{Z}$-graded $k$-vector spaces, and set

$$
E:=\bigoplus_{i \in I} E_{i}
$$

Then, $\operatorname{end}(E)=\sup _{i \in I}\left\{\operatorname{end}\left(E_{i}\right)\right\}$.

Now, we are in a position to state our main result about the regularity of arrangements of linear varieties, which is the below:

Theorem 7.18 (Regularity of an arrangement of linear varieties). Let $\mathbb{K}$ be a field, and let $I \subseteq \mathbb{K}\left[x_{1}, \ldots, x_{d}\right]$ be the vanishing ideal of a central arrangement of linear varieties in $\mathbb{K}^{d}$. Moreover, let $P$ be the poset given by all the possible nonempty intersections of subarrangements ordered by inclusion; for each $p \in P$, denote by $I_{p}$ the corresponding vanishing ideal, and suppose that the inverse system $\left(\mathbb{K}\left[x_{1}, \ldots, x_{d}\right] / I_{p}\right)_{p \in P}$ 
is acyclic with respect to the limit. Then,

$$
\operatorname{reg}\left(\lim _{p \in P} \mathbb{K}\left[x_{1}, \ldots, x_{d}\right] / I_{p}\right)=\max _{i \geq 0, p \in P}\left\{i-d_{p}: M_{i, p} \neq 0\right\},
$$

where $d_{p}=\operatorname{dim}\left(\mathbb{K}\left[x_{1}, \ldots, x_{d}\right] / I_{p}\right)$ and $M_{i, p}=\operatorname{dim}_{\mathbb{K}} \widetilde{H}^{i-d_{p^{-1}}}\left(\left(p, 1_{\widehat{P}}\right) ; \mathbb{K}\right)$.

Proof. It is known [17, pages 58-59] that

$$
\operatorname{reg}\left(\lim _{p \in P} \mathbb{K}\left[x_{1}, \ldots, x_{d}\right] / I_{p}\right)=\max _{i \geq 0}\left\{\text { end }\left(H_{\mathfrak{m}}^{i}\left(\lim _{p \in P} \mathbb{K}\left[x_{1}, \ldots, x_{d}\right] / I_{p}\right)\right)+i\right\}
$$

In this way, combining Theorem 7.12 jointly with Lemma 7.17 it follows that

$$
\operatorname{reg}\left(\lim _{p \in P} \mathbb{K}\left[x_{1}, \ldots, x_{d}\right] / I_{p}\right)=\max _{i \geq 0, p \in P}\left\{\operatorname{end}\left(H_{\mathfrak{m}}^{d_{p}}\left(\mathbb{K}\left[x_{1}, \ldots, x_{d}\right] / I_{p}\right)\right)+i: M_{i, p} \neq 0\right\}
$$

On the other hand, given any $p \in P,[21$, Remark 3.1.6] implies that

$$
\text { end }\left(H_{\mathfrak{m}}^{d_{p}}\left(\mathbb{K}\left[x_{1}, \ldots, x_{d}\right] / I_{p}\right)\right)=-d_{p}
$$

Summing up, combining all the foregoing equalities one finally obtains that

$$
\operatorname{reg}\left(\lim _{p \in P} \mathbb{K}\left[x_{1}, \ldots, x_{d}\right] / I_{p}\right)=\max _{i \geq 0, p \in P}\left\{i-d_{p}: M_{i, p} \neq 0\right\},
$$

and the proof is therefore completed.

Remark 7.19. As an immediate consequence of Theorem 7.18 and Lemma 7.15, we obtain a new proof, specific for arrangements satisfying $A / I \cong \lim _{p \in P} A / I_{p}$ and that $A /[*]$ is acyclic with respect to the limit, of the so-called Subspace Arrangements Theorem, originally proved by Derksen and Sidman in [15, Theorem 2.1] (see also [17, Theorem 4.19]) for any central arrangement. This reflects the fact that, in general, the regularity of an algebraic variety is not determined by its intersection lattice.

\subsubsection{Additional Frobenius structure}

Finally, we are ready to provide the announced generalization of $[19,5.1]$; namely the following: 
Theorem 7.20. Under the assumptions of Theorem 7.9, if $\mathbb{K}$ is a field of prime characteristic $p$, there is a $\mathbb{K}[\Theta ; F]$-isomorphism

$$
H_{\mathfrak{m}}^{i}\left(\mathbb{K}\left[\mathcal{M}_{\Sigma}\right]\right) \cong \bigoplus_{C \in \Sigma} H_{\mathfrak{m}}^{d_{C}}\left(\mathbb{K}\left[M_{C}\right]\right)^{\oplus M_{i, C}}
$$

Moreover, if $\mathcal{M}_{\Sigma}$ is $F$-rational, then every $H_{\mathfrak{m}}^{i}\left(\mathbb{K}\left[\mathcal{M}_{\Sigma}\right]\right)$ is a finite direct sum of simple $A[\Theta ; F]$-modules (where $A=\mathbb{K}\left[\mathcal{M}_{\Sigma}\right]$ ) on which $F$ acts injectively. In particular (cf. [19, Theorem 5.1]), if $\left(A_{1}, \mathfrak{m}_{1}\right)$ is either $\mathbb{K}\left[\mathcal{M}_{\Sigma}\right]_{\mathfrak{m}}$ or its completion, then $H_{\mathfrak{m}}^{i}\left(\mathbb{K}\left[\mathcal{M}_{\Sigma}\right]\right)$ can be identified with $H_{\mathfrak{m}_{1}}^{i}\left(A_{1}\right)$, and $H_{\mathfrak{m}_{1}}^{i}\left(A_{1}\right)$ is a finite direct sum of simple $A_{1}[\Theta ; F]$-modules on which $F$ acts injectively, and therefore it has only a finite number of $F$-compatible submodules because of [19, Theorem 4.12].

Proof. By Example 5.3, the spectral sequence (respectively, the corresponding filtration produced by its degeneration) can be regarded as spectral sequence (respectively, filtration) in the category of left $\mathbb{F}_{p}[\Theta ; F]$-modules; moreover, it is also clear (by means of Theorem 7.1) that the claimed decomposition holds in the category of $\mathbb{K}$-vector spaces. In this way, it only remains to check that the Frobenius map preserves the decomposition; in other words, that at both sides of such decomposition the Frobenius acts in exactly the same way. The reader will easily note that, hereafter, we follow so closely the argument pointed out during the proof of $[19,5.1]$.

The 1st thing one has to ensure is that the action of $F$ is $\mathbb{K}$-linear (otherwise, compatibility with the $\mathbb{K}$-vector space structure would be impossible); with this purpose in mind, we have to restrict our ground field of coefficients to $\mathbb{F}_{p}$ (here, we are using Fermat's Little Theorem). Notice that we can do so without loss of generality; indeed, firstly, the multiplicities $m_{i, c}$ 's appearing in the decomposition are not affected by this restriction of coefficients. Secondly, the action of $F$ on $H_{\mathfrak{m}}^{i}\left(\mathbb{K}\left[\mathcal{M}_{\Sigma}\right]\right)$ can be canonically identified with the one induced on the cohomology of the complex $L^{\bullet}\left(\mathcal{M}_{\Sigma} ; \mathbb{K}\right)$ (labeled $L^{\bullet}\left(\mathcal{M}_{\Sigma}\right)$ in [28, p. 256], see also [28, Theorem 3.2]), and this action on $L^{\bullet}\left(\mathcal{M}_{\Sigma} ; \mathbb{K}\right)$ is obtained from the action on $L^{\bullet}\left(\mathcal{M}_{\Sigma} ; \mathbb{F}_{p}\right)$ by applying $\mathbb{K} \otimes_{\mathbb{F}_{p}}(-)$. Finally, for each cone $C \in \Sigma$, the action of $F$ on $H_{\mathfrak{m}}^{i}\left(\mathbb{K}\left[M_{C}\right]\right)$ can be canonically identified with the one induced on the cohomology of the complex $L^{\bullet}(\mathbb{K})$ (labeled $L^{\bullet}$ in [11, p. 267], see also [11, Theorem 6.2.5]), and this action on $L^{\bullet}(\mathbb{K})$ is obtained from the action on $L^{\bullet}\left(\mathbb{F}_{p}\right)$ by applying $\mathbb{K} \otimes_{\mathbb{F}_{p}}(-)$.

Summing up, we can suppose that $\mathbb{K}=\mathbb{F}_{p}$; under this assumption, the value of the action of $F$ on both sides of our decomposition is the one that acts on a coset of the form $a \eta(a \in \mathbb{K})$ by $F(a \eta)=a \eta^{p}$, which is what we want to check. 
Finally, if $\mathcal{M}_{\Sigma}$ is $F$-rational, then for each cone $C \in \Sigma, H_{\mathfrak{m}}^{\operatorname{dim}(C)}\left(\mathbb{K}\left[M_{C}\right]\right)$ is a simple $A[\Theta ; F]$-module $[58,2.6]$; the proof is therefore completed.

Remark 7.21. Notice that the assumption of $F$-rationality on $\mathcal{M}_{\Sigma}$ required in Theorem 7.20 holds when, for instance, $\mathcal{M}_{\Sigma}$ is a normal monoidal complex; indeed, under this assumption, since for each cone $C \in \Sigma, \mathbb{K}\left[M_{C}\right]$ is a normal affine monoid ring, one has that $\mathbb{K}\left[M_{C}\right]$ is a direct summand of a Laurent polynomial ring [11, Exercise 6.1.10] and therefore it is $F$-regular by [27, Proposition 4.12]. This shows, in particular, that Theorem 7.20 extends $[19,5.1]$ to Stanley toric face rings.

\section{Extension Problems for Cohomological Spectral Sequences: A Gräbe's Type Formula}

In the spirit of [5, Section 3], the aim of this section is to focus on the study of the extension problems attached to the corresponding filtrations produced by the degeneration of our previously introduced homological spectral sequences.

\subsection{Extension problems for homological spectral sequences associated to modules}

Consider the homological spectral sequence

$$
E_{2}^{-i, j}=\mathbb{L}_{i} \operatorname{colim}_{p \in P} \mathbb{R}^{j} T_{[*]}(M) \underset{i}{\longrightarrow} \mathbb{R}^{j-i} T(M)
$$

constructed in Section 4. In Theorem 4.11 we provided necessary conditions for the spectral sequence to degenerate at the $E_{2}$-page. Therefore, by Corollary 4.12 , we obtain the following collection of short exact sequences (where $b \in \mathbb{N}$ ):

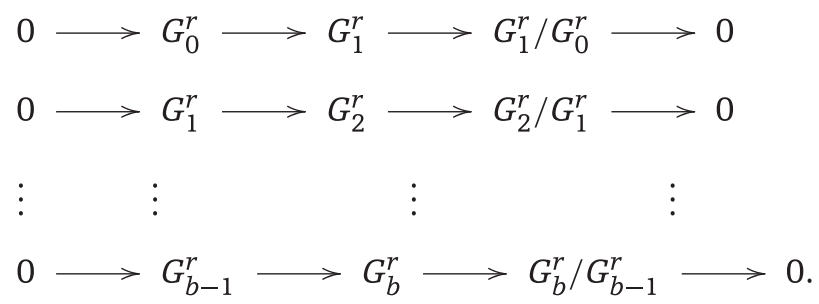

where

$$
G_{k}^{r} / G_{k-1}^{r} \cong \bigoplus_{\left\{q \in P \mid k+r=h_{q}\right\}} \mathbb{R}^{h_{q}} T_{q}(M)^{\oplus m_{k, q}}
$$


where $m_{k, q}:=\operatorname{dim}_{\mathbb{K}}\left(\widetilde{H}_{k-1}\left(\left(q, 1_{\widehat{P}}\right) ; \mathbb{K}\right)\right)$. Hereafter, we omit the superscript $r$; moreover, we have to point out that, for any $k$,

$$
\left(s_{k}\right): \quad 0 \longrightarrow G_{k-1} \longrightarrow G_{k} \longrightarrow G_{k} / G_{k-1} \longrightarrow 0
$$

may be regarded as an element of $\operatorname{Ext}_{\mathcal{A}}^{1}\left(G_{k} / G_{k-1}, G_{k-1}\right)$. In order to study the corresponding extension problems we may use the following mild generalization of [5, Lemma of p.47].

Lemma 8.1. We assume, in addition, that $\operatorname{Ext}_{\mathcal{A}}^{1}\left(\mathbb{R}^{h_{p}} T_{p}(M), \mathbb{R}^{h_{q}} T_{q}(M)\right)=0$ provided $h_{p} \geq$ $h_{q}+2$. Then, the natural maps $\operatorname{Ext}_{\mathcal{A}}^{1}\left(G_{k} / G_{k-1}, G_{k-1}\right) \longrightarrow \operatorname{Ext}_{\mathcal{A}}^{1}\left(G_{k} / G_{k-1}, G_{k-1} / G_{k-2}\right)$ are injective for all $k \geq 2$.

Proof. Consider the short exact sequence

$$
\left(s_{k-1}\right): 0 \longrightarrow G_{k-2} \longrightarrow G_{k-1} \longrightarrow G_{k-1} / G_{k-2} \longrightarrow 0 \text {. }
$$

In this way, applying the functor $\operatorname{Hom}_{\mathcal{A}}\left(G_{k} / G_{k-1},-\right)$ to $\left(s_{k-1}\right)$, one obtains the following exact sequence: $\operatorname{Ext}_{\mathcal{A}}^{1}\left(G_{k} / G_{k-1}, G_{k-2}\right) \longrightarrow \operatorname{Ext}_{\mathcal{A}}^{1}\left(G_{k} / G_{k-1}, G_{k-1}\right) \longrightarrow \operatorname{Ext}_{\mathcal{A}}^{1}\left(G_{k} / G_{k-1}, G_{k-1} /\right.$ $\left.G_{k-2}\right)$. So, applying once again $\operatorname{Hom}_{\mathcal{A}}\left(G_{k} / G_{k-1},-\right)$ to the short exact sequence ( $s_{l}$ ) for $l \leq k-2$ and descending induction, it turns out that we only need to check that $\operatorname{Ext}_{\mathcal{A}}^{1}\left(G_{k} / G_{k-1}, G_{l} / G_{l-1}\right)=0$ for any $l \leq k-2$. However, applying Corollary 4.12 it is enough to show that the group $\operatorname{Ext}_{\mathcal{A}}^{1}\left(\mathbb{R}^{h_{p}} T_{p}(M), \mathbb{R}^{h_{q}} T_{q}(M)\right)$ vanishes, where $h_{q} \leq j-2$ and $h_{p}=j$. But this vanishing holds by assumption.

\subsubsection{Mayer-Vietoris spectral sequence of local cohomology modules}

These extension problems were studied in [5] for the spectral sequence

$$
E_{2}^{-i, j}=\mathbb{L}_{i} \operatorname{colim}_{p \in P} H_{I_{p}}^{j}(A) \underset{i}{\Longrightarrow} H_{I}^{j-i}(A),
$$

where $A=\mathbb{K}\left[x_{1}, \cdots, x_{d}\right]$ is the polynomial ring over a field $\mathbb{K}$ and $I$ is a squarefree

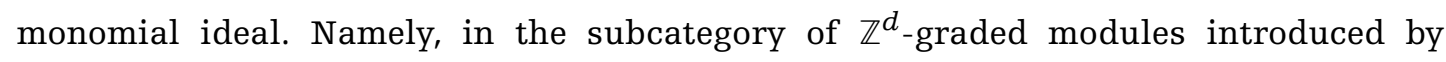
Yanagawa [64] under the notion of straight modules, these extension problems are nontrivial and are described by the multiplication by the variables $x_{i}$. In particular this result recovers the description given by Mustaţă in [42].

We point out that, whenever our field $\mathbb{K}$ is of characteristic 0 , the category of straight modules is equivalent to the category of regular holonomic $D$-modules 
with variation 0 [5, Section 4], so these extension problems are also nontrivial in this subcategory of $D$-modules. In this way, it is natural to ask whether these extension problems are trivial in the category of $D$-modules; however, this is not the case, as shown using Lyubeznik numbers introduced in [35].

Example 8.2. Let $I:=(x, y z) \subset A:=\mathbb{K}[x, y, z]$, where $\mathbb{K}$ is any field. In this case, if

$$
0 \longrightarrow H_{(x, Y)}^{2}(A) \oplus H_{(x, Z)}^{2}(A) \longrightarrow H_{I}^{2}(A) \longrightarrow H_{(x, Y, Z)}^{3}(A) \longrightarrow 0,
$$

was split, then one would obtain the following equality of Lyubeznik numbers:

$$
\lambda_{1,1}(A / I)=1 \neq 2=\lambda_{1,1}\left(\frac{\mathbb{K}[x, y, z]}{(x, y)}\right)+\lambda_{1,1}\left(\frac{\mathbb{K}[x, y, z]}{(x, z)}\right)+\lambda_{1,0}\left(\frac{\mathbb{K}[x, y, z]}{(x, y, z)}\right),
$$

which is clearly false [4].

Remark 8.3. The above example also shows that, when $\mathbb{K}$ is a field of prime characteristic, these extension problems are also nontrivial in the category of $F$-modules, because any $F$-module is, in particular, a $D$-module (see [36, Section 5] for details).

\subsection{Extension problems for cohomological spectral sequences associated to inverse systems}

Our next goal is to carry out a similar business with the filtration produced in Theorem 5.18; indeed, under its assumptions Theorem 5.18 provides a spectral sequence

$$
E_{2}^{i, j}=\bigoplus_{j=d_{q}} \mathbb{R}^{d_{q}} T\left(V_{q}\right)^{\oplus M_{i, q}} \underset{i}{\Longrightarrow} \mathbb{R}^{i+j} T\left(\lim _{p \in P} V_{p}\right)
$$

where $M_{i, q}=\operatorname{dim}_{\mathbb{K}} \widetilde{H}^{i-d_{q}-1}\left(\left(q, 1_{\widehat{P}}\right) ; \mathbb{K}\right)$. Moreover, we have a collection of short exact sequences (for some $b \in \mathbb{N}$ )

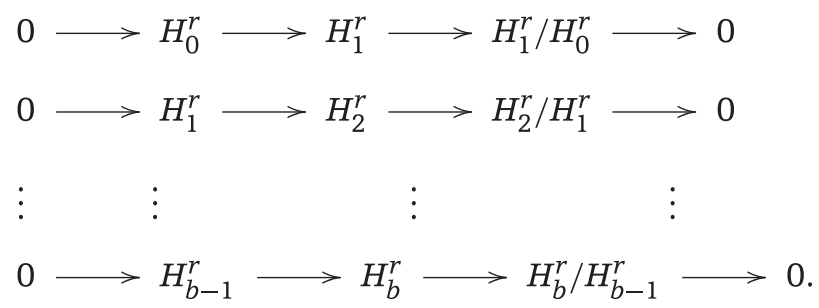


where the quotients $H_{k}^{r} / H_{k-1}^{r}$ can be decomposed in the following manner:

$$
H_{k}^{r} / H_{k-1}^{r} \cong \bigoplus_{\left\{q \in P \mid r-k=d_{q}\right\}} \mathbb{R}^{d_{q}} T\left(V_{q}\right)^{\oplus M_{k, q}}
$$

where $M_{k, q}:=\operatorname{dim}_{\mathbb{K}}\left(\widetilde{H}^{k-d_{q}-1}\left(\left(q, 1_{\widehat{P}}\right) ; \mathbb{K}\right)\right)$. From now on, we omit the superscript $r$; in addition, the reader should point out that, for each $k$,

$$
\left(h_{k}\right): 0 \longrightarrow H_{k-1} \longrightarrow H_{k} \longrightarrow H_{k} / H_{k-1} \longrightarrow 0
$$

can be considered as a member of $\operatorname{Ext}_{\mathcal{A}}^{1}\left(H_{k} / H_{k-1}, H_{k-1}\right)$. The next result is just a reformulation of Lemma 8.1 in this setup; since its proof is exactly the same as the one of Lemma 8.1, we omit the details.

Lemma 8.4. We assume, in addition, that $\operatorname{Ext}_{\mathcal{A}}^{1}\left(\mathbb{R}^{d_{p}} T\left(V_{p}\right), \mathbb{R}^{d_{q}} T\left(V_{q}\right)\right)=0$ provided $d_{p} \geq d_{q}+2$. Then, the natural maps $\operatorname{Ext}_{\mathcal{A}}^{1}\left(H_{k} / H_{k-1}, H_{k-1}\right) \longrightarrow \operatorname{Ext}_{\mathcal{A}}^{1}\left(H_{k} / H_{k-1}, H_{k-1} / H_{k-2}\right)$ are injective for all $k \geq 2$.

\subsubsection{Another spectral sequence of local cohomology modules}

From now on we will consider the spectral sequence

$$
E_{2}^{i, j}=\bigoplus_{j=d_{q}} H_{\mathfrak{m}}^{\operatorname{dim}\left(A / I_{q}\right)}\left(A / I_{q}\right)^{\oplus M_{i, q}} \Longrightarrow H_{\mathfrak{m}}^{i+j}\left(\lim _{p \in P} A / I_{p}\right)
$$

considered in Theorem 5.22, degenerating at its $E_{2}$-sheet. In this situation, the Ext group considered in Lemma 8.4 can be decomposed into the following way:

$$
\operatorname{Ext}_{\mathcal{A}}^{1}\left(H_{k} / H_{k-1}, H_{k-1} / H_{k-2}\right) \cong \bigoplus_{\substack{r-k=\operatorname{dim}\left(A / I_{p}\right) \\ r-k-1=\operatorname{dim}\left(A / I_{q}\right)}} \operatorname{Ext}_{\mathcal{A}}^{1}\left(H_{\mathfrak{m}}^{\operatorname{dim}\left(A / I_{p}\right)}\left(A / I_{p}\right), H_{\mathfrak{m}}^{\operatorname{dim}\left(A / I_{q}\right)}\left(A / I_{q}\right)\right)
$$

Keeping in mind this decomposition, it seems to us that it can be of some interest to calculate Ext groups of the form $\operatorname{Ext}_{A}^{1}\left(H_{\mathfrak{m}}^{d-t}\left(A / I_{t}\right), H_{\mathfrak{m}}^{d-t-1}\left(A / I_{t+1}\right)\right)$, where the ideal $I_{t}$ $(1 \leq t \leq d-1)$ is generated by an $A$-regular sequence; this will be our next goal. Indeed, we shall consider two different cases: when $A=\mathbb{K} \llbracket x_{1}, \ldots, x_{d} \rrbracket$ (we refer to this situation as the local case) and the case where $A=\mathbb{K}\left[x_{1}, \ldots, x_{d}\right]$ graded in a certain way, which we specify more precisely later on (we refer to this situation as the graded case). 
Before doing so, we show that, in general, the extension problems associated to the filtration produced by Theorem 5.22 are nontrivial in the category of $A$-modules; we do so by means of the below:

Example 8.5. Let $\mathbb{K}$ be any field, and let $I:=(x, y z) \subset \mathbb{K}[x, y, z]_{(x, y, z)}=: A$; in this case, the filtration produced by Theorem 5.22 boils down to the following short exact sequence, where $\mathfrak{m}$ denotes the maximal ideal of $A$ :

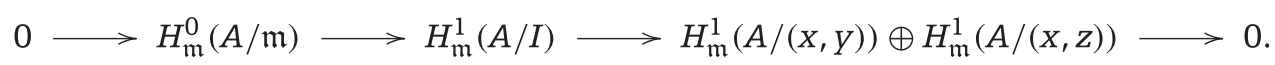

So, if the short exact sequence was split, then $\operatorname{Att}\left(H_{\mathfrak{m}}^{0}(A / \mathfrak{m})\right) \subseteq \operatorname{Att}\left(H_{\mathfrak{m}}^{1}(A / I)\right)$ by [8, 7.2.6]; however, this inclusion is false, because $[8,7.3 .2] \operatorname{implies}$ that $\operatorname{Att}\left(H_{\mathfrak{m}}^{0}(A / \mathfrak{m})\right)=\{\mathfrak{m}\}$ and $\operatorname{Att}\left(H_{\mathfrak{m}}^{1}(A / I)\right)=\{(x, y),(x, z)\}$.

\subsubsection{The local case}

The main result of this part is the following:

Proposition 8.6. Let $\mathbb{K}$ be any field, set $A:=\mathbb{K} \llbracket x_{1}, \ldots, x_{d} \rrbracket$ and let $y_{1}, \ldots, y_{n}$ be an $A$ regular sequence; moreover, for each $1 \leq t \leq n$, set $I_{t}:=\left\langle y_{1}, \ldots, y_{t}\right\rangle$ and $Q_{t}:=H_{\mathfrak{m}}^{d-t}\left(A / I_{t}\right)$. Finally, $(-)^{\vee}$ denotes the Matlis duality functor $\operatorname{Hom}_{A}(-, E)$, where $E$ denotes a choice of injective hull of $\mathbb{K}$ over $A$. Then, the following statements hold:

(i) $\operatorname{Hom}_{A}\left(A / I_{t+1}, A / I_{t}\right)=0$.

(ii) $\operatorname{Hom}_{A}\left(A / I_{t}, A / I_{t}\right)=A / I_{t}$.

(iii) $\operatorname{Ext}_{A}^{1}\left(A / I_{t+1}, A / I_{t}\right)=A / I_{t+1}$; more precisely, $\operatorname{Ext}_{A}^{1}\left(A / I_{t+1}, A / I_{t}\right)$ is a free $\left(A / I_{t+1}\right)$-module of rank 1 that admits as generator the class of the short exact sequence

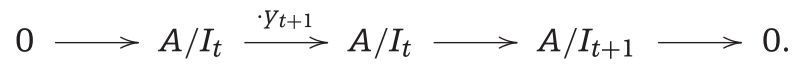

(iv) $\operatorname{Ext}_{A}^{1}\left(\left(0:_{E} I_{t}\right),\left(0:_{E} I_{t+1}\right)\right)=A / I_{t+1}$.

(v) $\operatorname{Ext}_{A}^{1}\left(Q_{t}, Q_{t+1}\right)=A / I_{t+1}$.

Proof. Since $y_{1}, \ldots, y_{n}$ form an $A$-regular sequence, for each $1 \leq t \leq n$, one has $\left(I_{t}:_{A}\right.$ $\left.I_{t+1}\right)=I_{t}$, whence

$$
\operatorname{Hom}_{A}\left(A / I_{t+1}, A / I_{t}\right) \cong \frac{\left(I_{t}:_{A} I_{t+1}\right)}{I_{t}}=0,
$$

just what we firstly wanted to check. 
On the other hand, consider the short exact sequence

$$
0 \longrightarrow A / I_{t} \stackrel{\cdot y_{t+1}}{\longrightarrow} A / I_{t} \longrightarrow A / I_{t+1} \longrightarrow 0
$$

In this way, applying to this short exact sequence the functor $\operatorname{Hom}_{A}\left(-, A / I_{t}\right)$ one obtains the following exact one:

$$
\begin{array}{r}
0 \longrightarrow \operatorname{Hom}_{A}\left(A / I_{t+1}, A / I_{t}\right) \longrightarrow \operatorname{Hom}_{A}\left(A / I_{t}, A / I_{t}\right) \stackrel{\cdot y t+1}{\longrightarrow} \operatorname{Hom}_{A}\left(A / I_{t}, A / I_{t}\right) \\
\longrightarrow \operatorname{Ext}_{A}^{1}\left(A / I_{t+1}, A / I_{t}\right) \longrightarrow \operatorname{Ext}_{A}^{1}\left(A / I_{t}, A / I_{t}\right) \stackrel{\cdot y t+1}{\longrightarrow} \operatorname{Ext}_{A}^{1}\left(A / I_{t}, A / I_{t}\right) .
\end{array}
$$

Thus, since

$$
\operatorname{Hom}_{A}\left(A / I_{t}, A / I_{t}\right) \cong \frac{\left(I_{t}:_{A} I_{t}\right)}{I_{t}}=A / I_{t}
$$

and $\operatorname{Hom}_{A}\left(A / I_{t+1}, A / I_{t}\right)=0$ one can rewrite the previous exact sequence in the following way:

$$
0 \longrightarrow A / I_{t} \stackrel{\cdot y_{t+1}}{\longrightarrow} A / I_{t} \longrightarrow \operatorname{Ext}_{A}^{1}\left(A / I_{t+1}, A / I_{t}\right) \longrightarrow \operatorname{Ext}_{A}^{1}\left(A / I_{t}, A / I_{t}\right) \stackrel{\cdot y_{t+1}}{\longrightarrow} \operatorname{Ext}_{A}^{1}\left(A / I_{t}, A / I_{t}\right) .
$$

Therefore, since the cokernel of $A / I_{t} \stackrel{\cdot{ }^{t+1}}{\longrightarrow} A / I_{t}$ is $A / I_{t+1}$ one obtains the following:

$$
0 \longrightarrow A / I_{t+1} \longrightarrow \operatorname{Ext}_{A}^{1}\left(A / I_{t+1}, A / I_{t}\right) \longrightarrow \operatorname{Ext}_{A}^{1}\left(A / I_{t}, A / I_{t}\right) \stackrel{\cdot y_{t+1}}{\longrightarrow} \operatorname{Ext}_{A}^{1}\left(A / I_{t}, A / I_{t}\right)
$$

Now, we claim that $\operatorname{Ext}_{A}^{1}\left(A / I_{t}, A / I_{t}\right)=\left(A / I_{t}\right)^{\oplus t}$; indeed, $\operatorname{Ext}_{A}^{1}\left(A / I_{t}, A / I_{t}\right)$ can be computed as $H^{1}\left(\operatorname{Hom}_{A}\left(K_{\bullet}\left(y_{1}, \ldots, y_{t}\right), A / I_{t}\right)\right)$, that is, the 1st cohomology group of the cochain complex obtained by applying the functor $\operatorname{Hom}_{A}\left(-, A / I_{t}\right)$ to the Koszul resolution $K_{\bullet}\left(y_{1}, \ldots, y_{t}\right)$ of $A / I_{t}$. Regardless, taking into account the very definition of the Koszul complex, we know that all the matrices that represent the differentials in $K_{\bullet}\left(y_{1}, \ldots, y_{t}\right)$ have all their entries in $I_{t}$; thus, this single fact implies that all the differentials of the cochain complex $\operatorname{Hom}_{A}\left(K_{\bullet}\left(y_{1}, \ldots, y_{t}\right), A / I_{t}\right)$ vanish and therefore one obtains that

$$
\operatorname{Ext}_{A}^{1}\left(A / I_{t}, A / I_{t}\right)=H^{1}\left(\operatorname{Hom}_{A}\left(K_{\bullet}\left(y_{1}, \ldots, y_{t}\right), A / I_{t}\right)\right)=\left(A / I_{t}\right)^{\oplus t}
$$

In this way, bearing in mind this fact we can arrange the exact sequence (8) in the following way:

$$
0 \longrightarrow A / I_{t+1} \longrightarrow \operatorname{Ext}_{A}^{1}\left(A / I_{t+1}, A / I_{t}\right) \longrightarrow\left(A / I_{t}\right)^{\oplus t} \stackrel{\cdot y_{t+1}}{\longrightarrow}\left(A / I_{t}\right)^{\oplus t}
$$


But the endomorphism on $\left(A / I_{t}\right)^{\oplus t}$ given by multiplication by $Y_{t+1}$ is injective; whence one finally obtains that

$$
A / I_{t+1} \cong \operatorname{Ext}_{A}^{1}\left(A / I_{t+1}, A / I_{t}\right) .
$$

In particular, part (iii) holds.

In addition, we have to point out that part (iv) follows combining part (iii) together with [60, 3.4.14] and Matlis duality in the following way: indeed, we have to notice that

$$
\left(A / I_{t+1}\right)^{\vee}=\operatorname{Ext}_{A}^{1}\left(A / I_{t+1}, A / I_{t}\right)^{\vee} \cong \operatorname{Tor}_{1}^{A}\left(A / I_{t+1},\left(A / I_{t}\right)^{\vee}\right) \cong \operatorname{Tor}_{1}^{A}\left(\left(A / I_{t}\right)^{\vee}, A / I_{t+1}\right)
$$

and therefore $A / I_{t+1} \cong\left(A / I_{t+1}\right)^{\vee \vee} \cong \operatorname{Tor}_{1}^{A}\left(\left(A / I_{t}\right)^{\vee}, A / I_{t+1}\right)^{\vee} \cong \operatorname{Ext}_{A}^{1}\left(\left(0:_{E} I_{t}\right),\left(0:_{E} I_{t+1}\right)\right)$, whence part (iv) also holds.

Finally, since $A / I_{t}$ is a complete intersection ring for any $t$, it is, in particular, quasi Gorenstein. In this way, combining this fact joint with part (iv) one has that

$$
\operatorname{Ext}_{A}^{1}\left(Q_{t}, Q_{t+1}\right) \cong \operatorname{Ext}_{A}^{1}\left(\left(0:_{E} I_{t}\right),\left(0:_{E} I_{t+1}\right)\right)=A / I_{t+1},
$$

just what we finally wanted to show.

\subsubsection{The graded case}

Firstly, we want to review the following notions [32, Definitions 4.1.6 and 4.1.17].

Definition 8.7. Let $\mathbb{K}$ be a field, let $A$ be the polynomial ring $\mathbb{K}\left[x_{1}, \ldots, x_{d}\right]$, and let $m \geq 1$ be an integer.

(i) Given a matrix $W \in \mathcal{M}_{m \times d}(\mathbb{Z})$, we can consider the $\mathbb{Z}^{m}$-grading on $S$ for which $\mathbb{K} \subseteq A_{0}$ and the indeterminates are homogeneous elements whose degrees are given by the columns of $W$. In this case, it is said that $A$ is graded by $W$. Moreover, we refer to the rows of $W$ as the weight vectors of the indeterminates $x_{1}, \ldots, x_{d}$.

(ii) Now, suppose that $A$ is graded by a matrix $W \in \mathcal{M}_{m \times d}(\mathbb{Z})$ of rank $m$ and let $W_{1}, \ldots, w_{m}$ be the weight vectors. It is said that the grading on $A$ given by $W$ is of positive type provided there exist $a_{1}, \ldots, a_{m} \in \mathbb{Z}$ such that all the entries of $a_{1} w_{1}+\ldots+a_{m} w_{m}$ are positive. In this case, it is also said that $W$ is a matrix of positive type. 
Example 8.8. We exhibit some examples of positive-type matrices.

(a) The standard grading on $\mathbb{Z}$ (i.e., $\operatorname{deg}\left(x_{i}\right)=1$ for all $i$ ) is given by matrix $\left(\begin{array}{lll}1 & \ldots & 1\end{array}\right)$, which is clearly of positive type.

(b) The standard $\mathbb{Z}^{d}$-grading on $S$ (i.e., $\operatorname{deg}\left(x_{i}\right)=\mathbf{e}_{i}$, where $\mathbf{e}_{i}$ denotes the element of $\mathbb{Z}^{d}$ that has all its components 0 up to 1 in the $i$ th position) is given by $W=$ the identity matrix of size $d$. It is also clear in this case that $W$ is of positive type; indeed, just take $a_{i}=1$ for all $i$ in the definition.

The reason for which we consider matrices of positive type is the following result, which says that polynomial rings with gradings of positive type and finitely generated graded modules over them have finite-dimensional homogeneous components. We omit its proof and refer to [32, Proposition 4.1.19] for details.

Proposition 8.9. Let $\mathbb{K}$ be a field, let $A$ be the polynomial ring $\mathbb{K}\left[x_{1}, \ldots, x_{d}\right]$ graded by a matrix $W \in \mathcal{M}_{m \times d}(\mathbb{Z})$ of positive type, and let $M$ be a finitely generated $W$-graded $A$-module. Then, the following statements hold:

(a) We have $A_{0}=\mathbb{K}$.

(b) For all $a \in \mathbb{Z}^{m}$, we have $\operatorname{dim}_{\mathbb{K}}\left(M_{a}\right)<+\infty$.

Remark 8.10. It is worth mentioning that the conclusion of the previous proposition also works in greater generality; the interested reader may like to consult [41, Theorem 8.6] for additional details.

In this way, hereafter $\mathbb{K}$ will denote a field and $A$ will stand for the polynomial ring $\mathbb{K}\left[x_{1}, \ldots, x_{d}\right]$ graded by a positive-type matrix $W \in \mathcal{M}_{m \times d}(\mathbb{Z})$. Moreover, let $y_{1}, \ldots, y_{n}$ be homogeneous elements of $A$ (with $\operatorname{deg}\left(Y_{j}\right)=D_{j} \in \mathbb{Z}^{m}$ ) that form an $A$-regular sequence and, for any $1 \leq t \leq n$, set $I_{t}:=\left\langle y_{1}, \ldots, y_{t}\right\rangle$. On the other hand, borrowing notation from $[11,1.5]$ (see also $[8,13.1 .8]) * \operatorname{Hom}_{A}(-,-)$ will stand for the internal Hom in the category of $W$-graded $A$-modules, and set $(-)^{\vee}:={ }^{*} \operatorname{Hom}_{\mathbb{K}}(-, \mathbb{K})$.

The next result gives the $W$-graded analog of [60,3.4.14], which was already used along the proof of Proposition 8.6; albeit its proof is the adaptation in this graded context of [60, Proof of 3.4.14], we provide it for the convenience of the reader.

Proposition 8.11. Let $j \in \mathbb{Z}$ and let $X_{0}$ and $X_{1}$ be $W$-graded $A$-modules. Then, the following statements hold:

(a) $\operatorname{Tor}_{j}^{A}\left(X_{0}, X_{1}\right)^{\vee} \cong{ }^{*} \operatorname{Ext}_{A}^{j}\left(X_{0}, X_{1}^{\vee}\right)$. 
(b) If, in addition, $X_{0}$ is finitely generated, then one has that

$$
\operatorname{Tor}_{j}^{A}\left(X_{0}, X_{1}^{\vee}\right) \cong{ }^{*} \operatorname{Ext}_{A}^{j}\left(X_{0}, X_{1}\right)^{\vee} \text {. }
$$

In any case, both isomorphisms are canonical.

Proof. Firstly, we prove part (a). Indeed, set $T^{j}$ and $U^{j}$ to be the functors $\operatorname{Tor}_{j}^{A}\left(-, X_{1}\right)^{\vee}$ and ${ }^{*} \operatorname{Ext}_{A}^{j}\left(-, X_{1}^{\vee}\right)$. Since $(-)^{\vee}$ is exact and contravariant, it follows that both $\left(T^{j}\right)_{j \in \mathbb{N}}$ and $\left(U^{j}\right)_{j \in \mathbb{N}}$ form a positive strongly connected sequences of contravariant functors. Moreover, it is well known that

$$
{ }^{*} \operatorname{Hom}_{\mathbb{K}}\left(X_{0} \otimes_{A} X_{1}, \mathbb{K}\right) \cong{ }^{*} \operatorname{Hom}_{A}\left(X_{0},{ }^{*} \operatorname{Hom}_{\mathbb{K}}\left(X_{1}, \mathbb{K}\right)\right)
$$

for any $W$-graded $A$-module $X_{0}$; on the other hand, it is also clear that $T^{j} P=0=U^{j} P$ for $j \geq 1$ and any *projective module $P$. Therefore, applying the appropriate dual of $[8,13.3 .5]$ one has that there exist uniquely determined natural equivalences of functors $T^{j} \Longrightarrow U^{j}$; whence part (a) follows directly from this fact.

Finally, we prove part (b). In this case, we set $T^{j}$ and $U^{j}$ as the functors $\operatorname{Tor}_{j}^{A}\left(-, X_{1}^{\vee}\right)$ and ${ }^{*} \operatorname{Ext}_{A}\left(-, X_{1}\right)^{\vee}$. In this case, $\left(T^{j}\right)_{j \in \mathbb{N}}$ and $\left(U^{j}\right)_{j \in \mathbb{N}}$ both form a positive strongly connected sequence of covariant functors. In addition, for a finitely generated $W$-graded $A$-module $X_{0}$ one has a canonical isomorphism

$$
X_{0} \otimes_{A}{ }^{*} \operatorname{Hom}_{\mathbb{K}}\left(X_{1}, \mathbb{K}\right) \cong{ }^{*} \operatorname{Hom}_{\mathbb{K}}\left({ }^{*} \operatorname{Hom}_{A}\left(X_{0}, X_{1}\right), \mathbb{K}\right) .
$$

Again, $T^{j} P=0=U^{j} P$ for $j \geq 1$ and any *projective module $P$. Therefore, applying the appropriate dual of $[8,13.3 .5]$ one has that there exist uniquely determined natural equivalences of functors $T^{j} \Longrightarrow U^{j}$; whence one has that part (b) also holds.

The following statement can be regarded as the $W$-graded analog of Proposition 8.6.

Proposition 8.12. Preserving the foregoing assumptions and notations, the following statements hold:

$$
\begin{aligned}
& \text { (i) }{ }^{*} \operatorname{Hom}_{A}\left(A / I_{t+1}, A / I_{t}\right)=0 . \\
& \text { (ii) }{ }^{*} \operatorname{Hom}_{A}(A, A) \cong A . \\
& \text { (iii) }{ }^{*} \operatorname{Hom}_{A}\left(A / I_{t^{\prime}} A / I_{t}\right) \cong A / I_{t} . \\
& \text { (iv) }{ }^{*} \operatorname{Hom}_{A}\left(A / I_{t^{\prime}}\left(A / I_{t}\right)\left(-D_{t+1}\right)\right) \cong\left(A / I_{t}\right)\left(-D_{t+1}\right) \text {. }
\end{aligned}
$$


(v) ${ }^{*} \operatorname{Ext}_{A}^{1}\left(A / I_{t+1},\left(A / I_{t}\right)\left(-D_{t+1}\right)\right) \cong A / I_{t+1} ;$ more precisely, ${ }^{*} \operatorname{Ext}_{A}^{1}\left(A / I_{t+1},\left(A / I_{t}\right)\right.$ $\left.\left(-D_{t+1}\right)\right)$ is a graded free $\left(A / I_{t+1}\right)$-module of rank 1 that admits as generator the class of the short exact sequence

$$
0 \longrightarrow\left(A / I_{t}\right)\left(-D_{t+1}\right) \stackrel{\cdot t_{t+1}}{\longrightarrow} A / I_{t} \longrightarrow A / I_{t+1} \longrightarrow 0 .
$$

(vi) ${ }^{*} \operatorname{Ext}_{A}^{1}\left(\left(A / I_{t+1}\right)^{\vee},\left(A / I_{t}\right)^{\vee}\right) \cong\left(A / I_{t+1}\right)\left(D_{t+1}\right)$.

(vii) ${ }^{*} \operatorname{Ext}_{A}^{1}\left(Q_{t}, Q_{t+1}\right) \cong\left(A / I_{t+1}\right)\left(D_{t+1}\right)$, where $Q_{t}$ (respectively, $\left.Q_{t+1}\right)$ stands for the local cohomology module $H_{\mathfrak{m}}^{d-t}\left(A / I_{t}\right)$ (respectively, $H_{\mathfrak{m}}^{d-t-1}\left(A / I_{t+1}\right)$ ).

Proof. First of all, we have to point out that, since $A / I_{t}$ is finitely generated, one has that ${ }^{*} \operatorname{Hom}_{A}\left(A / I_{t+1}, A / I_{t}\right)$ is nothing but $\operatorname{Hom}_{A}\left(A / I_{t+1}, A / I_{t}\right)$ in case the grading is forgotten. Regardless, we have checked in part (i) of Proposition 8.6 that $\operatorname{Hom}_{A}\left(A / I_{t+1}, A / I_{t}\right)$ $=0$; whence part (i) follows directly from this fact.

Second, as ${ }^{*} \operatorname{Hom}_{A}(A, A)$ (respectively, ${ }^{*} \operatorname{Hom}_{A}\left(A / I_{t}, A / I_{t}\right)$ ) are nothing but $\operatorname{Hom}_{A}(A, A)$ (respectively, $\operatorname{Hom}_{A}\left(A / I_{t}, A / I_{t}\right)$ ) when the grading is forgotten, we obtain that both parts (ii) and (iii) hold. Moreover, we can also get part (iv) in the below way:

$$
{ }^{*} \operatorname{Hom}_{A}\left(A / I_{t},\left(A / I_{t}\right)\left(-D_{t+1}\right)\right)={ }^{*} \operatorname{Hom}_{A}\left(A / I_{t}, A / I_{t}\right)\left(-D_{t+1}\right)=\left(A / I_{t}\right)\left(-D_{t+1}\right) .
$$

Now, consider the next short exact sequence of $W$-graded $A$-modules and homogeneous homomorphisms:

$$
0 \longrightarrow\left(A / I_{t}\right)\left(-D_{t+1}\right) \stackrel{-Y t+1}{\longrightarrow} A / I_{t} \longrightarrow A / I_{t+1} \longrightarrow 0 .
$$

Applying to such short exact sequence the functor ${ }^{*} \operatorname{Hom}_{A}\left(-,\left(A / I_{t}\right)\left(-D_{t+1}\right)\right)$ one obtains the following exact sequence of $W$-graded $A$-modules and homogeneous homomorphisms:

$$
\begin{aligned}
& 0 \longrightarrow{ }^{*} \operatorname{Hom}_{A}\left(A / I_{t+1},\left(A / I_{t}\right)\left(-D_{t+1}\right)\right) \longrightarrow{ }^{*} \operatorname{Hom}_{A}\left(A / I_{t}\left(A / I_{t}\right)\left(-D_{t+1}\right)\right) \\
& \stackrel{\cdot y t+1}{\longrightarrow}{ }^{*} \operatorname{Hom}_{A}\left(\left(A / I_{t}\right)\left(-D_{t+1}\right),\left(A / I_{t}\right)\left(-D_{t+1}\right)\right) \longrightarrow{ }^{*} \operatorname{Ext}_{A}^{1}\left(A / I_{t+1},\left(A / I_{t}\right)\left(-D_{t+1}\right)\right) \\
& \longrightarrow{ }^{*} \operatorname{Ext}_{A}^{1}\left(A / I_{t^{\prime}}\left(A / I_{t}\right)\left(-D_{t+1}\right)\right) \stackrel{\cdot y_{t+1}}{\longrightarrow}{ }^{*} \operatorname{Ext}_{A}^{1}\left(\left(A / I_{t}\right)\left(-D_{t+1}\right),\left(A / I_{t}\right)\left(-D_{t+1}\right)\right) .
\end{aligned}
$$

We have that ${ }^{*} \operatorname{Hom}_{A}\left(A / I_{t+1},\left(A / I_{t}\right)\left(-D_{t+1}\right)\right)=0,{ }^{*} \operatorname{Hom}_{A}\left(A / I_{t},\left(A / I_{t}\right)\left(-D_{t+1}\right)\right)=$ $\left(A / I_{t}\right)\left(-D_{t+1}\right)$, and ${ }^{*} \operatorname{Hom}_{A}\left(\left(A / I_{t}\right)\left(-D_{t+1}\right),\left(A / I_{t}\right)\left(-D_{t+1}\right)\right)=A / I_{t}$. In this way, we can 
rewrite the previous exact sequence in the next way:

$$
\begin{aligned}
& 0 \longrightarrow\left(A / I_{t}\right)\left(-D_{t+1}\right) \stackrel{\cdot y_{t+1}}{\longrightarrow} A / I_{t} \longrightarrow{ }^{*} \operatorname{Ext}_{A}^{1}\left(A / I_{t+1},\left(A / I_{t}\right)\left(-D_{t+1}\right)\right) \\
& \longrightarrow{ }^{*} \operatorname{Ext}_{A}^{1}\left(A / I_{t},\left(A / I_{t}\right)\left(-D_{t+1}\right)\right) \stackrel{\cdot Y t+1}{\longrightarrow} * \operatorname{Ext}_{A}^{1}\left(\left(A / I_{t}\right)\left(-D_{t+1}\right),\left(A / I_{t}\right)\left(-D_{t+1}\right)\right)
\end{aligned}
$$

Moreover, since the cokernel of $\left(A / I_{t}\right)\left(-D_{t+1}\right) \stackrel{\cdot y_{t+1}}{\longrightarrow} A / I_{t}$ is $A / I_{t+1}$, it follows that we have the following exact sequence:

$$
\begin{aligned}
& 0 \longrightarrow A / I_{t+1} \longrightarrow{ }^{*} \operatorname{Ext}_{A}^{1}\left(A / I_{t+1},\left(A / I_{t}\right)\left(-D_{t+1}\right)\right) \longrightarrow{ }^{*} \operatorname{Ext}_{A}^{1}\left(A / I_{t},\left(A / I_{t}\right)\left(-D_{t+1}\right)\right) \\
& \stackrel{y_{t+1}}{\longrightarrow} \operatorname{Ext}_{A}^{1}\left(\left(A / I_{t}\right)\left(-D_{t+1}\right),\left(A / I_{t}\right)\left(-D_{t+1}\right)\right) .
\end{aligned}
$$

Now, we claim that

$$
{ }^{*} \operatorname{Ext}_{A}^{1}\left(A / I_{t},\left(A / I_{t}\right)\left(-D_{t+1}\right)\right) \cong \bigoplus_{j=1}^{t}\left(A / I_{t}\right)\left(D_{j}-D_{t+1}\right) .
$$

Indeed, it is well known that ${ }^{*} \operatorname{Ext}_{A}^{1}\left(A / I_{t},\left(A / I_{t}\right)\left(-D_{t+1}\right)\right)$ is the 1st cohomology group of the complex ${ }^{*} \operatorname{Hom}_{A}\left(K_{\bullet}\left(y_{1}, \ldots, y_{t}\right),\left(A / I_{t}\right)\left(-D_{t+1}\right)\right)$, where $K_{\bullet}\left(y_{1}, \ldots, y_{t}\right)$ denotes the homological Koszul resolution of $A / I_{t}$. However, since all the spots in ${ }^{*} \operatorname{Hom}_{A}\left(K_{\bullet}\left(y_{1}, \ldots, y_{t}\right)\right.$, $\left.\left(A / I_{t}\right)\left(-D_{t+1}\right)\right)$ are $A / I_{t}$-modules and all the differentials $\partial^{i}$ in such cochain complex are represented by matrices with entries in $I_{t}$, it follows that all these differentials are zero and therefore

$$
{ }^{*} \operatorname{Ext}_{A}^{1}\left(A / I_{t},\left(A / I_{t}\right)\left(-D_{t+1}\right)\right)=\operatorname{ker}\left(\partial^{1}\right)=\bigoplus_{j=1}^{t}\left(A / I_{t}\right)\left(D_{j}-D_{t+1}\right)
$$

In addition, by similar reasons one also has that

$$
{ }^{*} \operatorname{Ext}_{A}^{1}\left(\left(A / I_{t}\right)\left(-D_{t+1}\right),\left(A / I_{t}\right)\left(-D_{t+1}\right)\right) \cong \bigoplus_{j=1}^{t}\left(A / I_{t}\right)\left(D_{j}\right)
$$

In this way, the map

$$
{ }^{*} \operatorname{Ext}_{A}^{1}\left(A / I_{t},\left(A / I_{t}\right)\left(-D_{t+1}\right)\right) \stackrel{\cdot y_{t+1}}{\longrightarrow}{ }^{*} \operatorname{Ext}_{A}^{1}\left(\left(A / I_{t}\right)\left(-D_{t+1}\right),\left(A / I_{t}\right)\left(-D_{t+1}\right)\right)
$$


can be rewritten in the following way:

$$
\bigoplus_{j=1}^{t}\left(A / I_{t}\right)\left(D_{j}-D_{t+1}\right) \quad \stackrel{y^{t+1}}{\longrightarrow} \bigoplus_{j=1}^{t}\left(A / I_{t}\right)\left(D_{j}\right)
$$

But this homomorphism is clearly injective. In this way, combining this fact joint with (2) one finally obtains that

$$
A / I_{t+1} \cong{ }^{*} \operatorname{Ext}_{A}^{1}\left(A / I_{t+1},\left(A / I_{t}\right)\left(-D_{t+1}\right)\right)={ }^{*} \operatorname{Ext}_{A}^{1}\left(A / I_{t+1}, A / I_{t}\right)\left(-D_{t+1}\right),
$$

whence part $(\mathrm{v})$ holds too. The reader will easily note that the righmost equality is well known [8, 14.1.10].

Now, we can deduce part (vi) combining part (v) jointly with Proposition 8.11 in the following manner:

$$
\begin{aligned}
A / I_{t+1} & \cong\left(\left(A / I_{t+1}\right)^{\vee}\right)^{\vee} \cong\left({ }^{*} \operatorname{Ext}_{A}^{1}\left(A / I_{t+1},\left(A / I_{t}\right)\left(-D_{t+1}\right)\right)^{\vee}\right)^{\vee} \\
& \cong \operatorname{Tor}_{1}^{A}\left(A / I_{t+1},\left[\left(A / I_{t}\right)\left(-D_{t+1}\right)\right]^{\vee}\right)^{\vee} \cong \operatorname{Tor}_{1}^{A}\left(\left(A / I_{t}\right)^{\vee}\left(D_{t+1}\right), A / I_{t+1}\right)^{\vee} \\
& \cong{ }^{*} \operatorname{Ext}_{A}^{1}\left(\left(A / I_{t}\right)^{\vee}\left(D_{t+1}\right),\left(A / I_{t+1}\right)^{\vee}\right) .
\end{aligned}
$$

Finally, the graded local duality theorem $[8,14.4 .1]$ implies that $\left(A / I_{t}\right)^{\vee}(-C) \cong Q_{t}$ and $\left(A / I_{t+1}\right)^{\vee}(-c) \cong Q_{t+1}$, where $c=c_{1}+\ldots+c_{d}$ and $c_{1}, \ldots, c_{d}$ are the columns of matrix $W$. Whence

$$
{ }^{*} \operatorname{Ext}_{A}^{1}\left(Q_{t}, Q_{t+1}\right) \cong{ }^{*} \operatorname{Ext}_{A}^{1}\left(\left(A / I_{t}\right)^{\vee}(-C),\left(A / I_{t+1}\right)^{\vee}(-C)\right) \cong{ }^{*} \operatorname{Ext}_{A}^{1}\left(\left(A / I_{t}\right)^{\vee},\left(A / I_{t+1}\right)^{\vee}\right)
$$

But the leftmost term is isomorphic to $\left(A / I_{t+1}\right)\left(D_{t+1}\right)$; the proof is therefore completed.

\subsection{A Gräbe's type description formula}

The results obtained in Proposition 8.12 can be regarded as a Gräbe's type description formula (cf. [22, Theorem 2]) because they describe the $W$-graded structure of these local cohomology modules by looking at these groups of extensions; more precisely, we have the following:

Theorem 8.13. Let $\mathbb{K}$ be a field, and let $A$ be the polynomial ring $\mathbb{K}\left[x_{1}, \ldots, x_{d}\right]$ graded by a positive-type matrix $W \in \mathcal{M}_{m \times d}(\mathbb{Z})$. Moreover, for any $1 \leq t \leq d$ set $F_{t}$ as the 
face ideal of $A$ generated by $x_{1}, \ldots, x_{t}$ and $Q_{t}:=H_{\mathfrak{m}}^{d-t}\left(A / F_{t}\right)$. Then, there is a canonical isomorphism

$$
{ }^{*} \operatorname{Ext}_{A}^{1}\left(Q_{t}\left(c_{t+1}\right), Q_{t+1}\right) \cong\left(A / F_{t+1}\right),
$$

where $c_{t+1}$ denotes the $(t+1)$ th column of matrix $W$; more precisely, ${ }^{*} \operatorname{Ext}_{A}^{1}\left(Q_{t}\left(c_{t+1}\right), Q_{t+1}\right)$ is a free, graded $\left(A / F_{t+1}\right)$-module that admits as generator the class of the short exact sequence

$$
0 \longrightarrow Q_{t+1} \longrightarrow Q_{t} \stackrel{\cdot x_{t+1}}{\longrightarrow} Q_{t}\left(c_{t+1}\right) \longrightarrow 0
$$

Remark 8.14. When $W$ is the identity matrix of size $d$, Theorem 8.13 describes the standard $\mathbb{Z}^{d}$-graded structure of local cohomology modules of Stanley-Reisner rings; in [22, Theorem 2]; Gräbe gave a nice topological interpretation of this structure by identifying the multiplication by each variable on the graded pieces of local cohomology modules in terms of certain connecting maps between simplicial cohomology groups. In this way, by means of Gräbe's formula one can also describe these groups of extensions in terms of the corresponding simplicial complex. On the other hand, Miller proved in his thesis [40, Corollary 6.24] that Gräbe's formula is equivalent to the one obtained by Mustaţă in [42, Theorem 2.1 and Corollary 2.2]; keeping in mind this fact, Theorem 8.13 can also be regarded as a Mustaţă-type description formula.

\section{Funding}

This work was supported by Generalitat de Catalunya [2017SGR-932 to J.A.M., 2017SGR-585 to S.Z.]; Spanish Ministerio de Economía y Competitividad [MTM2015-69135-P to J.A.M., MTM20167881-P to A.F.B., MTM2016-7881-P to S.Z.]; and Israel Science Foundation [844/14 to A.F.B.].

\section{Acknowledgments}

The authors also would like to thank Jack Jeffries, Wenliang Zhang, and the anonymous referee for their comments on an earlier draft of this manuscript. Part of this work was done when A.F.B. visited Northwestern University funded by the CASB fellowship program. Dedicated to the memory of Jan-Erik Roos (1935-2017).

\section{References}

[1] Alba-Sarria, L., R. Callejas-Bedregal, and N. Caro-Tuesta. "Finiteness properties of local cohomology modules over differentiable admissible algebras." J. Pure Appl. Algebra 221, no. 9 (2017): 2236-49.

[2] Alberelli, D. "Local Picard groups of binoids and their algebras". PhD thesis, Universität Osnabrück , 2016. https://arxiv.org/pdf/1611.01788.pdf. 
[3] Alberelli, D. and H. Brenner. "Local Picard group of pointed monoids and their algebras." Preprint https://arxiv.org/pdf/1802.02793.pdf.

[4] Àlvarez Montaner, J. and A. Vahidi. "Lyubeznik numbers of monomial ideals." Trans. Amer. Math. Soc. 366, no. 4 (2014): 1829-55.

[5] Àlvarez Montaner, J., R. García López, and S. Zarzuela Armengou. "Local cohomology, arrangements of subspaces and monomial ideals." Adv. Math. 174, no. 1 (2003): 35-56.

[6] Bijan-Zadeh, M. H. "A common generalization of local cohomology theories." Glasgow Math. J. 21, no. 2 (1980): 173-181.

[7] Björner, A. and T. Ekedahl. "Subspace arrangements over finite fields: cohomological and enumerative aspects." Adv. Math. 129, no. 2 (1997): 159-87.

[8] Brodmann, M. P. and R. Y. Sharp. Local Cohomology: An Algebraic Introduction with Geometric Applications, 2nd ed. Cambridge Studies in Advanced Mathematics 136. Cambridge: Cambridge University Press, 2013.

[9] Brun, M. and T. Römer. “On algebras associated to partially ordered sets." Math. Scand. 103, no. 2 (2008): 169-185.

[10] Brun, M., W. Bruns, and T. Römer. "Cohomology of partially ordered sets and local cohomology of section rings." Adv. Math. 208, no. 1 (2007): 210-35.

[11] Bruns, W. and J. Herzog. Cohen-Macaulay Rings, revised ed. Cambridge Studies in Advanced Mathematics 39. Cambridge: Cambridge University Press, 1998.

[12] Bruns, W., R. Koch, and T. Römer. "Gröbner bases and Betti numbers of monoidal complexes." Michigan Math. J. 57 (2008): 71-91. Special volume in honor of Melvin Hochster.

[13] Castaño Domínguez, A. “Two Mayer-Vietoris spectral sequences for D-modules." In Trends in Number Theory, 17-28. Contemporary Mathematics 649. Providence, RI: American Mathematical Society, 2015.

[14] Coutinho, S. C. A Primer of Algebraic D-modules. London Mathematical Society Student Texts 33. Cambridge: Cambridge University Press, 1995.

[15] Derksen, H. and J. Sidman. "A sharp bound for the Castelnuovo-Mumford regularity of subspace arrangements." Adv. Math. 172, no. 2 (2002): 151-7.

[16] Divaani-Aazar, K. and R. Sazeedeh. "Cofiniteness of generalized local cohomology modules." Colloq. Math. 99, no. 2 (2004): 283-90.

[17] Eisenbud, D. The Geometry of Syzygies: A Second Course in Commutative Algebra and Algebraic Geometry. Graduate Texts in Mathematics 229. New York: Springer, 2005.

[18] Eisenbud, D., M. Mustaţă, and M. Stillman. "Cohomology on toric varieties and local cohomology with monomial supports." J. Symbolic Comput. 29, nos. 4-5 (2000): 583-600. Symbolic computation in algebra, analysis, and geometry (Berkeley, CA, 1998).

[19] Enescu, F. and M. Hochster. "The Frobenius structure of local cohomology." Algebra Number Theory 2, no. 7 (2008): 721-54.

[20] Goresky, M. and R. MacPherson. Stratified Morse Theory. Ergebnisse der Mathematik und ihrer Grenzgebiete (3) 14. Berlin: Springer, 1988.

[21] Goto, S. and K.-I. Watanabe. “On graded rings. I." J. Math. Soc. Japan 30, no. 2 (1978): 179-213. 
[22] Gräbe, H.-G. "The canonical module of a Stanley-Reisner ring." J. Algebra 86, no. 1 (1984): 272-81.

[23] Grayson, D. R. and M. E. Stillman. Macaulay2, a software system for research in algebraic geometry, 2013. http://www.math.uiuc.edu/Macaulay2/.

[24] Heinzer, W., A. Mirbagheri, L. J. Ratliff, Jr., and K. Shah. "Parametric decomposition of monomial ideals. II." J. Algebra 187, no. 1 (1997): 120-49.

[25] Hellus, M. and P. Schenzel. “On cohomologically complete intersections.” J. Algebra 320, no. 10 (2008): 3733-48.

[26] Herzog, J. and T. Hibi. Monomial Ideals. Graduate Texts in Mathematics 260. London: Springer London, Ltd., 2011.

[27] Hochster, M. and C. Huneke. "Tight closure, invariant theory, and the Briançon-Skoda theorem." J. Amer. Math. Soc. 3, no. 1 (1990): 31-116.

[28] Ichim, B. and T. Römer. “On toric face rings." J. Pure Appl. Algebra 210, no. 1 (2007): 249-66.

[29] Iyengar, S. B., G. J. Leuschke, A. Leykin, C. Miller, E. Miller, A. K., Singh, and U. Walther. Twenty-four Hours of Local Cohomology. Graduate Studies in Mathematics 87. Providence, RI: American Mathematical Society, 2007.

[30] Jensen, C. U. Les Foncteurs Dérivés de lim et leurs Applications en Théorie des Modules. Lecture Notes in Mathematics 254. Berlin-New York: Springer, 1972.

[31] Katzman, M., L. Ma, I. Smirnov, and W. Zhang. "D-module and F-module length of some local cohomology modules." Trans. Amer. Math. Soc. https://doi.org/10.1090/tran/7266.

[32] Kreuzer, M. and L. Robbiano. Computational Commutative Algebra 2. Berlin: Springer, 2005.

[33] Laudal, O. A. "Sur les limites projectives et inductives." Ann. Sci. École Norm. Sup. (3)82 (1965): 241-96.

[34] Lyubeznik, G. "On the local cohomology modules $H_{\mathfrak{a}}^{i}(R)$ for ideals a generated by monomials in an $R$-sequence." In Complete Intersections (Acireale, 1983), 214-20. Lecture Notes in Math 1092. Berlin: Springer, 1984.

[35] Lyubeznik, G. "Finiteness properties of local cohomology modules (an application of $D$ modules to commutative algebra)." Invent. Math. 113, no. 1 (1993): 41-55.

[36] Lyubeznik, G. " $F$-modules: applications to local cohomology and $D$-modules in characteristic p > 0." J. Reine Angew. Math. 491 (1997): 65-130.

[37] Lyubeznik, G. "On some local cohomology modules." Adv. Math. 213, no. 2 (2007): 621-43.

[38] Maclagan, D. and G. G. Smith. "Multigraded Castelnuovo-Mumford regularity." J. Reine Angew. Math. 571 (2004): 179-212.

[39] Miller, E. “Resolutions and duality for monomial ideals." PhD thesis, University of California, Berkeley, 2000. ProQuest LLC, Ann Arbor, MI, 2000.

[40] Miller, E. "The Alexander duality functors and local duality with monomial support." $J$. Algebra 231, no. 1 (2000): 180-234.

[41] Miller, E. and B. Sturmfels. Combinatorial Commutative Algebra. Graduate Texts in Mathematics 227. New York: Springer, 2005.

[42] Mustaţă, M. "Local cohomology at monomial ideals." J. Symbolic Comput. 29, nos. 4-5 (2000): 709-20. 
[43] Narváez-Macarro, L. Differential Structures in Commutative Algebra. Mini-course at the XXIII Brazilian Algebra Meeting, July 27-August 1. Brazil: Maringá, 2014. http://personal. us.es/narvaez/DSCA_course_2014.pdf.

[44] Năstăsescu, C. "Some constructions over graded rings: applications." J. Algebra 120, no. 1 (1989): 119-38.

[45] Neeman, A. Triangulated Categories. Annals of Mathematics Studies 148. Princeton, N.J.: Princeton University Press, 2001.

[46] Nguyen, D. H. "Seminormality and local cohomology of toric face rings." J. Algebra 371 (2012): $536-53$.

[47] Nöbeling, G. "Über die derivierten des inversen und des direkten limes einer Modulfamilie." Topology 1 (1962): 47-61.

[48] Núñez Betancourt, L. “On certain rings of differentiable type and finiteness properties of local cohomology." J. Algebra 379 (2013): 1-10.

[49] Núñez-Betancourt, L. and E. E. Witt. "Generalized Lyubeznik numbers." Nagoya Math. J. 215 (2014): 169-201.

[50] Okazaki, R. and K. Yanagawa. "Dualizing complex of a toric face ring." Nagoya Math. J. 196 (2009): 87-116.

[51] Peskine, C. and L. Szpiro. "Dimension projective finie et cohomologie locale. Applications à la démonstration de conjectures de M. Auslander, H. Bass et A. Grothendieck." Inst. Hautes Études Sci. Publ. Math. 42 (1973): 47-119.

[52] Puthenpurakal, T. J. "Injective modules over some rings of differential operators." (2013): preprint http://arxiv.org/pdf/1301.1176v1.

[53] Rahimi, A. "Tameness of local cohomology of monomial ideals with respect to monomial prime ideals." J. Pure Appl. Algebra 211, no. 1 (2007): 83-93.

[54] Raicu, C. "Regularity and cohomology of determinantal thickenings." Proc. London Math. Soc. 116, no. 2 (2018): 248-80.

[55] Roos, J.-E. "Sur les foncteurs dérivés de lim. Applications." C. R. Acad. Sci. Paris 252 (1961): 3702-3704.

[56] Rotman, J. J. An Introduction to Homological Algebra, 2nd ed. Universitext. New York: Springer, 2009.

[57] Sabzrou, H. and M. Tousi. "Local cohomology at monomial ideals in R-sequences." Comm. Algebra 36, no. 1 (2008): 37-52.

[58] Smith, K. E. "F-rational rings have rational singularities." Amer. J. Math. 119, no. 1 (1997): 159-80.

[59] Stanley, R. P. Combinatorics and Commutative Algebra, 2nd ed. Progress in Mathematics 41. Boston, MA: Birkhäuser Boston Inc., 1996.

[60] Strooker, J. R. Homological Questions in Local Algebra. London Mathematical Society Lecture Note Series 145. Cambridge: Cambridge University Press, 1990.

[61] Takahashi R., Y. Yoshino, and T. Yoshizawa. "Local cohomology based on a nonclosed support defined by a pair of ideals." J. Pure Appl. Algebra 213, no. 4 (2009): 582-600. 
[62] Takayama, Y. “Combinatorial characterizations of generalized Cohen-Macaulay monomial ideals." Bull. Math. Soc. Sci. Math. Roumanie (N.S.) 48(96), no. 3 (2005): 327-44.

[63] Terai, N. "Local cohomology modules with respect to monomial ideals." 1999. Unpublished preprint.

[64] Yanagawa, K. "Bass numbers of local cohomology modules with supports in monomial ideals." Math. Proc. Cambridge Philos. Soc. 131, no. 1 (2001): 45-60.

[65] Yanagawa, K. "Sheaves on finite posets and modules over normal semigroup rings." J. Pure Appl. Algebra 161, no. 3 (2001): 341-66.

[66] Yanagawa, K. "Notes on $C$-graded modules over an affine semigroup ring $K[C]$." Comm. Algebra 36, no. 8 (2008): 3122-46.

[67] Yassemi, S. “Generalized section functors." J. Pure Appl. Algebra 95, no. 1 (1994): 103-19. 